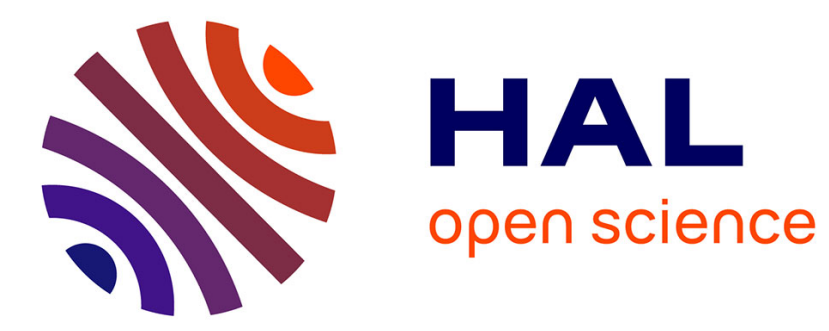

\title{
Direct numerical simulations of supersonic turbulent channel flows of dense gases
}

Luca Sciacovelli, Paola Cinnella, Xavier Gloerfelt

\section{To cite this version:}

Luca Sciacovelli, Paola Cinnella, Xavier Gloerfelt. Direct numerical simulations of supersonic turbulent channel flows of dense gases. Journal of Fluid Mechanics, 2017, 821, pp.153-199. 10.1017/jfm.2017.237 . hal-02173507

\section{HAL Id: hal-02173507 https://hal.science/hal-02173507}

Submitted on 4 Jul 2019

HAL is a multi-disciplinary open access archive for the deposit and dissemination of scientific research documents, whether they are published or not. The documents may come from teaching and research institutions in France or abroad, or from public or private research centers.
L'archive ouverte pluridisciplinaire HAL, est destinée au dépôt et à la diffusion de documents scientifiques de niveau recherche, publiés ou non, émanant des établissements d'enseignement et de recherche français ou étrangers, des laboratoires publics ou privés. 


\title{
Direct numerical simulations of supersonic turbulent channel flows of dense gases
}

\author{
L. Sciacovelli ${ }^{1,2}$, P. Cinnella ${ }^{1, \dagger}$ and X. Gloerfelt ${ }^{1}$ \\ ${ }^{1}$ Laboratoire DynFluid, Arts et Métiers ParisTech, 75013 Paris, FR, France \\ ${ }^{2}$ Dipartimento di Meccanica, Matematica e Management, Politecnico di Bari, 70125 Bari, IT, Italy
}

The influence of dense-gas effects on compressible wall-bounded turbulence is investigated by means of direct numerical simulations of supersonic turbulent channel flows. Results are obtained for PP11, a heavy fluorocarbon representative of dense gases, the thermophysics properties of which are described by using a fifth-order virial equation of state and advanced models for the transport properties. In the dense-gas regime, the speed of sound varies non-monotonically in small perturbations and the dependency of the transport properties on the fluid density (in addition to the temperature) is no longer negligible. A parametric study is carried out by varying the bulk Mach and Reynolds numbers, and results are compared to those obtained for a perfect gas, namely air. Dense-gas flow exhibits almost negligible friction heating effects, since the high specific heat of the fluids leads to a loose coupling between thermal and kinetic fields, even at high Mach numbers. Despite negligible temperature variations across the channel, the mean viscosity tends to decrease from the channel walls to the centreline (liquid-like behaviour), due to its complex dependency on fluid density. On the other hand, strong density fluctuations are present, but due to the non-standard sound speed variation (opposite to the mean density evolution across the channel), the amplitude is maximal close to the channel wall, i.e. in the viscous sublayer instead of the buffer layer like in perfect gases. As a consequence, these fluctuations do not alter the turbulence structure significantly, and Morkovin's hypothesis is well respected at any Mach number considered in the study. The preceding features make high Mach wall-bounded flows of dense gases similar to incompressible flows with variable properties, despite the significant fluctuations of density and speed of sound. Indeed, the semi-local scaling of Patel et al. (Phys. Fluids, vol. 27 (9), 2015, 095101) or Trettel \& Larsson (Phys. Fluids, vol. 28 (2), 2016, 026102) is shown to be well adapted to compare results from existing surveys and with the well-documented incompressible limit. Additionally, for a dense gas the isothermal channel flow is also almost adiabatic, and the Van Driest transformation also performs reasonably well. The present observations open the way to the development of suitable models for dense-gas turbulent flows.

Key words: compressible turbulence, turbulence simulation, turbulent flows

$\dagger$ Email address for correspondence: paola.cinnella@ensam.eu 


\section{Introduction}

Turbulent flows of dense gases represent a research field of great importance for a wide range of applications in the fields of process engineering and energy conversion cycles. Dense gases are usually defined as single-phase fluids with complex heavy molecules, which operate in the vapour phase at pressures and temperatures of the order of magnitude of those of the liquid/vapour critical point. The dynamics of dense-gas flows is governed by the fundamental derivative of gas dynamics (Thompson 1971):

$$
\Gamma=1+\frac{\rho}{c}\left(\frac{\partial c}{\partial \rho}\right)_{s},
$$

where $\rho$ is the fluid density, $c$ is the speed of sound and $s$ is the entropy, which measures relative variations of the speed of sound in isentropic transformations (Cramer \& Kluwick 1984) and is directly related to the molecular complexity of the gas (Guardone \& Argrow 2005). It is usual to identify dense gases as fluids that exhibit values of $\Gamma$ lower than one in their vapour phase. In such conditions, the speed of sound tends to decrease in isentropic compressions and increase in expansions, contrary to the behaviour of lighter, 'classical' gases, like air or steam. The reversed variations of the speed of sound deeply modify the behaviour of dense-gas flows in the transonic and supersonic regime compared to more standard fluids. The most striking differences are expected for the so-called Bethe-Zel'dovich-Thompson (BZT) fluids, which exhibit a region of negative $\Gamma$ values in their vapour phase (inversion zone), leading to the appearance of non-classical phenomena like expansion shocks in some ranges of thermodynamic conditions (Cramer \& Kluwick 1984). More details about the peculiar gas dynamics of dense gases and more specifically of BZT fluids can be found in Cinnella \& Congedo (2007).

Despite the increasing interest in using dense gases in applications, such as organic Rankine cycles (ORC) (see, e.g. Monaco, Cramer \& Watson 1997; Brown \& Argrow 2000; Congedo, Corre \& Cinnella 2011), the influence of dense-gas effects on turbulence remains unexplored because experimental measurements are very difficult. In particular, understanding and modelling high-speed flow effects is of utmost importance for the design and performance analysis of supersonic ORC turbine expanders (Bufi \& Cinnella 2015). The dynamics of compressible turbulence in the presence of strong dense-gas effects was first investigated in Sciacovelli et al. (2016a). In this work, Euler-based numerical simulations were used to study the large-scale dynamics of compressible homogeneous isotropic turbulence (HIT) of a BZT gas, for which expansion shocks may occur at particular thermodynamic conditions in the transonic and supersonic flow regions. At sufficiently high turbulent Mach number, compressible HIT is significantly modified by dense-gas effects. Specifically, it was observed that the reversed speed of sound behaviour leads to large density and speed of sound fluctuations despite negligible temperature fluctuations, and to much more symmetric probability distribution functions, e.g. of the local dilatation, due to the weakening of compressive structures and the enhancement of expansion ones. For the dense-gas, strong expansion regions are characterized by sheet-like structures, unlike the perfect gas which exhibits tubular structures. Moreover, expansion eddy shocklets may appear in the dense gas. Additionally, viscous studies were carried out to elucidate dense-gas effects on the small-scale dynamics (Sciacovelli \& Cinnella 2015; Sciacovelli, Cinnella \& Grasso 2016b). Strong expansion regions are found to contribute to the dilatational dissipation by an amount comparable to compression regions. This is due to the occurrence of steep expanding structures and possibly 
of expansion shocklets which contribute to enstrophy generation and counterbalance enstrophy destruction introduced by eddy-like structures.

Wall turbulence includes several phenomena that are absent in HIT, such as the mean shear or variations of mean density. A peculiar feature in the dense-gas regime is that the dynamic viscosity $\mu$ and the thermal conductivity $\kappa$ depend both on temperature and pressure through complex relationships. Consequently, a dense gas can exhibit both gas-like behaviour, with strong compressibility effects due to the non-standard variations of its sound speed, and liquid-like features with a viscosity decreasing as the temperature increases. We also stress here that, differently from perfect gases, the transport properties of dense gases also depend on density (or pressure) and not on temperature only. This dependency also represents a major difference with respect to variable-property liquids, whose density and viscosity depend on the temperature only. Note that the heat capacity $c_{p}$ of a dense gas is generally much larger than the one of a classical gas, implying a weaker coupling between the dynamic and thermal boundary layers, so that isothermal transformations are also almost adiabatic. Even for supersonic flows, characterized by high values of the Mach number, friction heating is expected to be reduced, leading to small temperature variations, like in the incompressible flow limit. The overall behaviour is nonetheless unforeseen due for instance to strong variations of Prandtl number $\operatorname{Pr}$ in regions where $c_{p}$ becomes large. In summary, the peculiar variations of the thermodynamic and transport properties in dense gases are expected to alter the conventional behaviour of turbulence via complex nonlinear couplings.

The coupling between turbulence and state variables is a problem of fundamental interest and has been discussed notably for high-speed flows, where compressibility effects come into play, or in the context of strong variations of properties where the temperature can no longer be considered as a passive scalar.

The question of the effects of compressibility on the structure of wall-bounded turbulence is tightly related to variations of the density, temperature, viscosity and thermal conductivity, leading to variations of the Reynolds and Mach numbers across the flow. Chu \& Kovasznay (1958) proposed a general decomposition into vorticity, acoustic and entropic modes and analysed systematically their interactions up to the second order. The effects of variable transport properties on heat transfer or large-scale coherent structures can then be viewed as interactions between vorticity and entropy modes. For supersonic wall-bounded flows, the main lesson learned from previous studies is that the vortical motions are weakly affected by variations of mean properties and the large-scale organization of wall turbulence remains close to the one that has been extensively described for incompressible wall-bounded turbulence (Spina, Smits \& Robinson 1994). The nonlinear coupling with acoustic or entropy modes (Chu \& Kovasznay 1958) does not generate substantial sources of the vorticity mode. This passive character of compressible wall turbulence is well summarized by Morkovin's hypothesis (Morkovin 1961), i.e. that compressibility mainly influences the turbulence through variations in mean density, whereas the density fluctuations have a negligible effect. Compressibility mainly modulates the turbulence through variations in the mean values of thermodynamic quantities. Since the flow dynamics essentially follows an incompressible pattern, a lot of studies have focused on the incompressible-compressible correspondence. Indeed, since no universal theory for wall-bounded compressible flows is available so far, the incompressible limit remains an interesting reference to quantify the alteration of turbulence structure. In particular, efforts have been made to extend conventional scaling laws for constant property flows to compressible conditions (Bradshaw 1977). 
Understanding how the peculiar variations of the thermodynamic and transport properties typical of dense gases influence the behaviour of wall-bounded turbulent flows is of primal importance for providing a correct description of friction drag and heat fluxes in many applications, and to develop suitable models for predicting them. Measurements of dense-gas flows are hardly available due to the difficulty of conducting measurements of this kind of flows. Furthermore, most of the facilities presently under development are thought to provide average pressure and temperature profiles and, in some cases, flow visualizations, but are not expected to provide a detailed description of the turbulence structure, at least in the short term. A review of recent experimental research on dense-gas flows can be found in Spinelli et al. (2013) and Mathijssen et al. (2015). For these reasons, in this work we choose to investigate wall-bounded turbulent dense-gas flows by means of direct numerical simulations (DNS) of the Navier-Stokes equations, supplemented by suitable thermodynamic and transport property models. More specifically, we conduct parametric numerical experiments for the compressible turbulent channel flow. This configuration is well suited for a systematic study of wall-bounded turbulence without complicating features such as streamwise development, shocks or separation.

The first DNS of compressible turbulent channel flow of a perfect gas have been performed by Coleman, Kim \& Moser (1995) and Huang, Coleman \& Bradshaw (1995). They considered a plane channel flow between two (cooled) isothermal walls for two values of the bulk Mach number, namely 1.5 and 3, and two values of the Reynolds number based on bulk quantities and wall viscosity (3000 and 4880, respectively). They found that the flow is influenced by the strong wall-normal gradients of the mean density. One of the main results was the confirmation of the validity of the Morkovin hypothesis. Data were further analysed by Huang et al. (1995) who introduced a semi-local scaling where local thermodynamic properties for density and viscosity replaces wall values to collapse turbulent statistics. The agreement with incompressible data is improved for mean streamwise velocity profiles compared to the Van Driest transformation (Van Driest 1951) often used for turbulent boundary layers with adiabatic walls. Lechner, Sesterhenn \& Friedrich (2001) reproduced the DNS of Coleman et al. (1995) at $M=1.5$ and $R e=3000$ and studied scatter plots to analyse sweeps and ejections in the wall layer. They reported a slight change in Reynolds stresses anisotropy compared to the incompressible case. Foysi, Sarkar \& Friedrich (2004) investigated turbulent channel flow with isothermal walls using DNS with Mach numbers up to 3.5 and Reynolds numbers based on friction velocity $\left(R e_{\tau}\right)$ up to 1030 . They focused on the behaviour of pressure fluctuations and also found that turbulent stresses are mainly affected by mean property variation so that the semi-local scaling is well suited for comparison with incompressible cases. Morinishi, Tamano \& Nakabayashi (2004) complemented the study of Coleman et al. (1995) by investigating the influence of the thermal condition at the wall. They performed DNS of compressible channel flows between both adiabatic and isothermal walls at Mach number 1.5. Tamano \& Morinishi (2006) considered the asymmetric case of a channel with a cold and a hot wall, either adiabatic or isothermal. Heat transfers can reduce compressibility effects but remain of limited importance, and the Morkovin hypothesis is still applicable. Brun et al. (2008) performed large-eddy simulations of fully developed channel flows with specific focus on wall modelling. They proposed a variant of the semi-local scaling for both amplitude and distance and arrive at the same conclusions as Huang et al. (1995) or Morinishi et al. (2004). Wei \& Pollard (2011) studied the influence of the Mach number, $M=0.2,0.7$ and 1.5, at constant bulk Reynolds number and 
proposed budgets of transport properties. Gerolymos \& Vallet (2014) performed a series of DNS and derived transport equations to study in detail the variance of thermodynamic fluctuations. Trettel \& Larsson (2016) gave a theoretical framework to the semi-local scaling of Huang et al. (1995) showing that the scaling of the wall distance is important to take into account the variable mean properties. A good match with incompressible distributions is found for a wide range of Reynolds and Mach numbers. Recently, Modesti \& Pirozzoli (2016) produced an extensive DNS database increasing the bulk Reynolds number up to $R e=34000$. They applied the different scalings proposed in previous studies and showed that the transformation of Trettel \& Larsson (2016) very well reproduces the behaviour of the mean velocity profile by matching the friction Reynolds number based on semi-local quantities.

In terms of coherent motions in the wall layer, Coleman et al. (1995) identified an enhanced coherence of near-wall streaks when compared to the incompressible references. Greater streak elongation was also reported for supersonic boundary layers with cooled walls, whereas a tendency towards shorter streaks is noted for heated walls (Duan, Beekman \& Martin 2010; Lagha et al. 2011). Morinishi et al. (2004) noted that streaks do not become more coherent if the semi-local scaling is used instead of wall units to non-dimensionalize the lengths. This was confirmed later by Patel et al. (2015) for low Mach number flows with variable properties.

Given the importance of mean density and viscosity variations, other studies have considered turbulent channel flow with variable properties without compressibility effects due to high speed. Some studies have investigated heat transfers in turbulent channel flow by treating temperature as a passive scalar. For instance, Teitel \& Antonia (1993) considered the influence of different thermal wall conditions and noted the importance of the value of the Prandtl number for heat transfers. Nicoud \& Poinsot (1999) investigated by DNS the effects of variable density and molecular viscosity on a channel flow between two isothermal walls for different temperature ratios or viscosity laws. Low Mach simulations are used and support Van Driest scaling for mean velocity profiles and semi-local scaling for Reynolds stresses. Several works have investigated the effects of temperature-dependent properties using a nonlinear coupling algorithm (Bae, Yoo \& Choi 2005; Li et al. 2008; Sewall \& Tafti 2008; Zonta, Marchioli \& Soldati 2012). In all of these studies the viscosity, density and thermal conductivity vary with the temperature. Zonta et al. (2012) considered temperature gradients in water channel flow and examined the turbulent kinetic energy budgets. Lee et al. (2013) showed that the viscosity stratification and associated wall heating can alter the ejections and sweeps events in an incompressible turbulent boundary layer. Bae et al. (2005) studied the turbulent heat transfer mechanisms of supercritical carbon dioxide $\left(\mathrm{CO}_{2}\right)$ performing DNS in heated vertical tubes. They focused on the well-known heat transfer deterioration phenomenon in supercritical flows. Li et al. (2008) investigated the same fluid in a long plane channel by imposing wall temperature variations in the streamwise direction, i.e. considering heating and cooling regions at the beginning and at the end of the channel, respectively. They found that the effects of density fluctuations, particularly near the pseudo-critical temperature, on turbulent kinetic energy cannot be ignored, and that the different temperature values cause a modulation of the near-wall turbulent structures. Patel et al. (2015) conducted numerical experiments by imposing different distributions for the fluid properties to investigate separately the effects of mean density or viscosity gradients in low Mach number turbulent channel flow. They showed that various laws bearing similarities either with liquid-like or gas-like behaviour can be taken into account for turbulent statistics by using the semi-local scaling introduced in the 
compressible regime. In particular, a good similarity was observed for an equivalent friction Reynolds number constructed with semi-local quantities.

To the authors' knowledge, compressible wall-bounded flows have been almost exclusively investigated for air, modelled as a perfect polytropic gas. A few studies have considered variable-property or supercritical fluids with a low Mach number assumption. In this work, we present new direct numerical simulations of compressible turbulent channel flows for PP11, a heavy fluorocarbon representative of dense gases. The thermodynamic and transport property behaviour of the fluid are described by means of advanced models. A parametric study for different bulk Mach and Reynolds numbers is conducted and the results are compared with perfect-gas flows (air flows) at the same bulk conditions. The present paper is organized as follows. The governing equations and numerical set-up are provided in $\S 2$. The present DNS data and global characteristics are presented in $\S 3$. In $\S 4$, the effects of dense-gas flows on mean and fluctuating properties are explained. In $\S 5$, the profile of turbulence intensities, turbulent kinetic budgets and near-wall structures are investigated. The analysis is performed by comparing with the flows for an ideal gas. Validation test cases and details about the transport properties laws for dense gases are given in the appendices.

\section{Methodology}

\subsection{Governing equations}

In the present study we consider flows of gases in the single-phase regime, governed by the compressible Navier-Stokes equations, written in differential form as

$$
\begin{gathered}
\frac{\partial \rho}{\partial t}+\frac{\partial\left(\rho u_{j}\right)}{\partial x_{j}}=0 \\
\frac{\partial\left(\rho u_{i}\right)}{\partial t}+\frac{\partial\left(\rho u_{i} u_{j}\right)}{\partial x_{j}}+\frac{\partial p}{\partial x_{i}}-\frac{\partial \tau_{i j}}{\partial x_{j}}=\rho f_{u_{i}} \\
\frac{\partial(\rho E)}{\partial t}+\frac{\partial\left((\rho E+p) u_{j}\right)}{\partial x_{j}}-\frac{\partial\left(\tau_{i j} u_{i}\right)}{\partial x_{j}}+\frac{\partial q_{j}}{\partial x_{j}}=\rho f_{u_{i}} u_{i},
\end{gathered}
$$

where $u_{i}$ is the velocity vector, $\rho$ the density and $p$ the pressure. The specific total energy $E$ and the viscous stress tensor $\tau_{i j}$ are defined as

$$
\begin{gathered}
E \equiv e+\frac{1}{2} u_{j}^{2} \\
\tau_{i j} \equiv 2 \mu S_{i j}+\lambda \theta \delta_{i j},
\end{gathered}
$$

where $e$ is the specific internal energy, $\mu$ the dynamic viscosity, $S_{i j}=(1 / 2)\left(\left(\partial u_{i} / \partial x_{j}\right)+\right.$ $\left.\left(\partial u_{j} / \partial x_{i}\right)\right)$ is the strain rate tensor, $\theta \equiv \partial u_{k} / \partial x_{k}$ the velocity divergence or dilatation, $\delta_{i j}$ the Kronecker symbol and $\lambda=-2 \mu / 3$ the second viscosity according to Stokes' hypothesis. Finally, $q_{j}$ represents the heat flux, modelled by means of the Fourier's law:

$$
q_{j}=-\kappa \frac{\partial T}{\partial x_{j}},
$$

where $\kappa$ is the thermal conductivity. The system is supplemented by thermal and caloric equations of state, respectively:

$$
p=p(\rho, T) \quad \text { and } \quad e=e(\rho, T) .
$$


These variables satisfy the compatibility relation:

$$
e=e_{r}+\int_{T_{r}}^{T} c_{v, \infty}\left(T^{\prime}\right) \mathrm{d} T^{\prime}-\int_{\rho_{r}}^{\rho}\left[\left.T \frac{\partial p}{\partial T}\right|_{\rho}-p\right] \frac{\mathrm{d} \rho^{\prime}}{\rho^{\prime 2}},
$$

where the subscript $(\cdot)_{r}$ indicates a reference state, $c_{v, \infty}(T)$ is the specific heat at constant volume in the ideal-gas limit, and the superscript $(\cdot)^{\prime}$ denotes auxiliary integration variables. For a thermally and calorically perfect gas, equations (2.7) become:

$$
p=\rho R T \text { and } e=c_{v} T,
$$

where $c_{v}=R /(\gamma-1)=$ const., and $R=\mathcal{R} / \mathcal{M}$ with $\mathcal{R}$ the universal gas constant and $\mathcal{M}$ the gas molecular weight. Moreover, thermodynamic models relating the dynamic viscosity $\mu$ and thermal conductivity $\kappa$ to the gas temperature and pressure are needed. In the following simulations, we consider the perfluoro-perhydrophenanthrene, (chemical formula $\mathrm{C}_{14} \mathrm{~F}_{24}$ ), called hereafter PP11 (commercial name), whose thermodynamic properties are provided in table 1 (see Cramer 1989). This fluid has been often used in the literature since it exhibits a wide inversion zone and thus significant dense-gas effects. The thermodynamic behaviour of the fluid is modelled through the Martin-Hou thermal equation of state (Martin \& Hou 1955), which provides a reasonably accurate description of the gas behaviour and of the inversion zone size. Such an equation involves five virial terms and ensures high accuracy with a minimum amount of experimental information. The equation reads:

$$
p=\frac{R T}{(v-b)}+\sum_{i=2}^{5} \frac{f_{i}(T)}{(v-b)^{i}}
$$

with $b=v_{c r}\left(1-\beta / Z_{c r}\right), \beta=20.533 Z_{c r}-31.883 Z_{c r}^{2}$, and

$$
f_{i}(T)=A_{i}+B_{i} T+C_{i} \exp \left(-k T / T_{c r}\right),
$$

where $v=1 / \rho$ denotes the specific volume, $v_{c r}, T_{c r}$ and $Z_{c r}$ the critical specific volume, temperature and compressibility factor, respectively, and $k=5.475 . A_{i}, B_{i}$ and $C_{i}$ are gas-dependent coefficients that can be expressed in terms of the critical temperature and pressure, the critical compressibility factor, the Boyle temperature (which may be expressed as a function of the critical temperature) and one point on the vapour pressure curve. A power law of the form:

$$
c_{v_{\infty}}(T)=c_{v_{\infty}}\left(T_{c r}\right)\left(\frac{T}{T_{c r}}\right)^{n}
$$

is used to model variations of the low-density specific heat with temperature, where $n$ is a material-dependent parameter. The fluid viscosity and thermal conductivity are evaluated by means of the generalized laws derived by Chung et al. (1988), which contain a correction term that takes into account the strong density dependence of the transport properties in the dense-gas region. These laws, extensively used in previous works (Cramer \& Tarkenton 1992; Cramer \& Park 1999), are described in appendix A. In the modelling, the bulk viscosity $\mu_{b}=\lambda+2 \mu / 3$ is set to zero, assuming a Stokesian fluid. Since this coefficient is multiplied by the divergence of the velocity $\theta$, the hypothesis is exact in the incompressible limit. This is also 


$$
\begin{array}{ccccccccccc} 
& \mathcal{M} & T_{c r} & \rho_{c r} & p_{c r} & Z_{c r} & \omega & \xi & T_{b} & c_{v}\left(T_{c r}\right) / R & n \\
& \mathrm{~g} \mathrm{~mol}^{-1} & \mathrm{~K} & \mathrm{~kg} \mathrm{~m}^{-3} & \mathrm{MPa} & - & - & \mathrm{D} & \mathrm{K} & - & - \\
\text { PP11 }\left(\mathrm{C}_{14} \mathrm{~F}_{24}\right) & 624.11 & 650.2 & 627.14 & 1.46 & 0.2688 & 0.4963 & 0.0 & 488.15 & 97.3 & 0.5776
\end{array}
$$

TABLE 1. Thermodynamic properties of PP11: molecular weight $(\mathcal{M})$, critical temperature $\left(T_{c r}\right)$, critical density $\left(\rho_{c r}\right)$, critical pressure $\left(p_{c r}\right)$, critical compressibility factor $\left(Z_{c r}\right)$, acentric factor $(\omega)$, dipole moment of the gas phase $(\xi)$, boiling temperature $\left(T_{b}\right)$ and ratio of ideal-gas specific heat at constant volume over the gas constant $\left(c_{v}\left(T_{c r}\right) / R\right)$ at the critical point.

a very good approximation for low Mach numbers, for monoatomic gases, but can be questioned for a complex molecular fluid (Cramer \& Bahmani 2014). The study of Cramer (2012) provides some numerical estimates for the bulk viscosity of ideal gases. It shows that the latter can vary significantly with the temperature and can be as large as hundreds or thousands of times the shear viscosity, even for some common diatomic gases. Interestingly, the bulk viscosity ratio $\mu_{b} / \mu$ is a decreasing function of the temperature for most gases, except, e.g. methane $\left(\mathrm{CH}_{4}\right)$ which exhibits a peak around $T \approx 400 \mathrm{~K}\left(\mu_{b} / \mu \approx 350\right)$. Specifically, for the fluorocarbon $\mathrm{SF}_{6}$, commonly considered as a dense gas (Anderson 1991), $\mu_{b} / \mu$ decreases quickly with $T$, dropping from approximately 300 at $300 \mathrm{~K}$ to less than 50 at temperatures between 600 and $700 \mathrm{~K}$, like the ones of interest in the present study. Even if the value of $\mu_{b}$ is unknown for a dense gas such as PP11, the effect of the bulk viscosity has been tested by taking a large value $\mu_{b}=500 \mu$. The results are almost unaffected (see appendix B) so that Stokes hypothesis $\left(\mu_{b}=0\right)$ is retained in the following.

\subsection{Numerical methods}

Calculations are based on an in-house computational fluid dynamics (CFD) code (Gloerfelt \& Berland 2013) equipped with high-order dispersion relation preserving schemes. The convective flux derivatives are approximated by using a fourth-order optimized finite-difference scheme on an eleven-point stencil, described in Bogey \& Bailly (2004), while standard fourth-order finite differences are used to discretize the viscous fluxes. Non-uniform mesh sizes are taken into account by means of coordinate transforms. To ensure accuracy, mesh stretching rate is kept below 1.5\%. As a part of the algorithm, the optimized selective sixth-order filter of Bogey, De Cacqueray \& Bailly (2009) is applied in each direction in order to eliminate grid-to-grid unresolved oscillations. Finally, time integration is carried out by means of a low-storage six-step Runge-Kutta scheme optimized in the wavenumber space (Bogey \& Bailly 2004).

The numerical strategy has been widely employed for jets (Bogey \& Bailly 2009; Bogey, Marsden \& Bailly 2012) as well as turbulent boundary layers (Aubard, Gloerfelt \& Robinet 2013; Gloerfelt \& Berland 2013). The code has been preliminarily validated for turbulent channel flow configurations by comparison with reference results for both subsonic (Kim, Moin \& Moser 1987) and supersonic (Coleman et al. 1995) channel flows of a perfect gas (see appendix C).

\section{Numerical experiments of compressible channel flows}

\subsection{Set-up and definitions}

Numerical experiments are conducted for the plane channel flow configuration, i.e. the flow between two infinite parallel flat plates. This is reproduced by applying 
periodic conditions in the homogeneous streamwise $(x)$ and spanwise $(z)$ directions. Isothermal no-slip wall conditions are applied on the lower and upper walls. In order to counteract viscous friction and maintain a target bulk mass flow, the approach described in Gerolymos, Sénéchal \& Vallet (2010) and Gerolymos \& Vallet (2014) is used to compute the forcing term. Specifically, at the end of each Runge-Kutta stage, the bulk density is explicitly constrained to maintain a fixed target value, whereas a source term $f_{u_{1}}$ is injected in the streamwise momentum equation to enforce a constant mass flow. It should be noted that the density correction is equivalent to including a source term in the continuity equation. Nevertheless, this term is very small and can be neglected (Coleman et al. 1995; Huang et al. 1995; Lechner et al. 2001; Foysi et al. 2004; Morinishi et al. 2004; Wei \& Pollard 2011; Gerolymos \& Vallet 2014).

In the following, the subscripts $(\cdot)_{B},(\cdot)_{w}$ and $(\cdot)_{c}$ denote averaging over the whole computational domain, the walls and centreline, respectively; $\overline{(\cdot)}$ indicates Reynolds averaging and $(\cdot)^{\prime}$ are Reynolds fluctuations; similarly, $\widetilde{(\cdot)}$ and $(\cdot)^{\prime \prime}$ denote Favre averaging and Favre fluctuations. In the following, we also use the centreline Reynolds and Mach numbers, respectively defined as $R e_{c}=\bar{\rho}_{c} \bar{u}_{c} h / \bar{\mu}_{c}$ and $\bar{M}_{c}={\overline{u_{c}} / c_{c}}_{\text {. }}$

The DNS operating point is defined by a bulk Reynolds number $\operatorname{Re}_{B}$ and a bulk Mach number $M_{B}$, defined as:

$$
\operatorname{Re}_{B}=\frac{\rho_{B} \widetilde{u}_{B} h}{\bar{\mu}_{w}}, \quad M_{B}=\frac{\widetilde{u}_{B}}{\bar{c}_{w}},
$$

where $\rho_{B}$ is the bulk density, $\bar{\mu}_{w}$ is the dynamic viscosity and $\bar{c}_{w}$ the sound speed at the walls. In the definition of the bulk reference numbers, wall values for viscosity and speed of sound are usually considered since this greatly simplifies the imposition of the reference conditions for isothermal walls. Indeed, for a perfect-gas model, a fixed wall temperature implies $\bar{\mu}_{w}=\mu\left(T_{w}\right)$ and $\bar{c}_{w}=c\left(T_{w}\right)=\sqrt{\gamma R T_{w}}$. However, this is no longer true when using more complex thermodynamic models, since the speed of sound and the transport properties depend on both temperature and density values. In these cases, $\bar{\rho}_{w}$ is not known a priori and it is not possible to fix directly $\operatorname{Re}_{B}$ and $M_{B}$. To enforce the reference conditions, an iterative procedure has been followed: first, a preliminary calculation is run by choosing a reasonable value for the ratio $\bar{\rho}_{w} / \rho_{B}$. Once the solution is converged, the $\bar{\rho}_{w}$ obtained from the simulation is used to compute the updated values of $\bar{\mu}_{w}$ and $\bar{c}_{w}$, and the flow field is interpolated onto a new grid (adapted to the updated value of the Reynolds number). In order to achieve convergence to the desired state, about three to four iterative cycles are needed.

We recall the definition of the standard wall coordinates and friction Reynolds number

$$
y^{+}=\frac{\bar{\rho}_{w} u_{\tau}\left(y-y_{w}\right)}{\bar{\mu}_{w}} ; \quad \operatorname{Re}_{\tau_{w}}=\frac{\bar{\rho}_{w} u_{\tau} h}{\bar{\mu}_{w}},
$$

where $u_{\tau}=\sqrt{\tau_{w} / \bar{\rho}_{w}}$ is the friction velocity, $\tau_{w}={\overline{(\mu(\partial u / \partial y))_{w}}}_{w}$ being the shear stress at the wall. It is widely known that the Reynolds number based on the friction velocity does not correctly represent the effect of rapid wall-normal variations for density and viscosity profiles in presence of high compressibility effects. Huang et al. (1995) proposed an empirical semi-local scaling based on both wall and local mean quantities:

$$
y^{*}=\frac{\bar{\rho}(y) u_{\tau}^{*}\left(y-y_{w}\right)}{\bar{\mu}(y)} ; \quad \operatorname{Re}_{\tau}^{*}(y)=\operatorname{Re}_{\tau_{w}} \sqrt{\frac{\bar{\rho}(y)}{\bar{\rho}_{w}}} \frac{\bar{\mu}_{w}}{\bar{\mu}(y)},
$$


with $u_{\tau}^{*}=\sqrt{\tau_{w} / \bar{\rho}(y)}$ the semi-local friction velocity. This mixed scaling has proven to give quite satisfactory results in collapsing first- and second-order moments (Foysi et al. 2004; Morinishi et al. 2004; Modesti \& Pirozzoli 2016) obtained from a wide range of $M_{B}$ and $R e_{B}$. Recently, Patel et al. (2015) and Trettel \& Larsson (2016) have developed a mathematical framework giving a theoretical basis to the semi-local scaling. Even if this scaling was initially introduced in order to take into account the effects of compressibility, Patel et al. (2015) assessed its validity also for variableproperty flows, since it inherently considers the evolution of transport properties and their relation with thermodynamic quantities.

\subsection{Description of the computed cases}

Dense-gas effects are evaluated by means of a parametric study at three bulk Reynolds numbers (namely, $R e_{B}=3000,7000$ and 12000) and three bulk Mach numbers $\left(M_{B}=1.5,2.25\right.$ and 3.0). For PP11 cases, the bulk density was imposed to be $\rho_{B}=0.618 \rho_{c r}$ and the wall temperature was fixed to $T_{w}=1.01 T_{c r}$. The density value ensures that the flow evolves in the dense-gas region, whereas the slightly supercritical wall temperature condition avoids the occurrence of a two-phase flow. It should be noted that for these conditions it is not possible to obtain negative values of the derivative of gas dynamics $\Gamma$, so we should not expect BZT phenomena.

In the following, we refer to each simulation with a unique tag of the form $\mathrm{XM} \alpha \mathrm{R} \beta$, where the first letter indicates the fluid (A for air and P for PP11), $\alpha$ refers to $M_{B}\left(\alpha=1,2,3\right.$ for $M_{B}=1.5,2.25,3$, respectively) and $\beta$ to $\operatorname{Re}_{B}(\beta=3,7,12$ for $R e_{B}=3000,7000,12000$, respectively). For all the cases, the computational domain has dimensions $L_{x} \times L_{y} \times L_{z}=8 \pi h \times 2 h \times 2 \pi h$, with $h$ the channel half-height. Note that the dimension in the streamwise direction is greater than the one used in the previous compressible channel DNS (Coleman et al. 1995; Lechner et al. 2001; Morinishi et al. 2004; Wei \& Pollard 2011) in order to ensure uncorrelated inlet and outlet quantities for high- $M_{B}$ cases. The computational grids are chosen in order to provide a good spatial resolution in all directions. Specifically, the chosen spatial resolutions, expressed in semi-local units, are in the ranges $\Delta x^{*} \in[10,16]$, $\Delta y_{w}^{*} \in[0.5,0.8], \Delta z^{*} \in[4,6]$, according to the considered flow conditions. The spatial resolution is also evaluated with respect to the wall-normal distribution of the Kolmogorov length scale $\eta=\left((\bar{\mu} / \bar{\rho})^{3} \bar{\rho} / \bar{\varepsilon}\right)^{0.25}$, where $\bar{\varepsilon}$ is the turbulent kinetic energy dissipation. According to Zonta et al. (2012) and Lee et al. (2013), the resolution requirements are $\Delta x<12 \eta, \Delta y<2 \eta$ and $\Delta z<6 \eta$.

The resolutions obtained for the present cases are reported in table 3 . Since a complex equation of state is used for real gases and higher values of the effective Reynolds number are reached, a grid sensitivity study has been conducted and is reported in appendix D. Simulations with PP11 require higher resolution than air to achieve grid convergence of second-order statistics, and the chosen grids in table 3 have quite satisfactory resolution. In our simulations, the overall number of grid points varies between $3 \times 10^{6}$ and $1.2 \times 10^{9}$.

Table 3 summarizes the conditions used for the parametric study. Wall friction Reynolds numbers range from 218.7 to 1017 for air, and from 191.3 to 692.8 for PP11. The semi-local scaling drastically changes the predictions in terms of friction Reynolds number. For air, the friction Reynolds number at the centreline $R e_{\tau, c}^{*}=R e_{\tau}^{*}(h)$ goes from 88.7 to 324.7 , the drop for each case being roughly proportional to $M_{B}$. For PP11, an opposite behaviour is observed: $R e_{\tau, c}^{*}$ is higher than $R e_{\tau_{w}}$ in each case, and the deviations increase with $M_{B}$. This is due to the different 


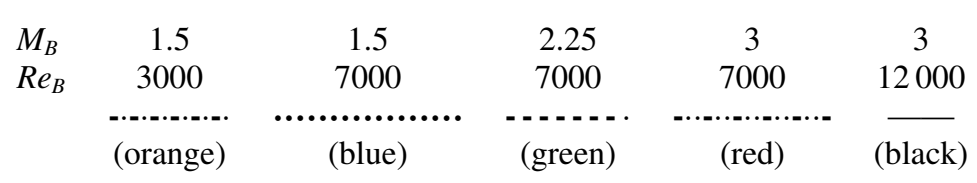

TABLE 2. Line styles for the employed DNS cases.

behaviour of the transport properties as it will be explained later. Thus, contrarily to air, the resolution requirements for PP11 are more severe in semi-local scaling than in the classical wall scaling. However, as reported in table 3 and appendix D, the chosen grids ensure a good resolution in each case. Once $R e_{\tau}^{*}$ is converged, statistics are collected every ten computational time steps, with $\Delta t^{+} \approx 0.02-0.03$ in wall units for all the simulations and during an observation time $t_{o b s}^{+}$in the range 1000-3000.

\section{Influence of dense-gas effects on flow properties}

In this section we first present results for general flow properties and their dependency on the bulk parameters and fluid type. Then, we analyse cross-wise profiles of the first-order statistics. For this purpose, we initially assess different wall scalings, and then focus more specifically on the variations of average thermodynamic properties across the channel, to highlight the peculiar behaviour of dense-gas flows compared with air flows. For the sake of clarity, we investigate the role of compressibility effects by focusing on cases characterized by $R e_{B}=7000$ and various Mach numbers. The Reynolds number influence is also studied by considering sets of simulations M1R3 and M3R12. We complete the analysis by discussing dense-gas effects on second-order statistics of the thermodynamic and transport properties. Second-order moments of the velocity fields and, more generally, effects on turbulence structure are discussed in $\S 5$. For these sets of results, each case is uniquely identified with the line style defined in table 2 , unless otherwise stated.

\subsection{Global flow properties}

Table 4 summarizes the main characteristic values of the simulations. Figure 1 provides a first insight into the behaviour of PP11 cases compared to air cases. In this figure, $M_{B}$ and $\bar{T}_{c}^{+}=\bar{T}_{c} / T_{w}$ are plotted as functions of the average centreline Mach number $\bar{M}_{c}$. Values obtained for air cases are in good agreement with reference DNS (Coleman et al. 1995; Foysi et al. 2004; Gerolymos \& Vallet 2014). For $M_{B} \geqslant 1.5$, $\bar{M}_{c}$ is systematically lower than $M_{B}$, since the walls are cooler than the channel core. Indeed for perfect gases, $\bar{M}_{c}$ increases nonlinearly since $\bar{T}_{c}$ grows rapidly and $\bar{c} \propto \sqrt{\bar{T}}$. The average centreline temperature in figure $1(b)$ is approximately 1.4 times higher than the wall temperature for AM1R3 and more than 2.5 times higher for AM3R3 due to the significant increase of the viscous heating $\overline{\tau_{i j} S_{i j}}$. The dependence on the Reynolds number is weak on the considered range, with slightly lower values of $\bar{T}_{c}^{+}$for higher $R e_{B}$, which is more clearly appreciated for the highest Mach number conditions. PP11 flows exhibit a rather different behaviour since the molecular complexity of dense gases implies high values of the specific heat $c_{v} / R$ and, therefore, the coupling between the thermal and dynamic fields becomes smaller and smaller. Inspection of figure $1(b)$ indeed shows that the centreline temperature is almost equal to the wall value for any choice of the Mach and Reynolds numbers. 


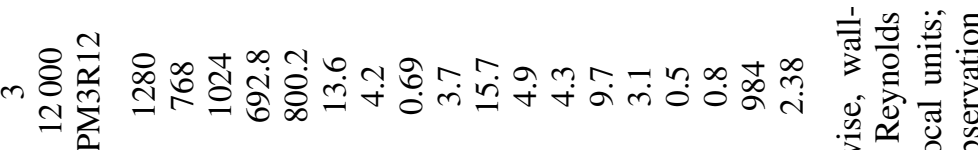

m

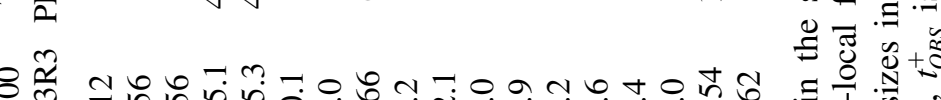

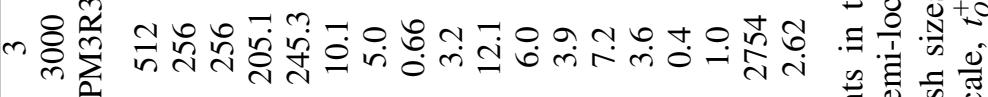

$=$ そ) 元

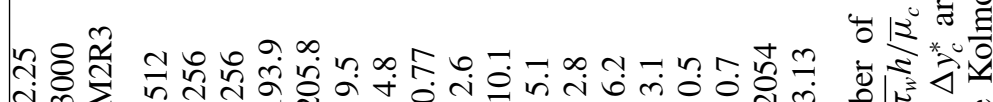

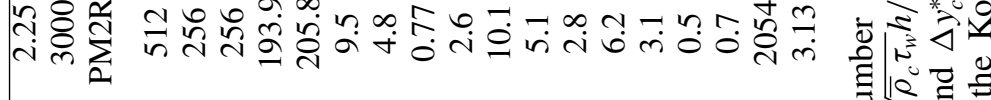

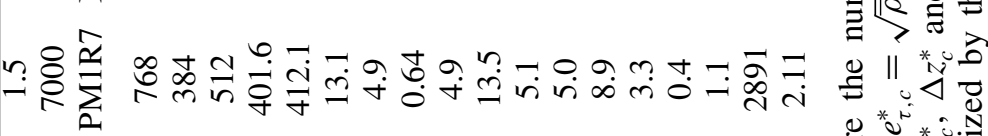

先 v ৩

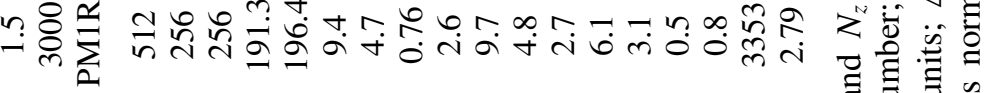

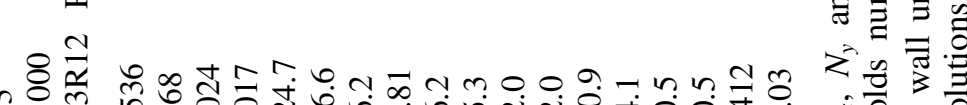

일 m

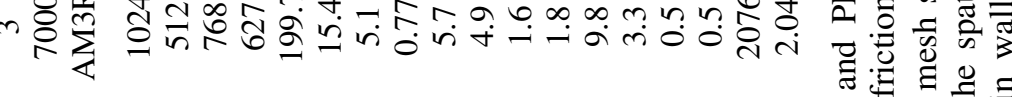

m

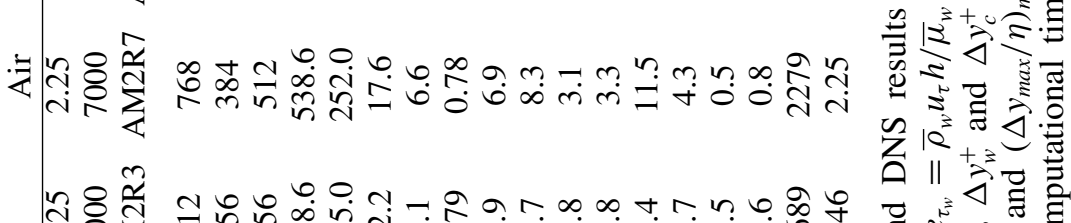

응

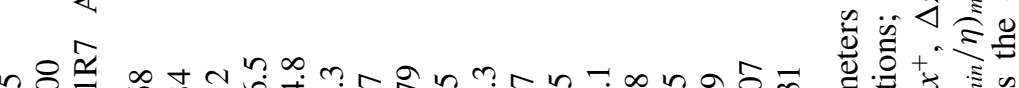

传运

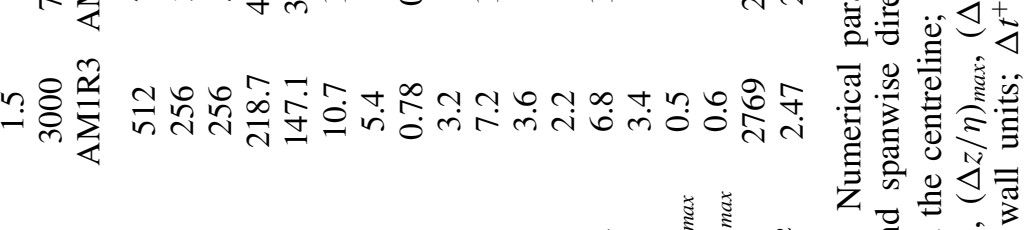

尊导

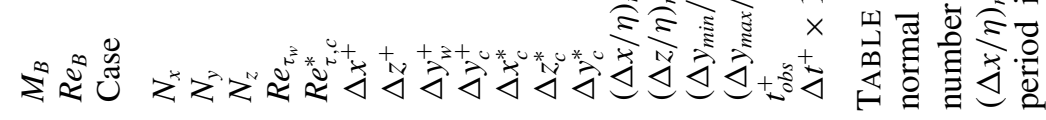




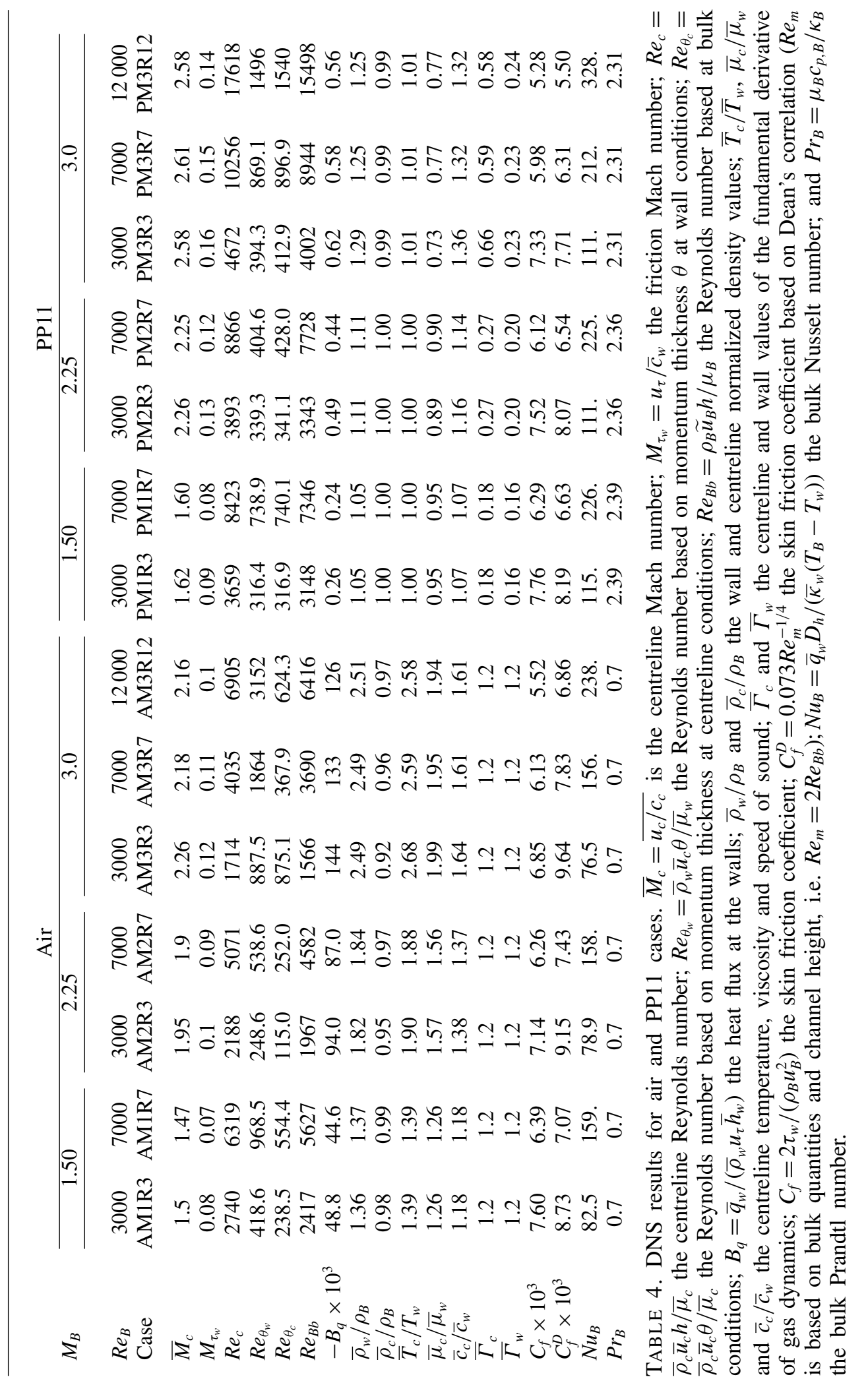



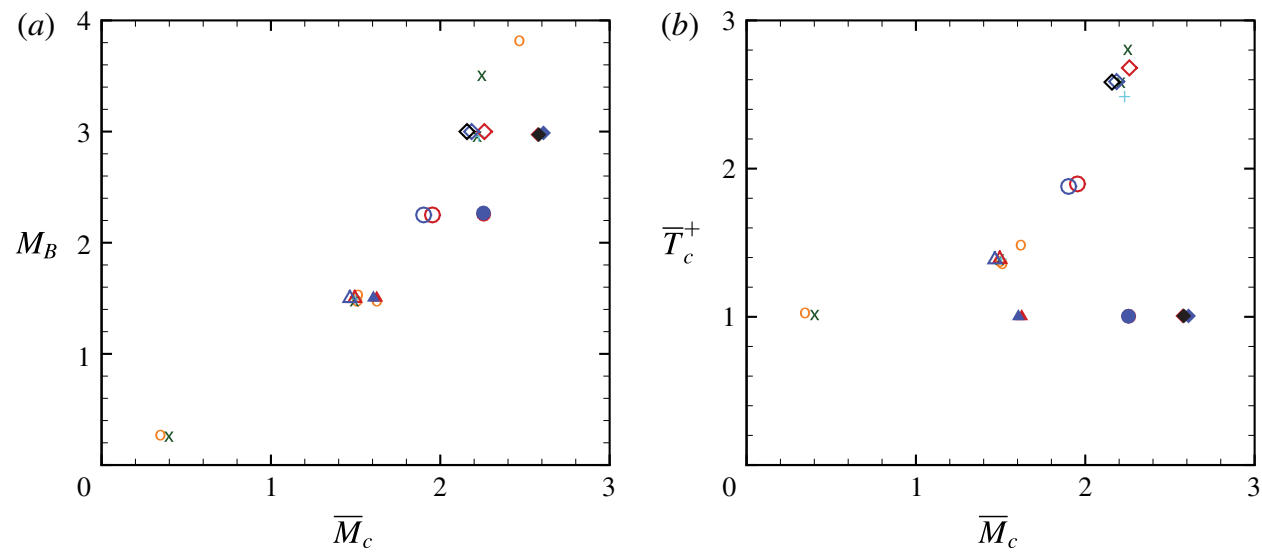

Figure 1. (Colour online) Evolution of $M_{B}(a)$ and $\bar{T}_{c}^{+}(b)$ as a function of $\bar{M}_{c}$ for different cases. Open symbols: air; filled symbols: PP11. Symbol shapes: $\mathbf{\Delta}: M_{B}=1.5$, ๑: $M_{B}=2.25, \diamond: M_{B}=3$. Symbol colours (online version): red, $R e_{B}=3000$; blue, $R e_{B}=7000$; black, $R e_{B}=12000$. + (cyan) Coleman et al. (1995), × (green) Foysi et al. (2004), $\bigcirc$ (orange) Gerolymos \& Vallet (2014).

Nevertheless, the evolution of $\bar{M}_{c}$ changes significantly because in dense gases the speed of sound depends also on density, which decreases moving from the wall to the centreline. In turn, $\bar{c}_{c}$ increases leading to lower values of $\bar{M}_{c}$.

For practical applications, it is interesting to investigate the modifications of momentum and heat transfer coefficients when working with a dense gas. For that purpose, we consider the evolutions of the skin friction coefficient $C_{f}$ and the bulk Nusselt number $N u_{B}$, respectively defined as

$$
C_{f}=\frac{\tau_{w}}{\frac{1}{2} \rho_{B} u_{B}^{2}} ; \quad N u_{B}=\frac{\bar{q}_{w} D_{h}}{\bar{\kappa}_{w}\left(T_{B}-T_{w}\right)},
$$

which are depicted in figure 2. $D_{h}$ denotes the hydraulic diameter, $D_{h}=4 h$ for a flat channel. The values predicted by the correlation of Dean (1978), i.e. $C_{f}^{D}=0.073 R e_{m}^{-1 / 4}$, are superimposed in figure 2(a). The correlation has been established in the incompressible regime for $R e_{m}>6000$, where $R e_{m}$ is the Reynolds number based on bulk quantities and channel height, i.e. $R e_{m}=2 R e_{B b}=\rho_{B} \widetilde{u}_{B} 2 h / \mu_{B}$. For the dense-gas cases, the computed skin friction coefficients are slightly lower than the values predicted by Dean's correlation, but follow closely the trend with respect to the bulk Reynolds number. The deviations are greater for air ideal flows with an underestimation more pronounced for higher Mach numbers. Some authors (Moneghan 1953; Huang, Bradshaw \& Coakley 1993) have proposed compressibility corrections, which work well for turbulent boundary layers over adiabatic walls. A similar extension for channel flows is not without ambiguities since the definition of the freestream values and of the dynamic pressure for normalization are more arbitrary. It can however be concluded that in dense-gas flow decoupling of dynamic and thermal effects dominates, so that Mach number effects are less influential on the skin friction coefficient than in air flows, and Dean's formula yields a reasonable correlation.

In a similar manner, the Nusselt number is compared in figure $2(b)$ with a classical empirical correlation, commonly used to estimate heat transfer in hydraulic pipes 

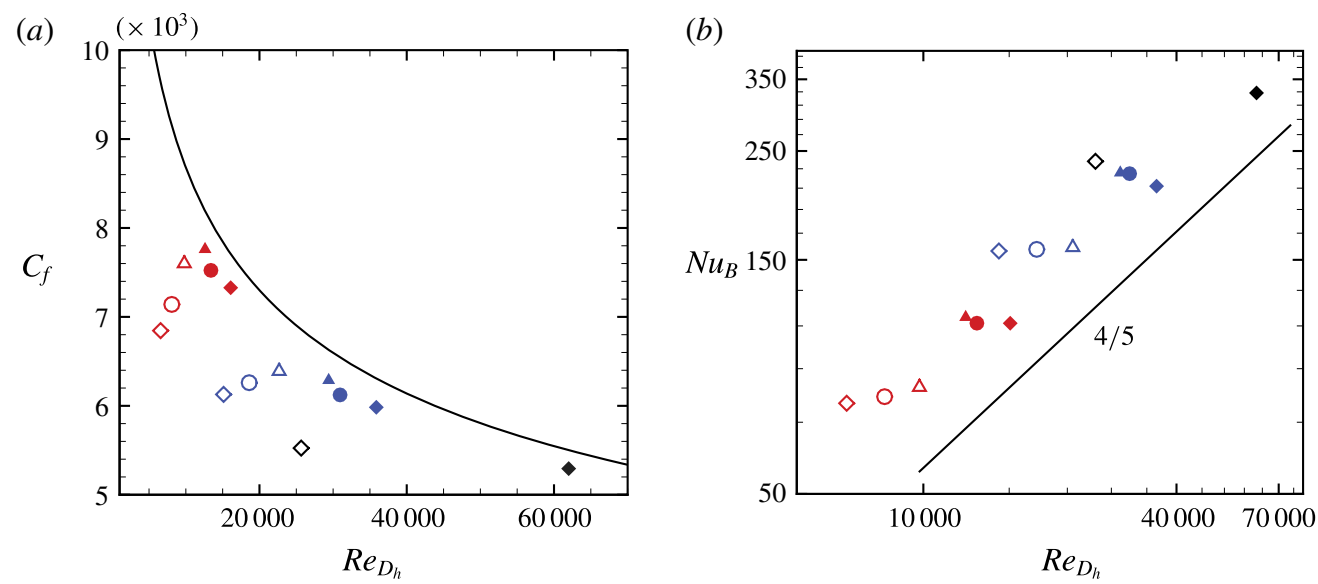

FIGURE 2. (Colour online) Evolutions of $C_{f}(a)$ and $N u_{B}(b)$ as a function of $R e_{D_{h}}$ for different cases. Same legend as figure 1.

(Incropera \& DeWitt 2007; Zonta 2013). Specifically, we use the Sieder-Tate correlation, $N u_{B}^{S T}=0.027 \operatorname{Re}_{D_{h}}^{4 / 5} \operatorname{Pr}_{B}^{1 / 3}\left(\mu_{B} / \mu_{w}\right)^{0.14}$ (Sieder \& Tate 1936). Here, the Reynolds number at bulk conditions is based on the hydraulic diameter $D_{h}$, i.e. $R e_{D_{h}}=2 R e_{m}=4 R e_{B b}$. This formula is an extension of the Dittus-Boelter/Colburn correlations including the viscosity dependence due to temperature change between the bulk average temperature and wall temperature. Figure $2(b)$ shows that the power-law exponent of 0.8 captures well the Reynolds number dependence for both fluids. The higher values obtained for dense-gas cases are explained by the Prandtl number dependence, which is constant equal to 0.7 for air and varies between 2.3 and 2.4 for PP11. The enhanced heat capacities of dense gases reduce the heat conduction. A Mach number effect can also be noticed, leading to lower Nusselt numbers when the Mach number increases. This compressibility effect, more visible for air flows, is not taken into account by the empirical correlation. The predicted values of $C_{f}$ and $N u_{B}$ are reported in table 4 . The heat flux towards the walls $B_{q}=\bar{q}_{w} /\left(\bar{\rho}_{w} u_{\tau} \bar{h}_{w}\right)$, with $\bar{h}_{w}$ the specific enthalpy at the wall, is also given. Its order of magnitude is $O\left(10^{0}-10^{1}\right)$ for air and $O\left(10^{-4}\right)$ for PP11 flows. Hence, for dense gases, isothermal walls translate also into quasi-adiabatic conditions. Also given in table 4, the wall and centreline mean values of the fundamental derivative $\bar{\Gamma}_{w}$ and $\bar{\Gamma}_{c}$ are globally lower than unity. Their increase with $M_{B}$ is mainly associated with a higher dispersion of the instantaneous thermodynamic states.

\subsection{Scalings and first-order statistics}

Figure 3 displays the mean streamwise velocity profiles for air $(a-c)$ and PP11 flows $(d-f)$. It is known that the classical incompressible scaling $(a, d)$ fails in collapsing the velocity profiles when compressibility effects are present. In particular, predictions get worse in presence of a substantial heat flux towards the walls. Several attempts have been made in the past in order to derive a transformation able to collapse the compressible velocity profiles into an universal law of the wall for a wide range of Mach numbers. For non-hypersonic flows, Van Driest (1951) developed the 
(a)

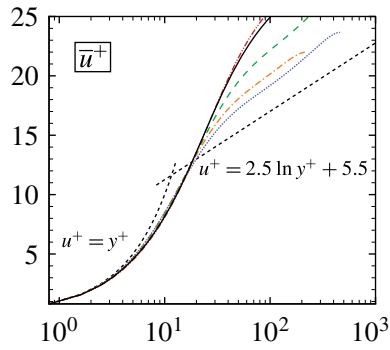

(d)

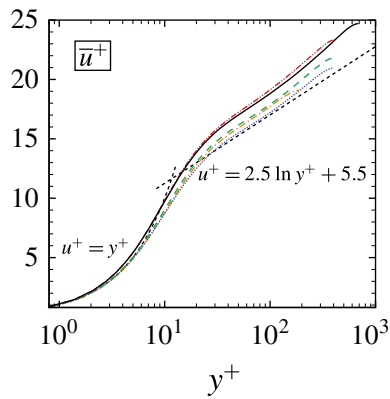

(b) 25

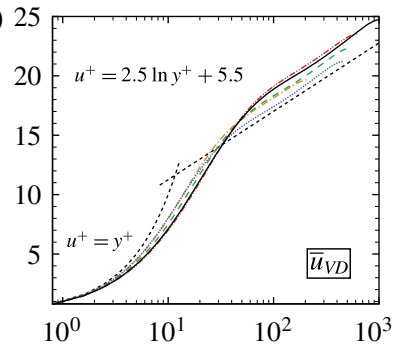

(c)

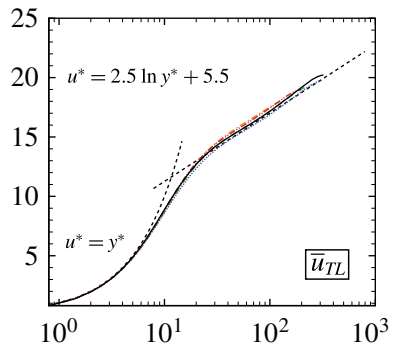

(e)

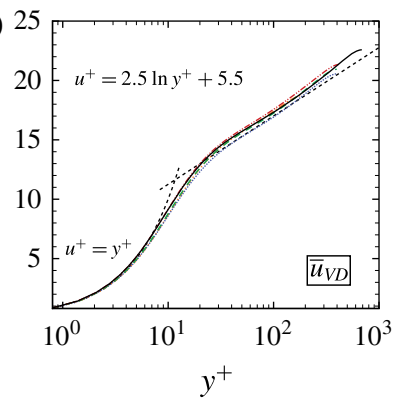

$(f) 25$

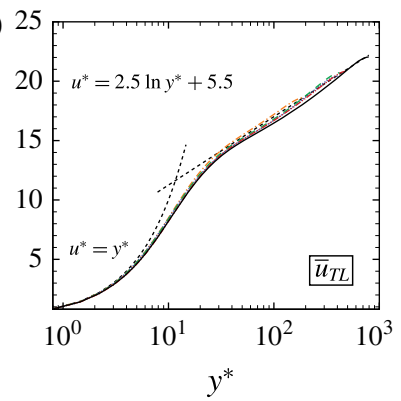

FIgURE 3. (Colour online) Scalings for the law of the wall for air (top) and PP11 (bottom). (a,d) Classical incompressible scaling $\bar{u}^{+} ;(b, e)$ Van Driest transformation $\bar{u}_{V D}$; $(c, f)$ Trettel-Larsson transformation $\bar{u}_{T L}$ in semi-local coordinates. For the meaning of the line styles in this and subsequent figures, see table 2.

transformation:

$$
\bar{u}_{V D}=\int_{0}^{u^{+}} \sqrt{\frac{\bar{\rho}}{\bar{\rho}_{w}}} \mathrm{~d} u^{+},
$$

which takes into account mean-density variations and translates the compressible profile into an equivalent 'constant-density' profile keeping the same wall-normal coordinate $y^{+}$. The Van Driest transformation provides a substantial improvement of the scaling $(b, e)$, even if it departs from the log law as $M_{B}$ increases. The size of the buffer layer increases rapidly for air since the beginning of the logarithmic zone moves towards higher $y^{+}$values (from $y^{+} \approx 20$ for AM1R7 to $y^{+} \approx 70$ for AM3R12). On the other hand, for dense gases, the heat flux is greatly reduced and $\bar{u}_{V D}$-scaling provides quite satisfactory results. Indeed, this scaling was found to behave well for adiabatic walls (Guarini et al. 2000; Pirozzoli, Grasso \& Gatski 2004; Pirozzoli \& Bernardini 2011). Modifications for the value of the intercept of the log-law velocity profile, $u^{+}=1 / \kappa_{V K} \log y^{+}+C$ were proposed by Bradshaw (1977), yielding empirical laws that relate $C$ to the friction Mach number $M_{\tau}$ and to the heat flux $B_{q}$. Modesti \& Pirozzoli (2016) have recently provided a thorough review of the main scalings available in the literature, and have shown that the transformation proposed by Trettel \& Larsson (2016),

$$
\bar{u}_{T L}=\int_{0}^{u^{+}} \sqrt{\frac{\bar{\rho}}{\bar{\rho}_{w}}}\left[1+\frac{1}{2} \frac{1}{\bar{\rho}} \frac{\mathrm{d} \bar{\rho}}{\mathrm{d} y} y-\frac{1}{\bar{\mu}} \frac{\mathrm{d} \bar{\mu}}{\mathrm{d} y} y\right] \mathrm{d} u^{+}
$$

accurately reproduces the mean velocity profile even for cases with high wall heat flux. Panels $(c, f)$ confirm its validity even for dense gases. Note that, with this 
(a) 1.0

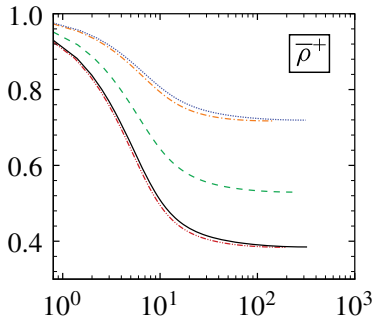

(d)

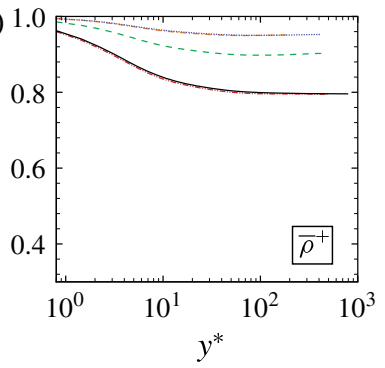

(b)

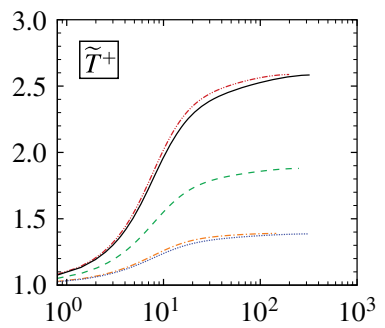

(e)

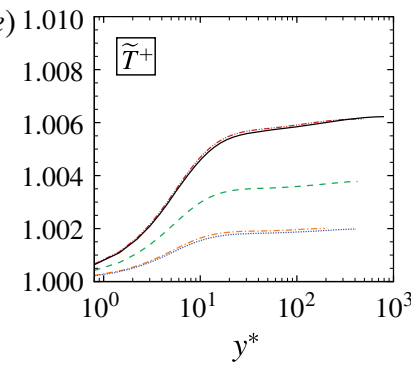

(c)

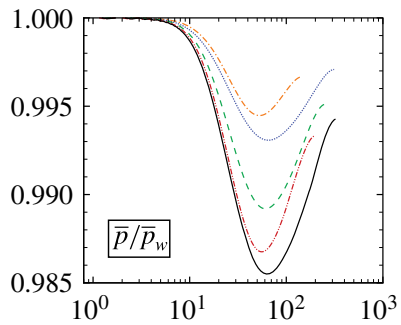

(f)

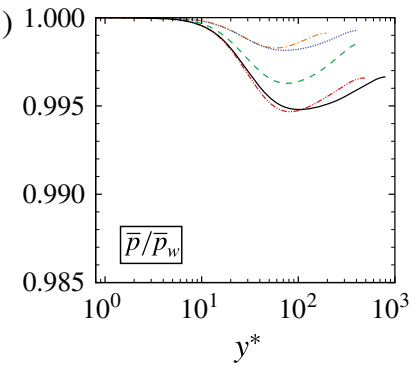

FIgURE 4. (Colour online) Profiles of density $\bar{\rho}^{+}$, temperature $\widetilde{T}^{+}$and pressure $\bar{p} / \bar{p}_{w}$ for air (top) and PP11 (bottom) cases. Same legend as in table 2.

transformation, the wall-normal distance is normalized according to the semi-local scaling $y^{*}$.

Figure 4 shows the density, temperature and pressure profiles normalized with wall quantities. The semi-local scaling $y^{*}$ is retained from hereafter as the standard scaling for comparing perfect- and dense-gas cases. The rise of wall density values for increasing $M_{B}$ is significant for air and less pronounced for PP11 flows. For instance, $\bar{\rho} / \rho_{B}=2.51$ for AM3R12 and 1.25 for PM3R12 (see values in table 4). Mean density $\bar{\rho}^{+}$decreases towards the centreline, with minimum values $\min _{y}\left\{\bar{\rho}_{c}{ }^{+}\right\} \approx 0.39$ for AM3R3 and $\approx 0.77$ for PM3R3. An eventual influence of the Reynolds number is hidden by the strong dependence on $\bar{M}_{c}$, noticeable for both $\bar{\rho}^{+}$and $\widetilde{T}^{+}$. As prefigured by figure $1(b)$, temperature profiles are quite different, with increases lower than $1 \%$ of the wall temperature for PP11 flows. Pressure is roughly constant across the channel. More precisely, it exhibits a constant value in the viscous sublayer, then it slightly decreases in the buffer layer reaching a minimum in the logarithmic zone, and afterwards increases in the outer region. For air, the relationship $\bar{p} / \bar{p}_{w}=\bar{\rho}^{+} \widetilde{T}^{+} \lesssim 1$ (Gerolymos \& Vallet 2014) holds in each case. The minimum values for air flows are up to three times higher than for PP11 flows, with $\max _{y}\left\{\left(\bar{p}-\bar{p}_{w}\right) / \bar{p}_{w}\right\} \approx 1.5 \%$ for AM3R12 and $0.5 \%$ for PM3R12. Although pressure is nearly constant, its mean value can be directly related to the turbulent fluctuations. In fact, by applying the Favre averaging to the Navier-Stokes equations, one obtains for the mean equation in the $y$-direction (Huang et al. 1995):

$$
\frac{\partial \bar{p}}{\partial y}=\frac{\partial \bar{\tau}_{y y}}{\partial y}-\frac{\partial \bar{\rho} \widetilde{v^{\prime \prime} v^{\prime \prime}}}{\partial y} .
$$

For compressible flows, the second term is not equal to zero but remains much lower than the other two. As a consequence, the minimum value of $\bar{p}$ coincides with the maximum of the wall-normal Reynolds stresses, as it will be shown later. 
(a)
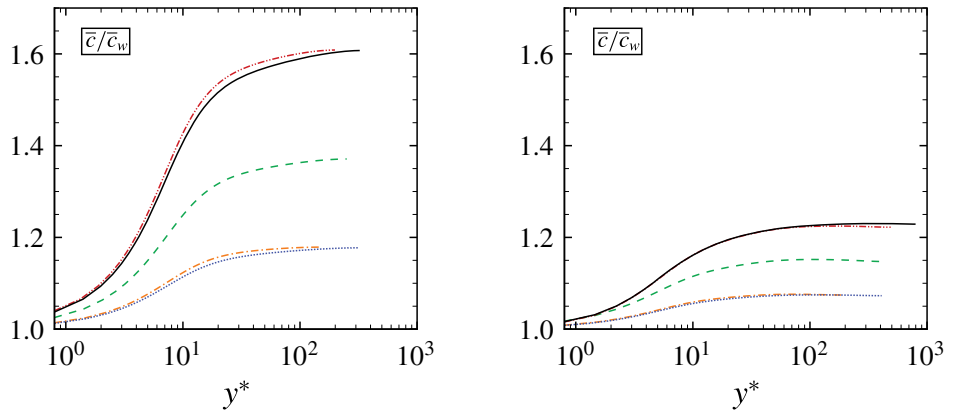

FIgURE 5. (Colour online) Mean profiles of the speed of sound $\bar{c} / \bar{c}_{w}$ for air $(a)$ and PP11 (b) flows.

(a)

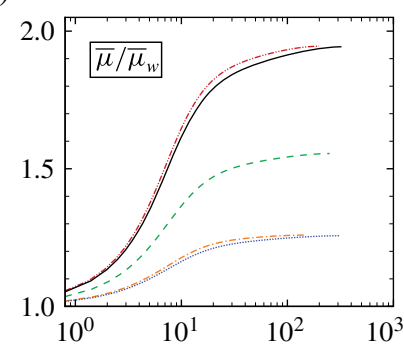

(d)

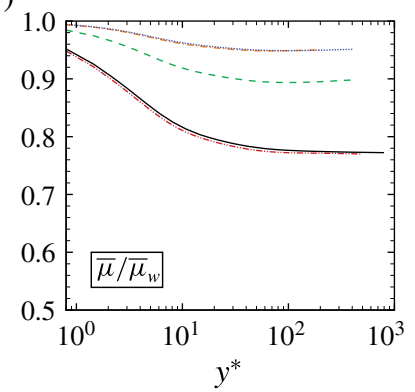

(b)

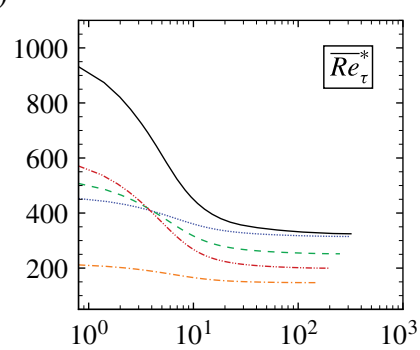

(e)

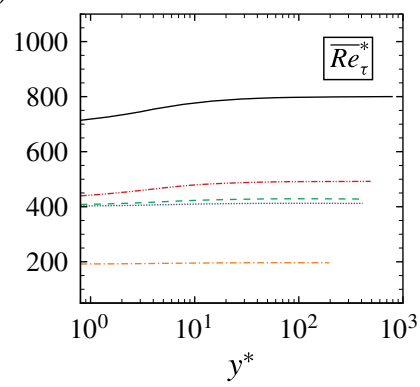

(c) $\quad\left(\times 10^{3}\right)$

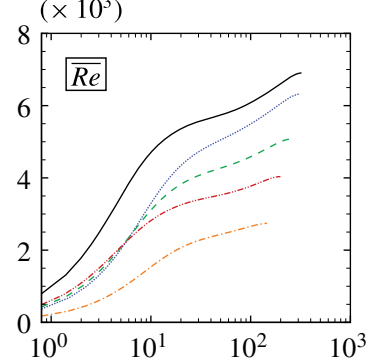

(f) $\left(\times 10^{3}\right)$

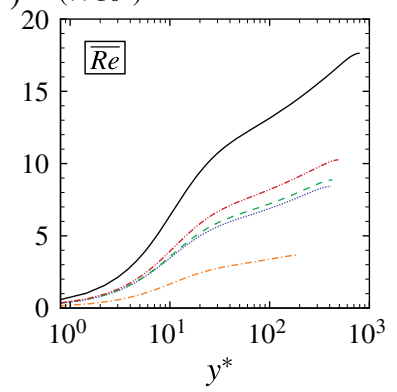

FIgURE 6. (Colour online) Mean profiles of the viscosity $\bar{\mu} / \bar{\mu}_{w}$, the friction Reynolds number $R e_{\tau}^{*}$ and the Reynolds number $\overline{R e}$ for air $(a-c)$ and PP11 $(d-f)$ flows.

Figure 5 shows the mean profiles of the speed of sound across the channel. Sound speed variations across the channel are related to temperature changes for air $\left(\bar{c} / \bar{c}_{w}=\sqrt{\bar{T}^{+}}\right)$, whereas they follow density variations for PP11. We notice that relative variations of the speed of sound are much smaller for PP11 (approximately $20 \%$ against $60 \%$ for air), leading to higher values of $\bar{M}$ compared to air flows.

The most striking differences between perfect- and dense-gas flow cases are highlighted in figure 6, presenting the profiles of viscosity, semi-local friction Reynolds number $R e_{\tau}^{*}$ and average Reynolds number $\overline{R e}$. For air flows, $\bar{\mu} / \bar{\mu}_{w}$ increases towards the centreline (gas-like behaviour), with a maximum deviation in AM3R3 for which $\max _{y}\left\{\bar{\mu} / \bar{\mu}_{w}\right\} \approx 2$. For PP11 flows, the ratio decreases (following approximately the $\bar{\rho}^{+}$profile) up to $\min _{y}\left\{\bar{\mu} / \bar{\mu}_{w}\right\} \approx 0.75$ for PM3R3, which is a liquid-like behaviour. 

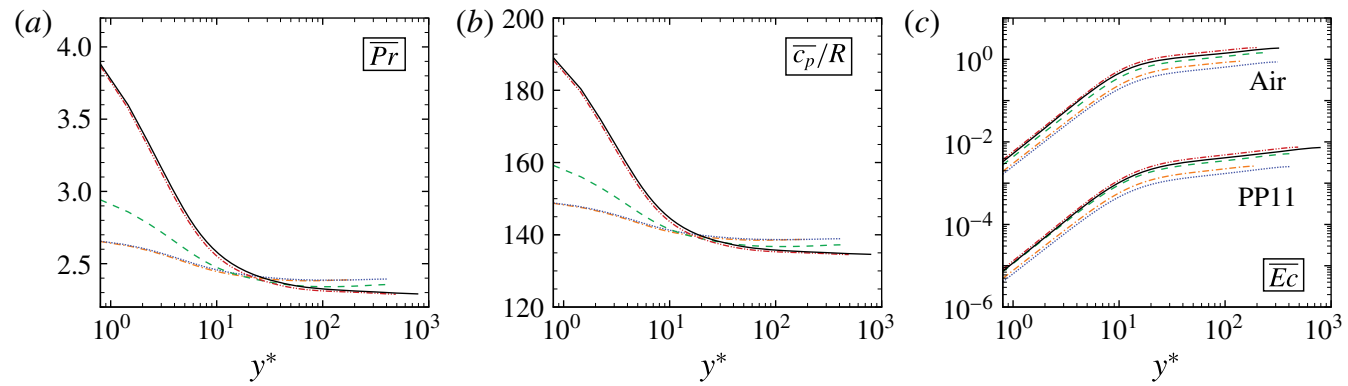

Figure 7. (Colour online) Mean profiles of the Prandtl number $\overline{\operatorname{Pr}}(a)$, specific heat at constant pressure $\overline{c_{p}} / R(b)$ for PP11 and Eckert number $\overline{E c}(c)$ for air and PP11.

Viscosity largely influences the profiles of $\overline{R e}$ and in particular the centreline values, that tend to be considerably larger for dense gases. The evolution of $R e_{\tau}^{*}$ follows opposite behaviours for the two fluids, due to the aforementioned evolutions of transport properties. For air flows, it decreases rapidly up to the logarithmic layer and then more slowly in the outer region. For a given $R e_{B}$, a higher $M_{B}$ leads to higher values of $R e_{\tau}^{*}$ near the wall, since $\bar{\mu}_{w}$ is the same whereas $\bar{\rho}_{w}$ and $\overline{(\partial u / \partial y)_{w}}$ are increasing functions of the Mach number. At greater distance from the wall, $R e_{\tau}^{*}$ decreases even more quickly due to the strong temperature gradients in the wall-normal direction. Outside of the viscous sublayer, $R e_{\tau}^{*}$ is considerably lower than the near-wall one for the higher $M_{B}$ cases, due to the combined effect of a rapid decrease of local density and an increase of local viscosity. Specifically, the centreline value may drop below the one obtained for lower Mach number cases, for which the fluid properties vary less. For PP11 flows, $R e_{\tau}^{*}$ exhibits an opposite behaviour, namely, it increases when moving from the wall to the centreline. The overall variations, of the order of $12 \%$ at most for high Mach number cases, are anyway much smaller than in the perfect gas (variations up to $60 \%$ ). When increasing the wall distance, $R e_{\tau}^{*}$ slightly increases up to the buffer layer and remains roughly constant afterwards. Due to the increasing reduction of centreline viscosity for higher Mach number cases, the growth of $R e_{\tau}^{*}$ is enhanced when $M_{B}$ becomes higher. The liquid-like viscosity behaviour clearly affects the profile of the local Reynolds number $\overline{R e}$ (panel $f$ ) compared to the perfect gas (panel $c$ ). For instance, at the centreline, $\overline{R e}_{c}$ is about 2.5 times higher for PM3R12 than for AM3R12 results.

A direct consequence of the constant Prandtl number assumption in the ideal model is that $\bar{\kappa} / \bar{\kappa}_{w}=\bar{\mu} / \bar{\mu}_{w}$ for air. In PP11 simulations, variations of the thermal conductivity across the channel are smaller than the ones of viscosity $\left(\min _{y}\left\{\bar{\kappa} / \bar{\kappa}_{w}\right\} \approx 0.95\right)$, nevertheless the ratio of the two transport properties remains roughly constant and $\bar{\mu} / \bar{\kappa} \approx 1.25$ throughout the channel. Thus, the Prandtl number profile (figure $7 a$ ) is essentially driven by the normalized specific heat at constant pressure $\overline{c_{p}} / R$ (figure $7 b$ ) which, in turn, follows a trend similar to the density profile (i.e. decreases with the wall distance). As pointed out in the above, the latter exhibit a strong dependency on $M_{B}$ in the near-wall region, which is then fed back to $\overline{P r}$. Specifically, the wall Prandtl number increases by approximately 50\% when doubling the bulk Mach number and is almost insensitive to the Reynolds number. In the outer region, $\overline{\operatorname{Pr}} \approx 2.3-2.4$ for all dense-gas cases. On the other hand, the high values of $\overline{c_{p}} / R$ for dense gases lead to very small values of the average Eckert number, $\overline{E c}=\overline{u^{2} / c_{p} T}$, a parameter that is representative of the degree of coupling between thermal and kinematic effects. We 
(a)

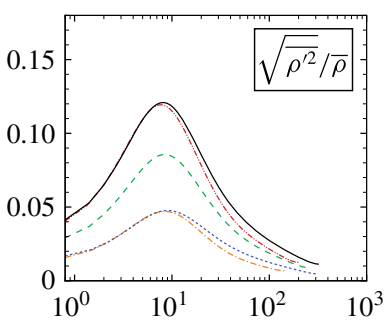

(d)

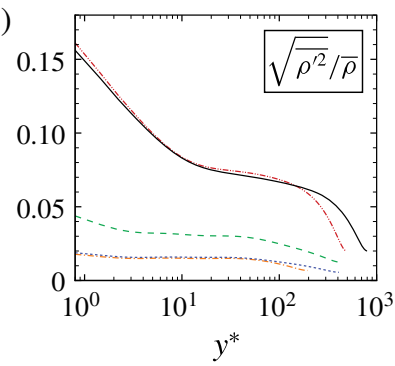

(b)

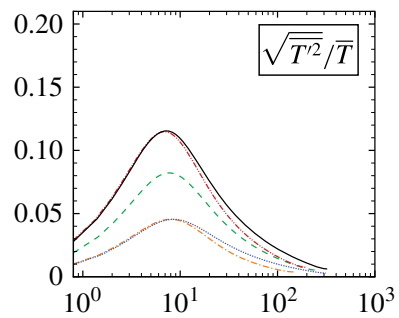

(e) 0.00

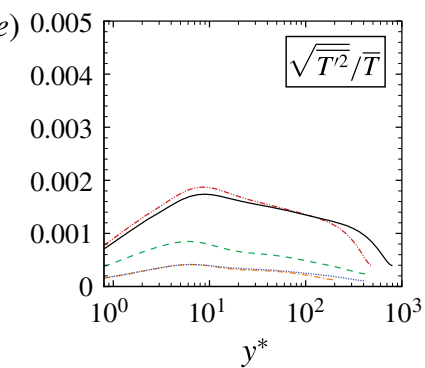

(c)

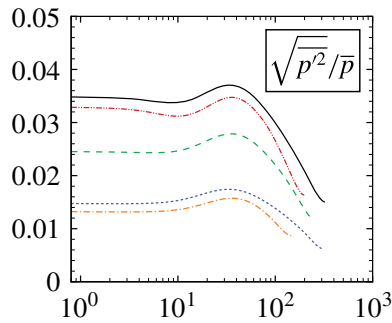

Figure 8. (Colour online) Profiles of the normalized root mean square density $\rho_{\text {rms }} / \bar{\rho}=$ $\sqrt{\overline{\rho^{\prime 2}}} / \bar{\rho}(a, d)$, temperature $T_{r m s} / \bar{T}=\sqrt{\overline{T^{\prime 2}}} / \bar{T}(b, e)$ and pressure $p_{r m s} / \bar{p}=\sqrt{\overline{p^{\prime 2}}} / \bar{p}(c, f)$ for air flows $(a-c)$ and PP11 flows $(d-f)$.

recall that for air flows, $\overline{c_{p}} / R=c_{p} / R=3.5$, which is two orders of magnitude lower than the mean values obtained with PP11. In figure $7(c), \overline{E c}$ exhibits a similar trend for both fluids, with two logarithmic zones connected near $y^{*} \approx 15$, but the curves are shifted by approximately two orders of magnitude. In fact, the centreline values are of the order of $O(1)$ for air and $O\left(10^{-2}\right)$ for PP11 cases.

\subsection{Second-order statistics of the thermodynamic and transport properties}

Root mean square (r.m.s.) values for the density, temperature and pressure are shown in figure 8. Gerolymos \& Vallet (2014) have shown that for air flows $O\left(T_{r m s} / \bar{T}\right)=$ $O\left(\rho_{r m s} / \bar{\rho}\right)=O\left(p_{r m s} / \bar{p}\right)$, regardless of the $y^{*}$ location, Mach and Reynolds numbers. The same trend has been observed for both forced (Donzis \& Jagannathan 2013) and decaying (Sciacovelli et al. 2016a) highly compressible homogeneous turbulence. As discussed in Gerolymos \& Vallet (2014), this behaviour is a direct consequence of the perfect-gas equation of state, and does not depend on the specific flow configuration under investigation. Indeed, writing the differential form of the ideal-gas law $(\mathrm{d} p / p)=$ $(\mathrm{d} \rho / \rho)+(\mathrm{d} T / T)$ and approximating differential terms by r.m.s. fluctuations, we get:

$$
\frac{p_{r m s}}{\bar{p}} \approx \frac{\rho_{r m s}}{\bar{\rho}}+\frac{T_{r m s}}{\bar{T}} .
$$

After an initial growth in the linear region, $\rho_{r m s} / \bar{\rho}$ and $T_{r m s} / \bar{T}$ reach a peak in the buffer layer at $y^{*} \approx 10$ and then decay. The pressure fluctuations are rather nearly constant close to the walls, slightly peak at the beginning of the logarithmic zone $\left(y^{*} \approx 40\right)$ and finally decrease, reaching a minimum in the core region. Since pressure fluctuations are of smaller order whereas $\rho_{r m s} / \bar{\rho}$ and $T_{r m s} / \bar{T}$ have very similar distributions and levels, equation (4.5) permits one to identify temperature with density fluctuations, corresponding to the assumption of Laufer (1969) of essentially isobaric 
temperature fluctuations. Some authors (Rubesin 1990; Huang et al. 1995) propose rather that the gas behaves generally in a polytropic manner.

For dense gases, the observed trends are very different. While the overall levels of density and pressure fluctuations remain of the same order of magnitude as for air cases, the relative temperature fluctuations are more than one order of magnitude lower. The fluctuating pressure distribution is much like the perfect-gas one, since it depends on the fluctuating velocity through a Poisson equation. Furthermore, the r.m.s. density decreases monotonically with the wall distance, the highest density fluctuations being observed close to the walls. The r.m.s. density levels decay at higher rates in the linear layer and in the outer region, and exhibit only weak variations in the logarithmic region. This behaviour can also be directly related to the equation of state, namely the Martin-Hou equation (2.10). Following the same reasoning as for the ideal gas, the differential form $d p=(\partial p / \partial \rho)_{T} d \rho+(\partial p / \partial T)_{\rho} \mathrm{d} T$ is derived and the differential terms are approximated by r.m.s. fluctuations, yielding:

$$
\frac{\rho_{r m s}}{\bar{\rho}} \approx \frac{1}{\bar{\rho}}\left[p_{r m s}-\left(\frac{\partial p}{\partial T}\right)_{\rho} T_{r m s}\right] /\left(\frac{\partial p}{\partial \rho}\right)_{T},
$$

where the partial derivatives are evaluated from mean quantities:

$$
\begin{gathered}
\left(\frac{\partial p}{\partial \rho}\right)_{T} \approx \frac{R \bar{T}}{(1-\bar{\rho} b)^{2}}+\sum_{i=2}^{5} \frac{i \bar{\rho}^{i-1} f_{i}(\bar{T})}{(1-\bar{\rho} b)^{i+1}} \\
\left(\frac{\partial p}{\partial T}\right)_{\rho} \approx \frac{\bar{\rho} R}{1-\bar{\rho} b}+\sum_{i=2}^{5} \frac{\bar{\rho}^{i} f_{i}^{\prime}(\bar{p})}{(1-\bar{\rho} b)^{i}} \quad \text { with } f_{i}^{\prime}(\bar{T})=B_{i}-\left(k C_{i} / T_{c r}\right) \exp \left(-k \bar{T} / T_{c r}\right) .
\end{gathered}
$$

The validity of the approximated relation (4.6) is checked in figure 9 for case PM3R7 against DNS results for $\rho_{r m s} / \bar{\rho}$ and appears to be reasonably accurate, especially concerning the observed trends. Then, considering that the thermal variations are weak for the dense-gas temperature fluctuations are neglected, i.e. $T_{r m s}=0$ in (4.6). The corresponding curve follows fairly the trends of $\rho_{r m s} / \bar{\rho}$. The isothermal assumption is then a good approximation for the dense gas, meaning (i) that density fluctuations are correlated with pressure fluctuations and (ii) that $\rho_{r m s} / \bar{\rho}$ is well approximated by $\left(p_{\text {rms }} / \bar{\rho}\right) /(\partial p / \partial \rho)_{T}$.

Finally, using the definition of the sound speed $c^{2}=(\partial p / \partial \rho)_{s}$, and replacing the partial derivative of $p$ with respect to $\rho$ at constant $T$ by the same partial derivative at constant entropy yields $\left(p_{r m s} / \bar{\rho}\right) / \bar{c}^{2}$. The proportionality with the fluid compressibility (inverse of the speed of sound squared) is also tested in figure 9. A good match is obtained above the viscous sublayer, which means that the assumption that the isothermal approximation is also close to an adiabatic approximation for a dense gas is globally true, except very near to the wall. The preceding discussion shows that the rise of density fluctuations near the wall is related to the peculiar behaviour of the thermal equation of state for a nearly isothermal process. Similar results (not shown) were also obtained for a simpler thermal equation of state (Van der Waals) with the same qualitative behaviour, under the polytropic-gas assumption.

Despite the complex variations, fluctuations in thermophysical quantities globally remains moderate for PP11. As a consequence, the assumption of Morkovin (1961), stating that the entropy and vortical modes are weakly coupled, is still valid. 


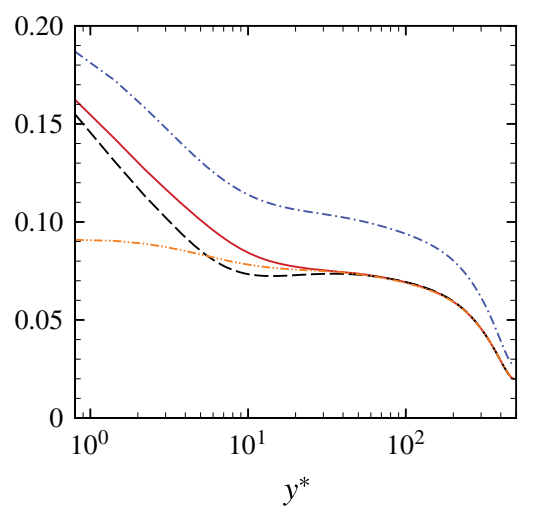

FIgURE 9. (Colour online) Approximation of normalized density fluctuations for case PM3R7. — (red) $\rho_{r m s} / \bar{\rho} ; \ldots \ldots$ (black) $\left[p_{r m s}-(\partial p / \partial T)_{\rho} T_{r m s}\right] /(\partial p / \partial \rho)_{T} / \bar{\rho} ; \ldots \ldots . . .$. (blue) $p_{r m s} /(\partial p / \partial \rho)_{T} / \bar{\rho} ; \cdots \cdots \cdots . .$. (orange) $p_{r m s} / \bar{c}^{2} / \bar{\rho}$.

Fluctuation levels up to $18 \%$ of the mean value are noticed very near the wall for the density, but this region is dominated by viscous stresses, so that the conclusion is not altered.

To further clarify the strong relations between density and pressure, correlation coefficients are calculated. Given two flow variables $\alpha$ and $\beta$, the correlation coefficient $C_{\alpha^{\prime} \beta^{\prime}}$ is defined as:

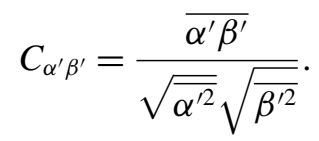

Figure 10 shows the correlations $C_{\rho^{\prime} p^{\prime}}, C_{\rho^{\prime} u^{\prime}}, C_{\rho^{\prime} v^{\prime}}$ and $C_{u^{\prime} v^{\prime}}$. Due to the isothermal boundary conditions, the correlation coefficient $C_{\rho^{\prime} p^{\prime}}$ is equal to unity at the walls for both air and PP11. Afterwards, it decreases reaching a minimum at $y^{*} \approx 8$, approximately corresponding to the temperature variance peak, and then it increases again towards the centreline. For PP11 flows $C_{\rho^{\prime} p^{\prime}}$ approaches unity already at $y^{*} \approx 20$ and then remains constant. On the other hand, it raises slowly for air flows, with an asymptotic value close to 0.9 . The correlation coefficients $C_{\rho^{\prime} u^{\prime}}$ and $C_{\rho^{\prime} v^{\prime}}$, which represent the transport of density fluctuations by the streamwise and wall-normal velocities, exhibit a totally different evolution depending on the fluid nature. Specifically, for air the streamwise correlation is negative and the spanwise is positive, whereas for PP11 both coefficients tend rapidly towards zero. Furthermore, $C_{\rho^{\prime} u^{\prime}}$ exhibits both a Mach and Reynolds dependence for air, whereas for PP11 it varies only with the Mach number. Lastly, the distributions $C_{u^{\prime} v^{\prime}}$ are similar even if a perfect collapse can be noticed for air, whereas a slight Mach dependence is observed for PP11.

Another way to interpret the peculiar behaviour of the thermodynamic quantities is to look at representations in Clapeyron's diagrams. Figure 11 shows distributions of the $p-v$ states in the computational box at one instant for PP11 flows. The different panels correspond successively to the influence of $M_{B}, R e_{B}$ and the wall-normal position. The different $p-v$ diagrams are coloured successively with values of the speed of sound, viscosity and Prandtl number in order to highlight the differences with respect to air. The $p-v$ states are recorded on slices parallel to the wall. The 
(a)

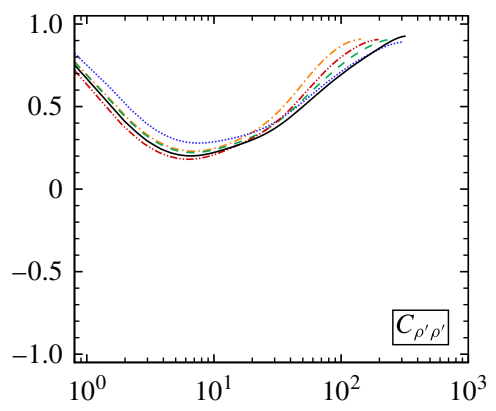

(b)

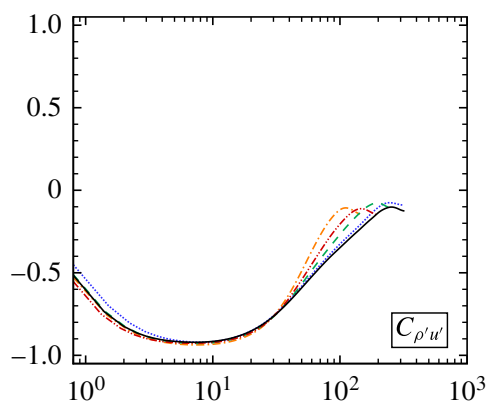

(c)

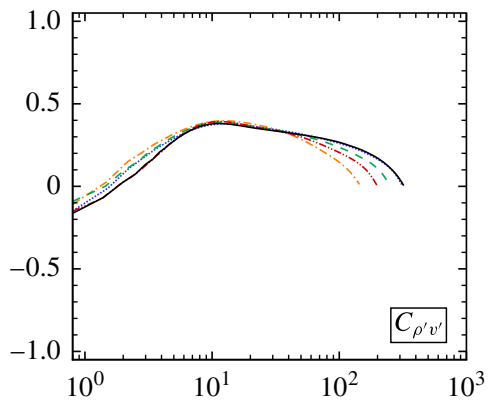

(d)

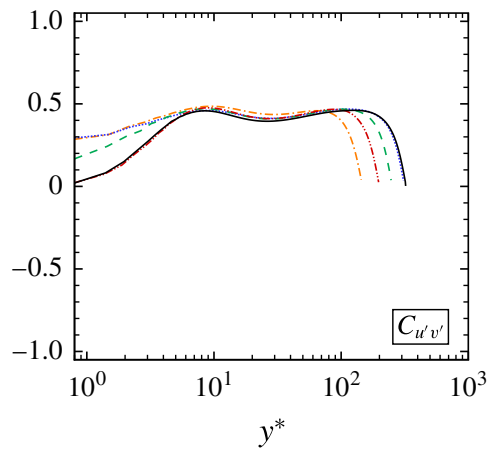

(e)

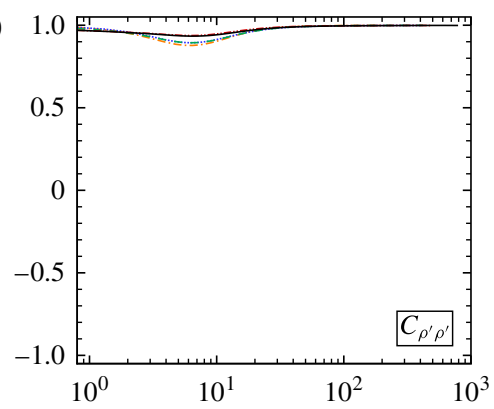

$(f)$

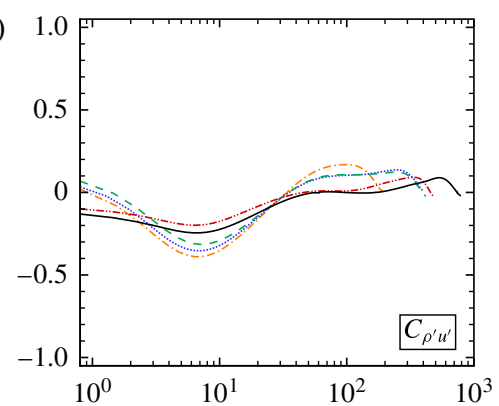

( $g$ )

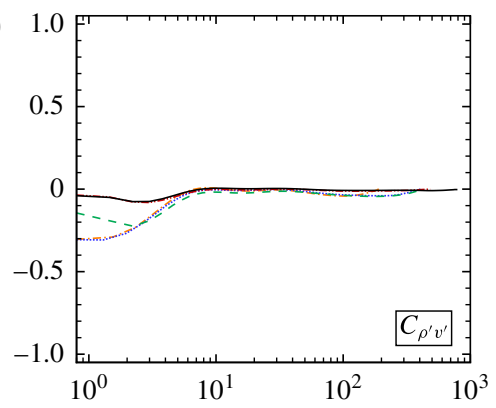

(h)

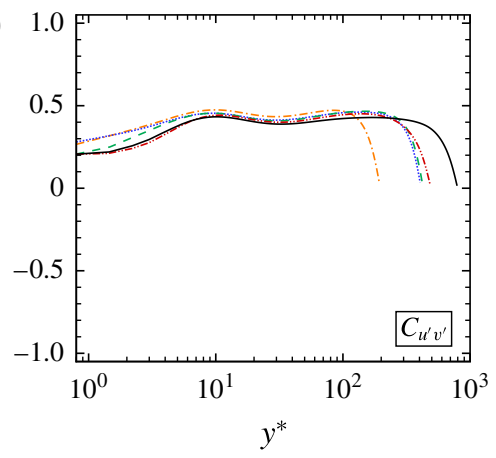

Figure 10. (Colour online) Profiles of the correlation coefficients $C_{\rho^{\prime} p^{\prime}}(a, e), C_{\rho^{\prime} u^{\prime}}(b, f)$, $C_{\rho^{\prime} v^{\prime}}(c, g)$ and $C_{u^{\prime} v^{\prime}}(d, h)$ for air $(a-d)$ and PP11 MAH $(e-h)$ flows.

location is fixed at $y^{*} \approx 10$ where temperature fluctuations peak for figure $11(a, b)$. It is clear from figure $11(a)$ that the main effect of increasing $M_{B}$ is a broadening of the region spanned by the $p-v$ states. For $M_{B}=3$ cases, the states characterized by stronger compressions exit the dense-gas region and reach a supercritical zone 

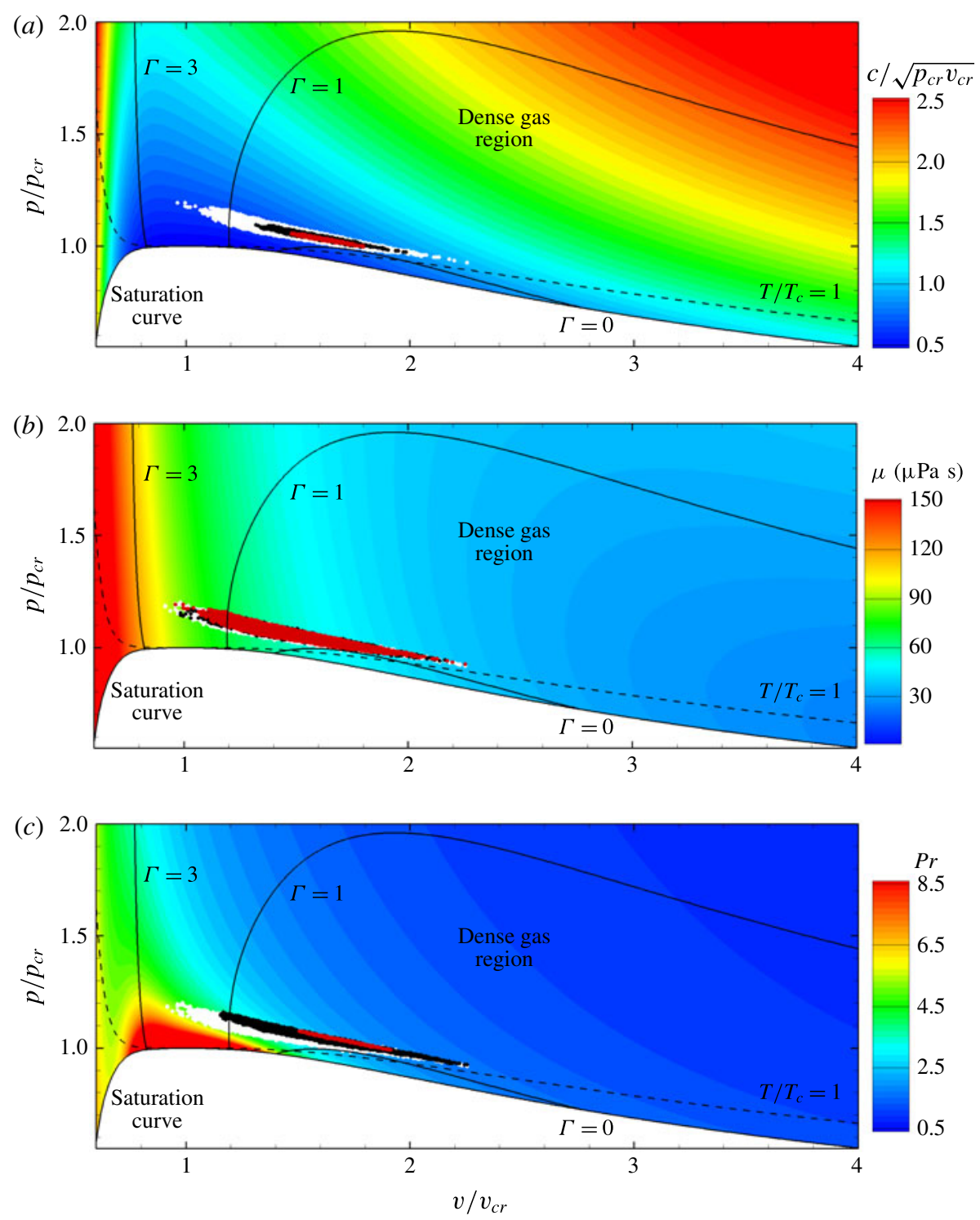

FIGURE 11. (Colour online) Distribution of $p-v$ states of an instantaneous flow field for PP11 in a Clapeyron's diagram. (a) Influence of Mach number on the distribution of $p-v$ states for a slice at $y^{*} \approx 10$ and $\operatorname{Re}_{B}=3000$ : $\left(\bigcirc\right.$, red) $M_{B}=1.5 ;(\bullet) M_{B}=2.25 ;(\bigcirc) M_{B}=3$. The background colour map shows isocontours of the speed of sound. $(b)$ Influence of Reynolds number on the distribution of $p-v$ states for a slice at $y^{*} \approx 10$ and $M_{B}=3$ : $(\bigcirc$, red) $R e_{B}=3000 ;(\bullet) R e_{B}=7000 ;(\bigcirc) R e_{B}=12000$. The background colour map shows isocontours of the viscosity. (c) Influence of $y^{*}$ location on the distribution of $p-v$ states for $M_{B}=3$ and $R e_{B}=12000$ : $\left(\bigcirc\right.$, red) $y^{*}=798$ (centreline); $(\bullet) y^{*}=50$; (○) $y^{*}=10$. The background colour map shows isocontours of the Prandtl number.

where strong variations of the thermodynamic properties are observed. Higher values of $M_{B}$ imply a greater dispersion in $\bar{\rho}_{w}$ values and in variances of the thermodynamic variables near the wall. Changing the Reynolds number $R e_{B}$ for a given $M_{B}$ in figure $11(b)$ reveals that the distributions of $p-v$ states are almost superimposed 
(a)

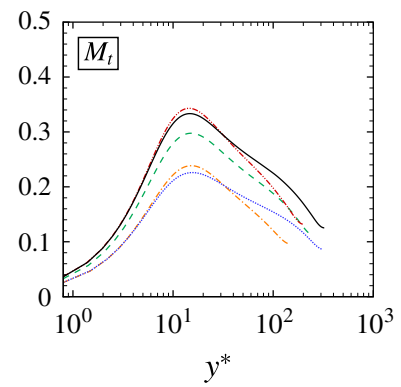

(b)

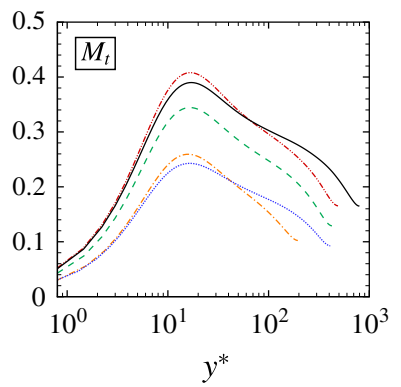

(c)

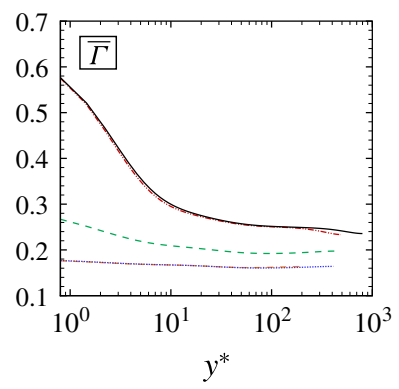

Figure 12. (Colour online) Profiles of the turbulent Mach number $M_{t}$ for air $(a)$ and PP11 (b) and of the fundamental derivative of gas dynamics $\bar{\Gamma}$ for PP11 (c).

for the same $y^{*}$, providing an a posteriori check of the validity of the semi-local scaling. Lastly, distributions for different $y^{*}$ values are shown in figure $11(c)$. The thermodynamic regions spanned by the $p-v$ states are reduced moving away from the wall. Even if most of the states are enclosed in the dense-gas region, none of the present simulations exhibit peculiar dense-gas effects, such as expansion shock waves. This can be explained by the fact that the turbulent Mach number, $M_{t}=\sqrt{u_{i}^{\prime 2}} / \bar{c}$, does not reach sufficiently high values. For instance, figure 12 indicates that $M_{t}$ approaches 0.4 for $M_{B}=3$, whereas Sciacovelli et al. (2016a) have shown that significant dense-gas effects can appear for $M_{t}$ above 0.8 . The distributions of $M_{t}$ are similar as the simulations with air with slightly higher values for PP11 flows, showing that, notwithstanding liquid-like characteristics of dense gases, important compressibility is also present. The profiles of the fundamental derivative of gas dynamics $\bar{\Gamma}$, plotted in figure $12(c)$, confirm that mean values are indeed lower than 1 for PP11 flows, with minimal values in the channel core. The flows thus evolve in the dense-gas region but with positive values of $\bar{\Gamma}$, so that BZT phenomena are not expected.

\section{Influence of dense-gas effects on turbulence structure}

\subsection{Reynolds stresses and Fukagata-Iwamoto-Kasagi (FIK) identity}

Figure 13 shows the Reynolds stresses $\left[\overline{\rho u_{i}^{\prime \prime} u_{j}^{\prime \prime}}\right]^{+}=\overline{\rho u_{i}^{\prime \prime} u_{j}^{\prime \prime}} / \tau_{w}$ for the selected simulations. Patel et al. (2015) have shown that for variable-property turbulent channel flows, gas-like transport properties tend to lower the spanwise, wall-normal and shear Reynolds stresses with respect to the corresponding incompressible evolution, whereas the streamwise component increases. The opposite trend is expected when liquid-like properties are considered. In our cases the strong compressibility adds a complexity in the sense that higher $M_{B}$ increase the velocity gradients near the wall (thus increasing the Reynolds stresses) but also dramatically affect viscosity distributions. Figure 13 provides the trends of Reynolds stresses as a function of both Reynolds and Mach number for selected air and PP11 flows. The figure shows that the Reynolds number dependency is the same for the ideal or dense gas, and is similar to that observed for incompressible flows, even if compressibility effects modify the effective friction Reynolds number for a given bulk Reynolds number. For instance, case AM1R7 $\left(R e_{\tau}^{*} \approx 315\right)$ is characterized by a semi-local friction Reynolds number higher than case AM3R7 $\left(R e_{\tau}^{*} \approx 200\right)$. On the contrary, the dependency on the bulk Mach number is different for the two fluids. In air, the temperature increases with $M_{B}$, and so does 
(a)

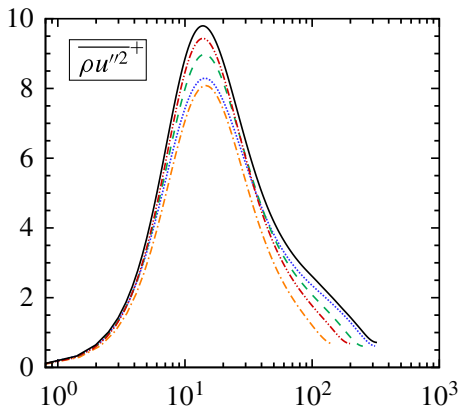

(b)

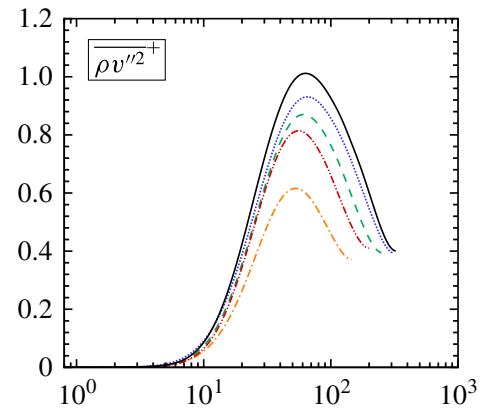

(c)

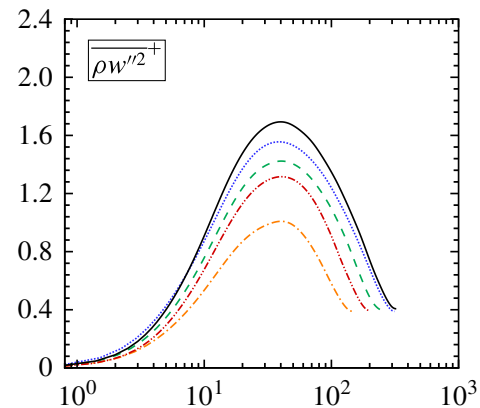

(d)

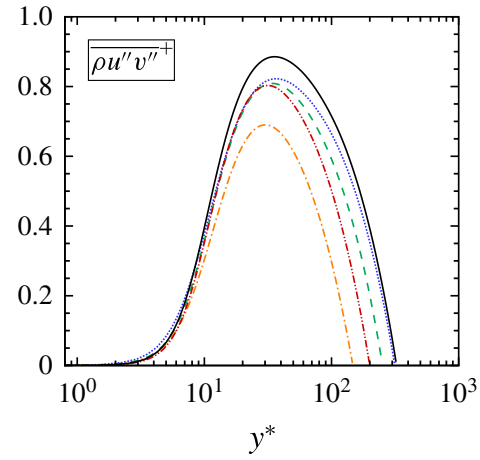

(e)

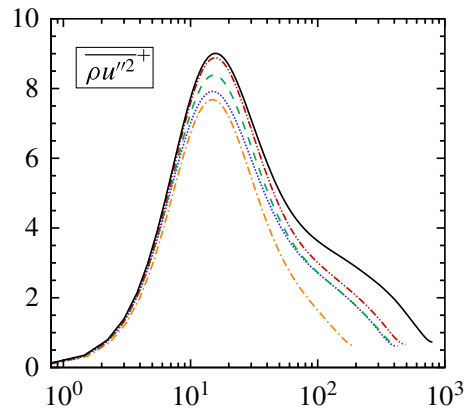

(f)

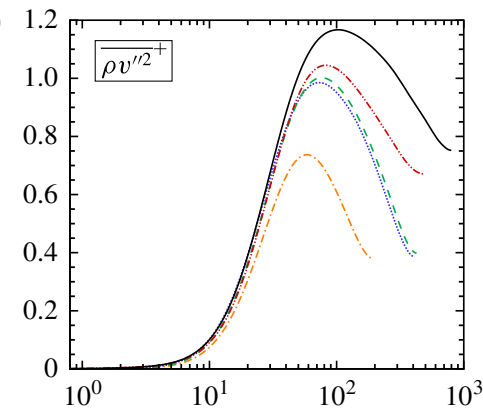

(g)

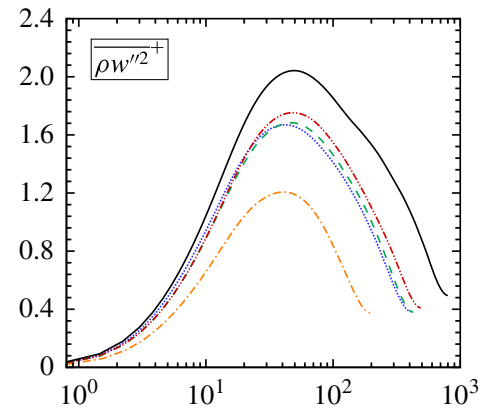

(h)

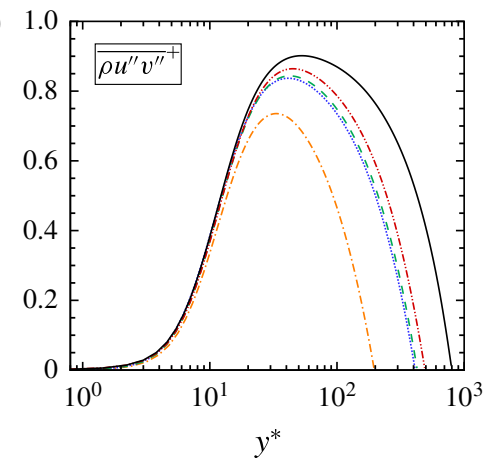

FIgURE 13. (Colour online) Reynolds stresses in the streamwise $(a, e)$, wall-normal $(b, f)$ and spanwise $(c, g)$ directions and Reynolds shear stresses $(d, h)$ for air $(a-d)$ and PP11 $(e-h)$ flows with semi-local scaling.

the viscosity, leading to lower values of $\overline{\rho v^{\prime \prime} v^{\prime \prime}}+\overline{\rho w^{\prime \prime} w^{\prime \prime}}+\overline{\rho u^{\prime \prime} v^{\prime \prime}}+$ as $M_{B}$ grows. For PP11, the viscosity (following the density) becomes lower and lower, thus enhancing 
the spanwise, cross-wise and shear Reynolds stresses. For the streamwise stress, instead, the same behaviour is observed between air and PP11 since the effect of viscosity is now overwhelmed by the increase of the mean velocity shear across the channel.

Concerning the location of the peak values, for both air and PP11 flows they are roughly constant for the streamwise and spanwise components, namely around $y^{*} \in[10,13]$ for ${\overline{\rho u^{\prime 2}}}^{+}$, and $y^{*} \in[40,50]$ for ${\overline{\rho w^{\prime 2}}}^{+}$. On the other hand, a weak dependence on $R e$ is observed for wall-normal and shear stresses. The slight shift towards higher $y^{*}$ is more pronounced for PP11 flows since much higher $R e_{\tau}^{*}$ are achieved. The $R e$ influence is consistent with high Reynolds number incompressible simulations (Lee \& Moser 2015). It can be noticed that wall-normal stresses $\overline{\rho v^{\prime \prime} v^{\prime \prime}}+$ for PP11 flows present different centreline values, notably for cases PM3R7 and PM3R12 at $M_{B}=3$. The levels of wall-normal fluctuations become then of the same level as those in the streamwise direction.

The contribution of Reynolds stresses to the skin friction coefficient can be analysed thanks to the FIK identity. This decomposition of sources of friction also reveals important differences between ideal and dense gases. Starting from the incompressible expression of Fukagata, Iwamoto \& Kasagi (2002), Gomez, Flutet \& Sagaut (2009) derived an exact relationship for the analysis of the contribution of Reynolds stresses to the skin friction coefficient in compressible cases. The skin friction coefficient may be split into four contributions, namely:

$$
\begin{aligned}
& C_{f}^{F I K}=\underbrace{\frac{6}{R e_{B}}}_{C_{L}}+\underbrace{6 \int_{0}^{1}(1-y) \bar{\rho}\left(\widetilde{-u^{\prime \prime} v^{\prime \prime}}\right) \mathrm{d} y}_{C_{T}}+\underbrace{\frac{6}{R e_{B}} \int_{0}^{1}(1-y) \overline{\widehat{\mu}} \frac{\partial \bar{u}}{\partial y} \mathrm{~d} y}_{C_{C}} \\
& +\underbrace{\frac{6}{\operatorname{Re}_{B}} \int_{0}^{1}(1-y) \overline{\mu^{\prime}\left(\frac{\partial u^{\prime}}{\partial y}+\frac{\partial v^{\prime}}{\partial x}\right)} \mathrm{d} y}_{C_{C T}}
\end{aligned}
$$

where $C_{L}$ is the laminar contribution, $C_{T}$ the turbulent contribution, $C_{C}$ the compressible contribution (in which $\overline{\widehat{\mu}}=\bar{\mu}-1$ ) and $C_{C T}$ the compressible-turbulent interaction term. Note that all the quantities in (5.1) are non-dimensionalized using the channel half-width $h$ as length scale, $\widetilde{u}_{B}$ as velocity scale, $\rho_{B}$ as density scale and $\bar{\mu}_{w}$ as viscosity scale. As observed by Fukagata et al. (2002), the turbulent term is proportional to the Favre-averaged Reynolds stresses, and the weighting term decreases linearly with the distance from the wall. This explains why the main contribution to the frictional drag is due to the turbulent structures close to the wall, rather than at the location of the peak of Reynolds stresses. The compressible term $C_{C}$ is related to the mean viscosity variations and to the mean wall-normal velocity gradient. $C_{C T}$ represents the interaction between compressibility and turbulent effects and is usually very small. Table 5 gives the values of $C_{f}^{F I K}$ for different cases, as well as the relative error with respect to the values obtained in $\S 4.1$ and the relative percentage of each contribution. To investigate the effect of compressibility, we consider the cases with $R e_{B}=7000$ and different $M_{B}$, such that the laminar contribution $C_{L}$ is approximately constant (i.e. $C_{L} \approx 13 \%-14 \%$ ). For air flows, the contribution due to the turbulent fluctuations $C_{T}$ decreases as $M_{B}$ increases, coherently with the decreasing of the Reynolds stress levels. The opposite trend is observed for PP11 flows, for which the relative weight of $C_{T}$ increases of approximately $1.5 \%$. This can be related to 


\begin{tabular}{lcrrrrr} 
Case & $C_{f}^{F I K}\left(\times 10^{3}\right)$ & Error $(\%)$ & $C_{L}(\%)$ & $C_{T}(\%)$ & \multicolumn{1}{c}{$C_{C}(\%)$} & $C_{C T}(\%)$ \\
AM1R7 & 6.41 & 0.3 & 13.28 & 84.63 & 2.04 & 0.05 \\
AM2R7 & 6.37 & 1.8 & 13.26 & 82.19 & 4.45 & 0.11 \\
AM3R7 & 6.36 & 3.8 & 13.15 & 79.08 & 7.60 & 0.17 \\
PM1R7 & 6.24 & -0.8 & 13.76 & 86.71 & -0.48 & 0.02 \\
PM2R7 & 6.15 & 0.4 & 14.05 & 87.00 & -1.07 & 0.02 \\
PM3R7 & 6.04 & 0.9 & 14.31 & 88.21 & -2.55 & 0.02
\end{tabular}

TABLE 5. Contributions to the skin friction coefficient according to the compressible extension of the FIK identity of Gomez et al. (2009) for different cases.

the liquid-like behaviour of dense gases, which also causes substantial changes in the compressible contribution $C_{C}$. In fact this term takes on higher values as $M_{B}$ increases for both fluids, but it is positive for air (since $\bar{\mu}>0$ ) and negative for PP11 $(\overline{\widehat{\mu}}<0)$. The compressible term is indeed related to variable-property effects. Lastly, the compressible-turbulent interaction term is always quite small and contributes to the total skin friction coefficient for approximately $0.1 \%$ in air and $0.02 \%$ in PP11 flows, for which the dynamic and thermal effects are even less correlated.

\subsection{Turbulent kinetic energy budgets}

For fully developed, statistically stationary channel flow, the turbulent kinetic energy budget reads $P_{k}+T_{k}+V_{k}+M_{k}+D_{k}+\varepsilon_{k}=0$, with

$$
\begin{aligned}
& P_{k}=-\bar{\rho} \widetilde{u^{\prime \prime} v^{\prime \prime}} \frac{\mathrm{d} \tilde{u}}{\mathrm{~d} y} \\
& T_{k}=-\frac{\mathrm{d}}{\mathrm{d} y} \frac{1}{2}\left(\overline{\rho u^{\prime \prime 2} v^{\prime \prime}}+\overline{\rho v^{\prime \prime 3}}+\overline{\rho w^{\prime 2} v^{\prime \prime}}\right) \\
& V_{k}=\frac{\mathrm{d}}{\mathrm{d} y}\left(\overline{u^{\prime} \tau_{x y}^{\prime}}+\overline{v^{\prime} \tau_{y y}^{\prime}}+\overline{w^{\prime} \tau_{y z}^{\prime}}\right) \\
& M_{k}=\overline{u^{\prime \prime}}\left(-\frac{\partial \bar{p}}{\partial x}+\frac{\mathrm{d} \overline{\tau_{x y}}}{\mathrm{~d} y}\right)+\overline{v^{\prime \prime}}\left(-\frac{\partial \bar{p}}{\partial y}+\frac{\mathrm{d} \overline{\tau_{y y}}}{\mathrm{~d} y}\right)+\overline{w^{\prime \prime}} \frac{\mathrm{d} \overline{\tau_{y z}}}{\mathrm{~d} y} \\
& D_{k}=\overline{p^{\prime} \frac{\partial u^{\prime}}{\partial x}}+\overline{p^{\prime} \frac{\partial v^{\prime}}{\partial y}}-\frac{\mathrm{d}}{\mathrm{d} y} \overline{v^{\prime} p^{\prime}}+\overline{p^{\prime} \frac{\partial w^{\prime}}{\partial z}} \\
& \left.\varepsilon_{k}=-\overline{\left(\tau_{x j}^{\prime} \frac{\partial u^{\prime}}{\partial x_{j}}\right.}+\overline{\tau_{y j}^{\prime} \frac{\partial v^{\prime}}{\partial x_{j}}}+\overline{\tau_{z j}^{\prime} \frac{\partial w^{\prime}}{\partial x_{j}}}\right)
\end{aligned}
$$

$P_{k}$ is the production, $T_{k}$ and $V_{k}$ the turbulent and viscous transport respectively, $M_{k}$ the mass flux variation, $D_{k}$ the velocity-pressure gradient tensor (composed of the sum of pressure strain correlation and pressure transport term) and $\varepsilon_{k}$ the dissipation term. Figure 14 shows the turbulent kinetic energy budget for cases AM3R12 and PM3R12 normalized by $\tau_{w}^{2} / \bar{\mu}(y)$ (Foysi et al. 2004). In semi-local scaling, the viscous and turbulent transports behave similarly, whereas the mass flux variation (which is zero for incompressible flows) is much smaller for PP11 case. In the core region, all the budget terms collapse.

A closer inspection of the turbulent production and dissipation for the selected simulations is provided in figure 15, which also shows their ratio, plotted both in 


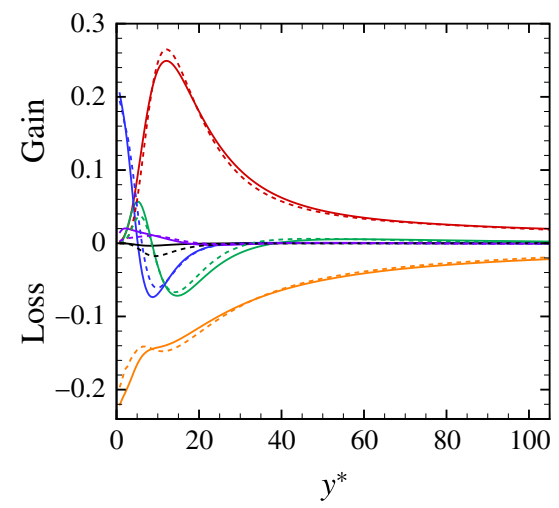

FIGURE 14. (Colour online) Comparison of turbulent kinetic energy budgets for cases AM3R12 (dashed lines) and PM3R12 (solid lines) in semi-local scaling: - (red) $P_{k}$, - (green) $T_{k}$, - (blue) $V_{k},-$ (black) $M_{k},-$ (purple) $D_{k},-$ (orange) $\varepsilon_{k}$ normalized by $\tau_{w}^{2} / \bar{\mu}(y)$.
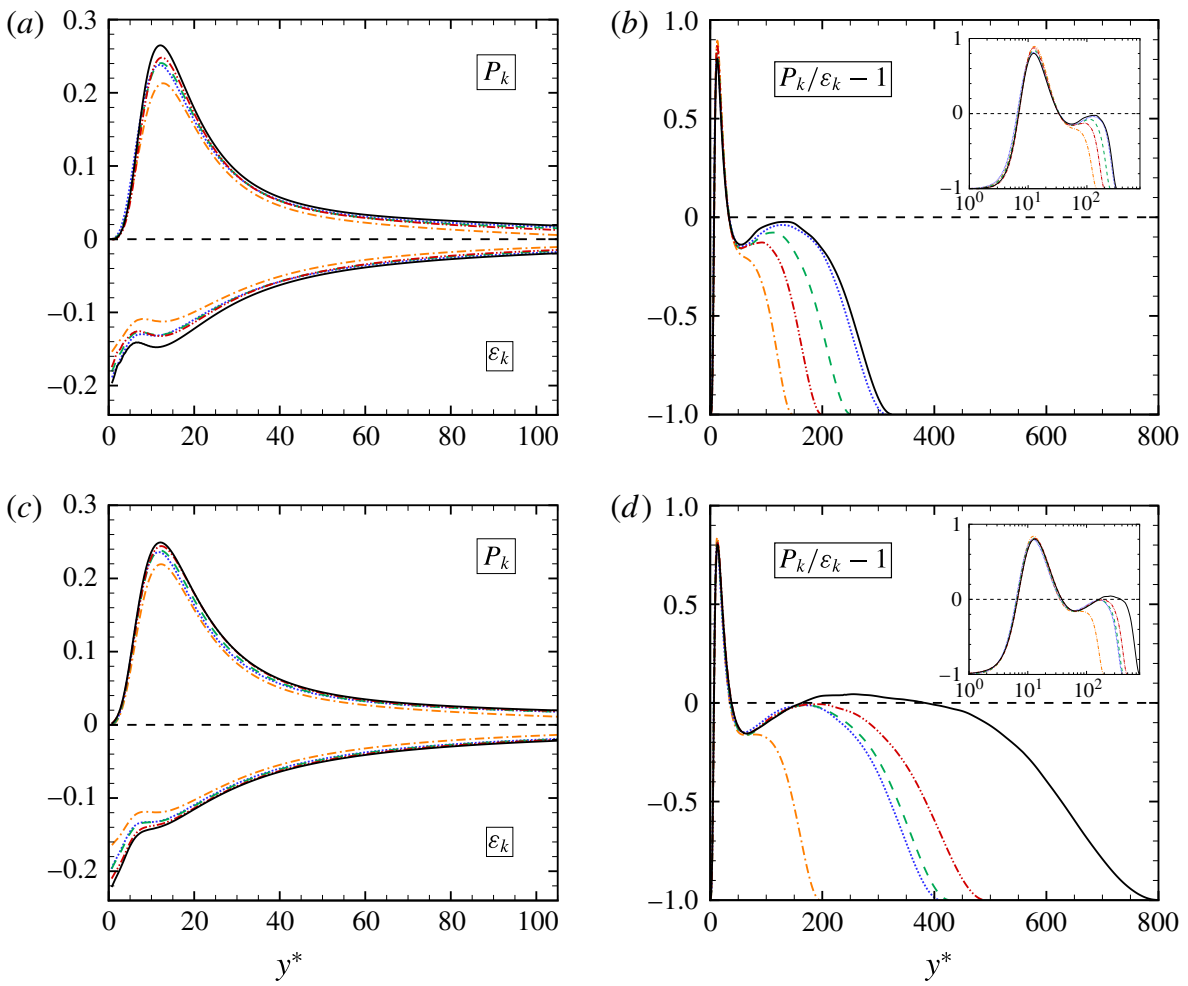

FIGURE 15. (Colour online) Profiles of the turbulent production $P_{k}$, the turbulent dissipation $\varepsilon_{k}$ and the ratio of production to dissipation $P_{k} / \varepsilon_{k}-1$ for air $(a, b)$ and PP11 $(c, d)$ flows.

linear and logarithmic semi-local coordinates. For both fluids, the production peak is located at $y^{*} \approx 11-12$ for all the considered bulk conditions. Sarkar (1995) and Lechner et al. (2001) showed that the main effect of compressibility is a reduction 
(a)
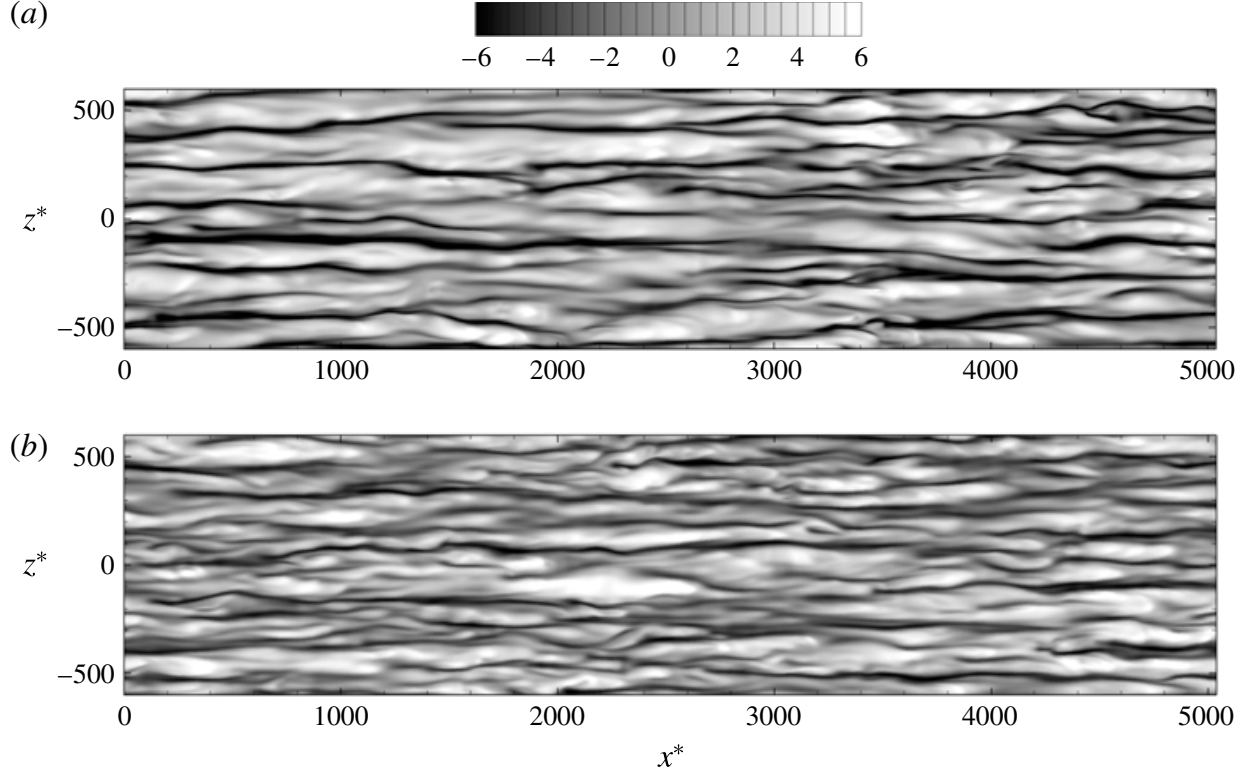

FIGURE 16. $\sqrt{\rho} u^{\prime \prime} / \sqrt{\tau_{w}}$ fluctuations at $y^{*} \approx 12$ for $\operatorname{AM} 3 R 7(a)$ and PM1R3 $(b)$.

in the production and dissipation rate of the turbulent kinetic energy. Nevertheless, this effect can be associated with the strong variations of properties at high Mach numbers. The semi-local scaling $\tau_{w}^{2} / \bar{\mu}(y)$ is able to collapse, for instance, the budgets for cases M1R7, M2R7 and M3R7, for which substantial differences should be observed due to the doubling of the bulk Mach number. Globally, a common trend is observed for air and PP11 flows, where the peaks of production and dissipation terms slightly increase with $R e_{\tau}^{*}$. The ratio of the production and dissipation rates $P_{k} / \varepsilon_{k}-1$ exhibits a similar evolution for similar values of $R e_{\tau}^{*}$ (cases PM1R3 and AM3R7). The ratio peaks at the same $y^{*}$ location as the production term. Furthermore, for cases with $R e_{\tau}^{*}>200$, a second peak starts growing in the logarithmic region. For PP11 flows, the inner peak seems roughly unaffected, as found for high Reynolds number turbulent incompressible flows (Lee \& Moser 2015), whereas in air a slight decrease is observed with increasing $R e_{\tau}^{*}$, as shown in the inset with logarithmic scale. Since the $R e_{\tau}^{*}$ values are low in air cases, the ratio always remains negative and no effective net turbulent production is observed in the outer layer. For PP11, the case PM3R12 is the only one with a positive region, extending between $y^{*} \approx 180$ and $y^{*} \approx 400$.

\subsection{Near-wall turbulent structures}

The difference in near-wall turbulent structures is now analysed by means of instantaneous flow visualizations for iso- $R e_{\tau}^{*}$ cases, namely, AM3R7 and PM1R3 $\left(R e_{\tau}^{*} \approx 200\right.$ at the centreline). Figures $16-18$ show a slice of the instantaneous flow field at different values of $y^{*}$ (namely, $y^{*}=12,50$ and centreline) coloured with levels of the normalized streamwise velocity fluctuations $\sqrt{\rho} u^{\prime \prime} / \sqrt{\tau_{w}}$ for cases AM3R7 and PM1R3 (hence for $R e_{\tau}^{*} \approx 200$ ). The local density value $\rho$ is used for normalization instead of $\bar{\rho}$, since it correlates better with the modulation of the streak magnitude (Patel et al. 2015). In all cases, the computational box is scaled with respect to semi-local coordinates $\left(x^{*}, z^{*}\right)$ (which change with the wall distance) and the box 

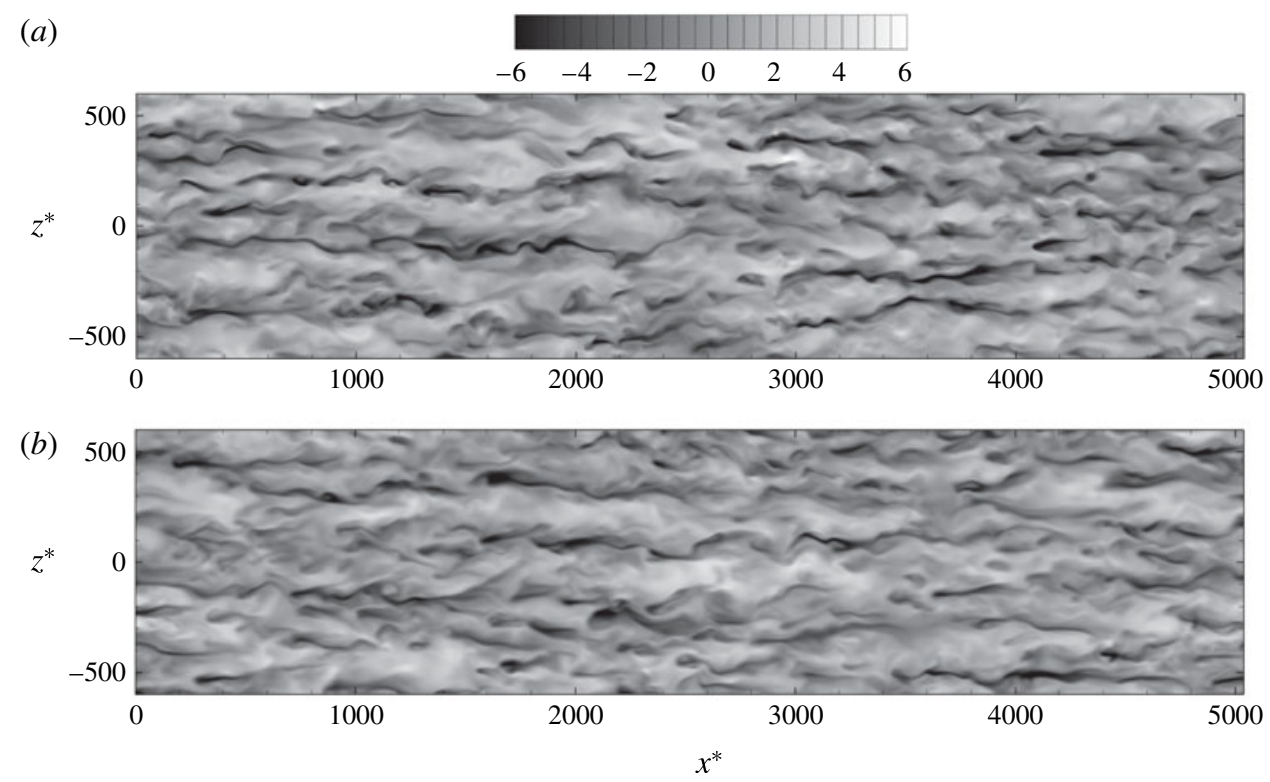

FIGURE 17. $\sqrt{\rho} u^{\prime \prime} / \sqrt{\tau_{w}}$ fluctuations at $y^{*} \approx 50$ for $\operatorname{AM} 3 R 7(a)$ and PM1R3 $(b)$.

(a)
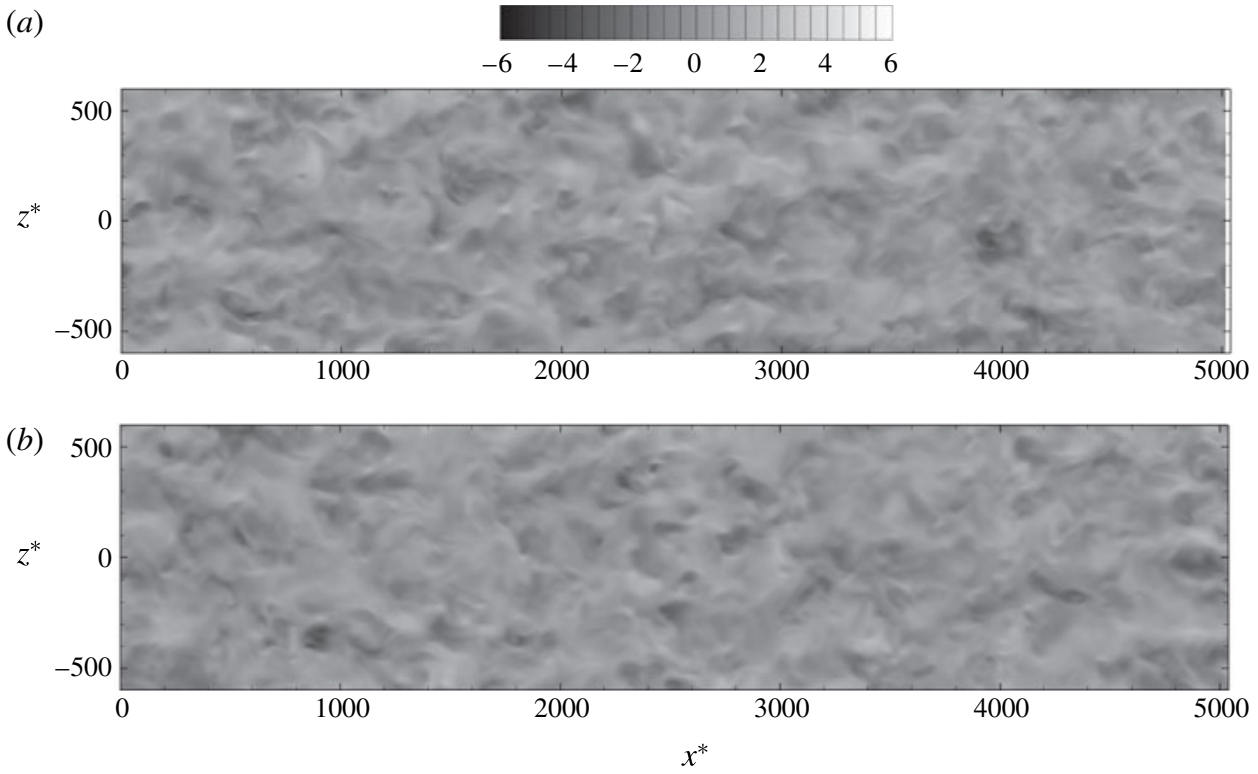

FIGURE 18. $\sqrt{\rho} u^{\prime \prime} / \sqrt{\tau_{w}}$ fluctuations at $y^{*} \approx 200$ for $\operatorname{AM} 3 R 7(a)$ and PM1R3 $(b)$.

size considered is $5000 \times 1200$ based on non-dimensional coordinates $x^{*} \times z^{*}$ for each case. For both fluids, the maps of the instantaneous streamwise velocity close to the wall exhibits the well-known organization in streaks (Coleman et al. 1995; Chernyshenko \& Baig 2005; Gerolymos \& Vallet 2014). In past years, the role of compressibility on the modification of near-wall structures has been widely discussed. Initially, it was argued that its effect was to increase the streamwise correlation length, enhancing the coherence of near-wall streaks (Coleman et al. 1995; Duan et al. 2010; 
(a)
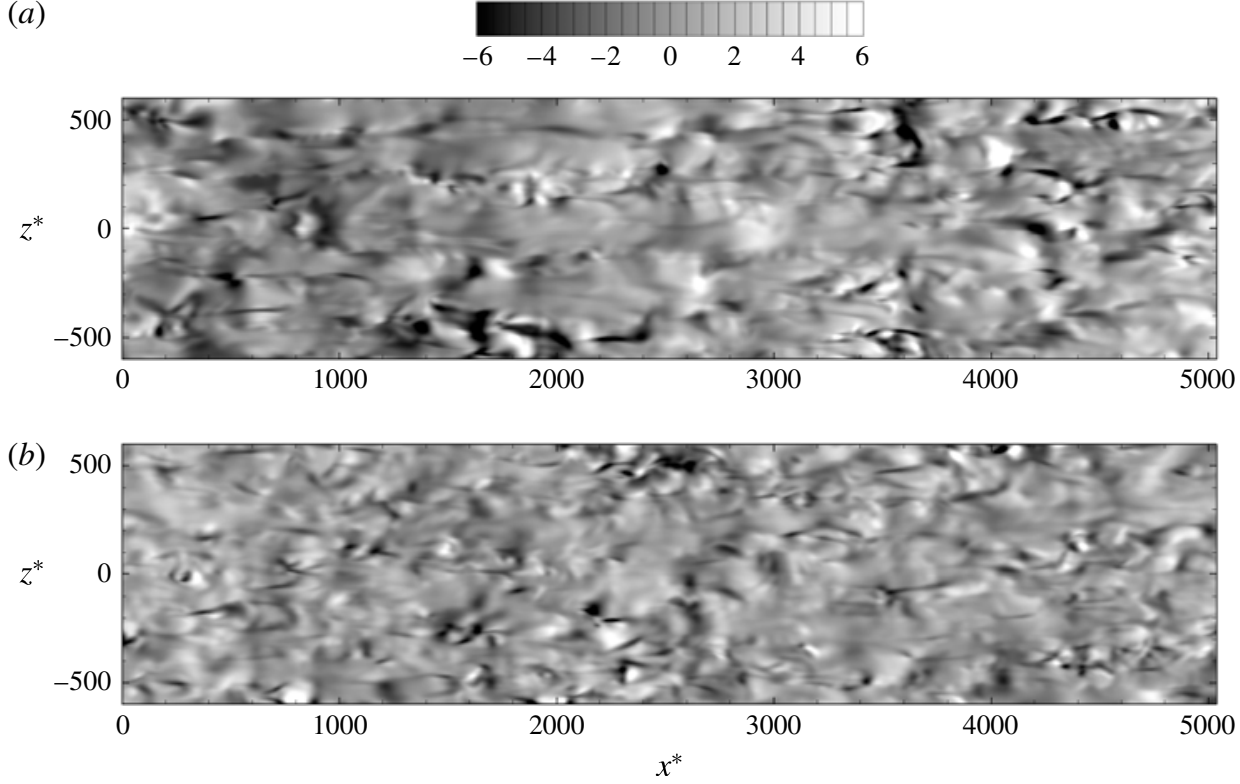

FIgURE 19. Pressure fluctuations $p^{\prime+}$ at $y^{*} \approx 12$ for $\operatorname{AM} 3 R 7(a)$ and PM1R3 $(b)$.

Lagha et al. 2011). This statement was not confirmed when the semi-local scaling was taken into account (Morinishi et al. 2004; Patel et al. 2015). A rather universal behaviour was then observed. The latter conclusion holds in the present comparison between air and PP11 flows at the three heights shown in the figures. Figure 16 shows that near-wall layers are dominated by streaks with similar streamwise lengths and spanwise separations. At $y^{*} \approx 50$, in figure 17 , the structures become less coherent and less energetic. At the centreline of the channel, as illustrated in figure 18, no prominent coherent structures can be found.

Differently from velocity fields which have a similar structural behaviour if represented with the semi-local scaling, the thermodynamic quantities are much more sensitive. Figures 19 and 20 show the instantaneous pressure $p^{\prime+}$ and density $\rho^{\prime+}$ fluctuations at $y^{*} \approx 12$. Pressure fluctuations are characterized in both cases by long-range interactions (Kim 1989; Chang III, Piomelli \& Blake 1999), even if the mean square fluctuations mainly have local contributions (Kim 1989). Higher deviations from the mean value are observed in air. Contrary to pressure, density fluctuations behave differently between air and PP11 flows. For air, very elongated scales in the streamwise direction are preferentially observed and follow approximately velocity fluctuations. In fact, the correlation $C_{\rho^{\prime}, u^{\prime}}$ is close to -1 in the buffer layer. Consequently, negative (positive) density fluctuations are associated with positive (negative) velocity fluctuations. Low-speed streaks are thus characterized by higher-density fluid and vice versa. The behaviour for PP11 flows is rather different, as inferred from the correlation coefficients $C_{\rho^{\prime}, u^{\prime}}$ and $C_{\rho^{\prime}, p^{\prime}}$ in figure 10 . $C_{\rho^{\prime}, u^{\prime}}$ being much smaller in the buffer layer, the density modulation for low and high-speed streaks is less marked. Instead, the pressure signature is visible on the instantaneous density since $C_{\rho^{\prime}, p^{\prime}} \approx 1$ across the whole channel height.

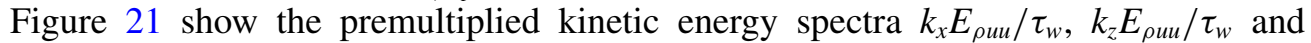
$k_{z} E_{\rho v v} / \tau_{w}$ for cases AM3R7 and PM1R3 on the whole channel height as a function 
(a)
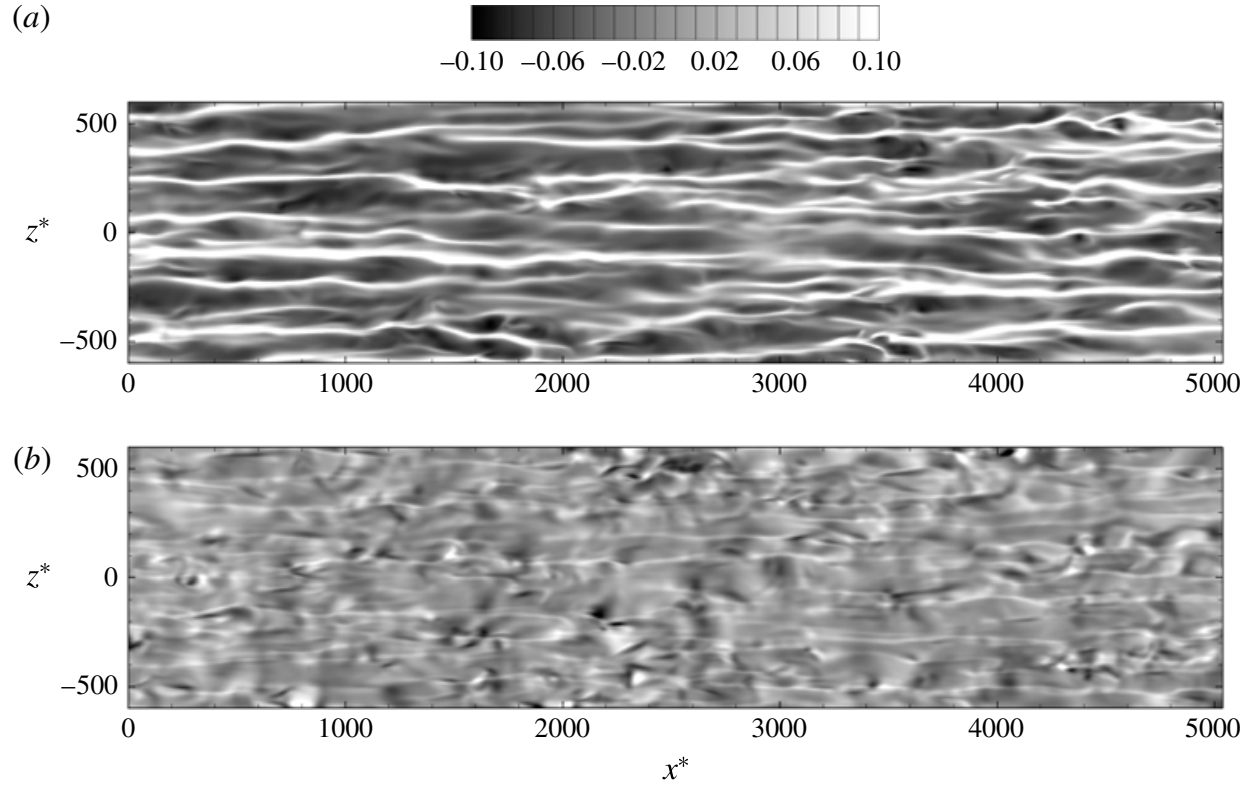

FIGURE 20. Density fluctuations $\rho^{+}$at $y^{*} \approx 12$ for $\operatorname{AM} 3 R 7(a)$ and PM1R3 $(b)$.

(a)

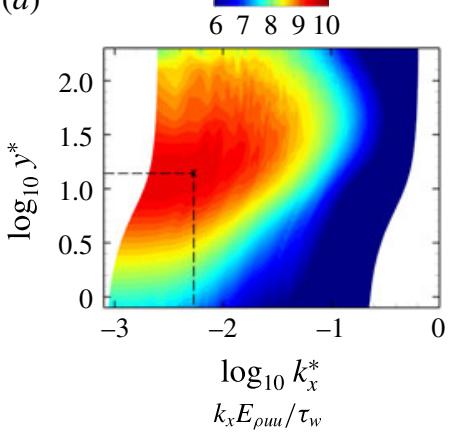

$(d)$

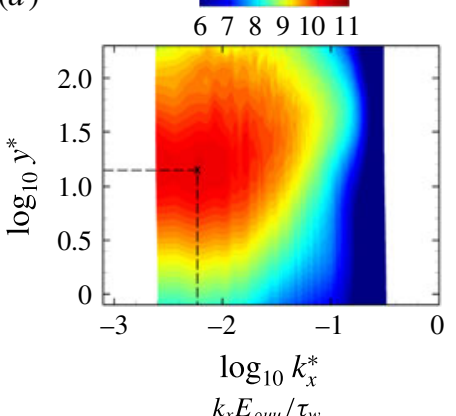

(b)

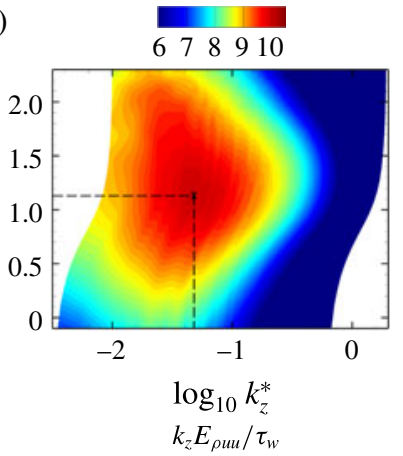

(e)

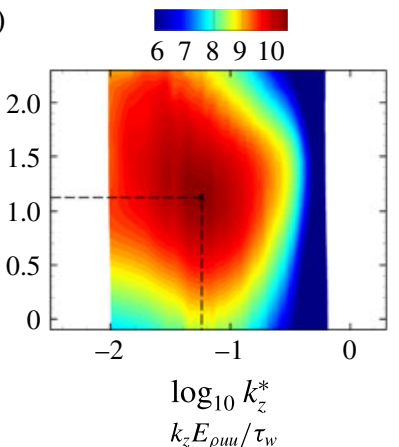

(c)

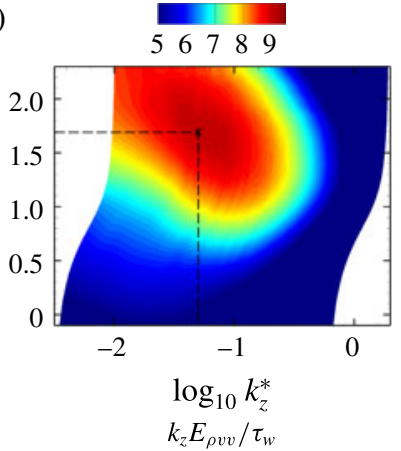

(f)

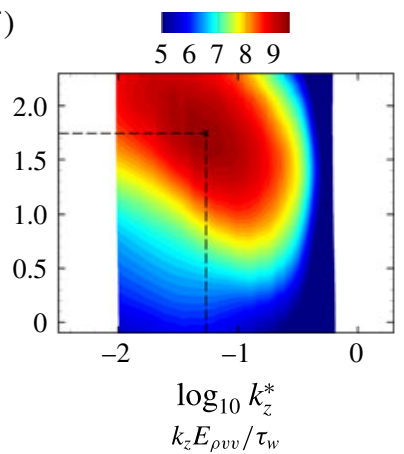

FIgURE 21. (Colour online) Premultiplied energy spectra for cases AM3R7 $(a)$ and PM1R3 $(b)$. 
(a)

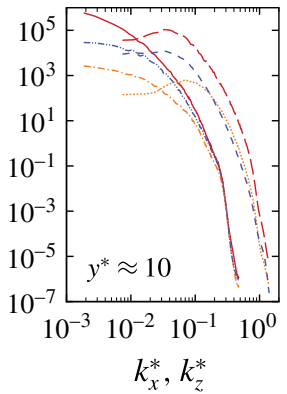

(b)

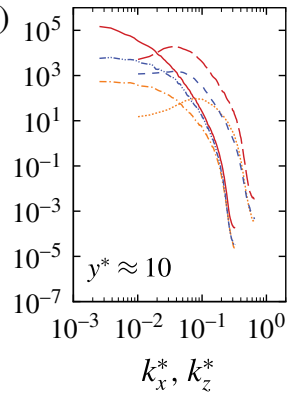

(c)

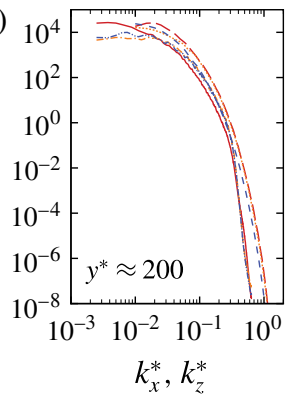

(d)

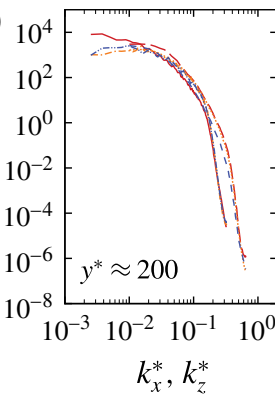

FIGURE 22. (Colour online) One-dimensional spectra for cases AM3R7 $(a, c)$ and PM1R3

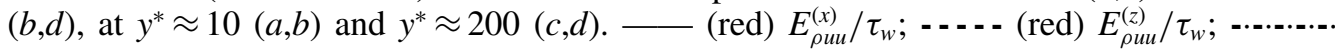
(orange) $E_{\rho v v}^{(x)} / \tau_{w} ; \cdots \cdots$ (orange) $E_{\rho v v}^{(z)} / \tau_{w} ; \cdots \cdots \cdots$ (blue) $E_{\rho w w}^{(x)} / \tau_{w} ; \cdots \cdots$ (blue) $E_{\rho w w}^{(z)} / \tau_{w}$.

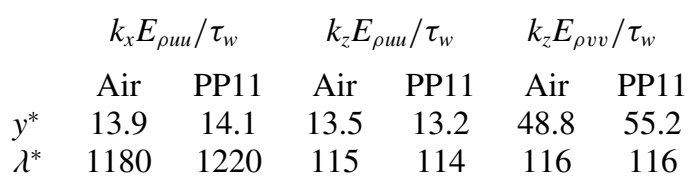

TABLE 6. Values of $y^{*}$ and $\lambda^{*}$ for the peak values of the spectra shown in figure 21 .

of semi-local streamwise $k_{x}^{*}$ and spanwise $k_{z}^{*}$ wavenumbers. The semi-local scaling provides an approximate collapse over a wide range of wavenumbers, improving substantially the classical inner scaling. The strongest modifications are obtained for air flows, in which the high gradients of variable properties near the wall alter the effective scaled wavenumbers up to the buffer layer. This corresponds to elongated (shortened) structures for gas-like (liquid-like) transport properties with respect to the incompressible regime (Coleman et al. 1995; Duan et al. 2010). The locations $y^{*}$ and the wavelengths $\lambda^{*}$ corresponding to the peak values are reported in table 6. Similar values with respect to those typically obtained in incompressible turbulence are found for both fluids. For instance, for the streamwise velocity, an inner peak is detected at $y^{+} \approx 15$, where $\lambda_{x}^{+} \approx 1000$ and $\lambda_{z}^{+} \approx 100$ (see, e.g. spectra databases of Kim et al. (1987) and Moser, Kim \& Mansour (1999)). One-dimensional spectra for the three velocity components, given in figure 22, show that the spanwise length scale for the wall-normal component is approximately half of the one associated with $u$ at the same distance from the wall. Table 6 reports the global maximum non-dimensional wavenumber which arises at different wall distances $\left(y^{*} \approx 13-50\right.$ respectively).

Near-wall turbulent structures can be also analysed by means of the quadrant analysis (Wallace 2016). This representation allows a detailed study of the physical mechanisms producing the Reynolds shear stresses and, in a related way, underlying the turbulent kinetic energy production. The $(u, v)$ plane is divided into four quadrants: Q1 $\left(+u^{\prime \prime},+v^{\prime \prime}\right), \mathrm{Q} 2\left(-u^{\prime \prime},+v^{\prime \prime}\right), \mathrm{Q} 3\left(-u^{\prime \prime},-v^{\prime \prime}\right), \mathrm{Q} 4\left(+u^{\prime \prime},-v^{\prime \prime}\right)$. The contribution of the different quadrants can be obtained by plotting the isocontours of the probability-weighted Reynolds shear stresses $\rho u^{\prime \prime} v^{\prime \prime} / \tau_{w} P\left(\sqrt{\rho} u^{\prime \prime} / \sqrt{\tau_{w}}, \sqrt{\rho} v^{\prime \prime} / \sqrt{\tau_{w}}\right)$, 


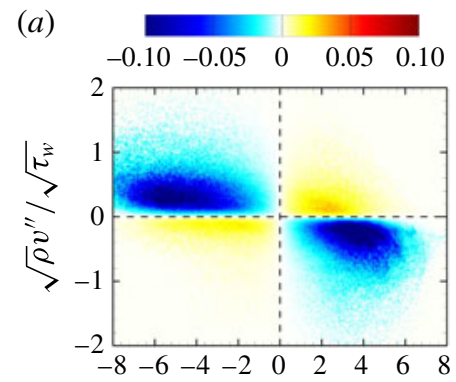

(b)

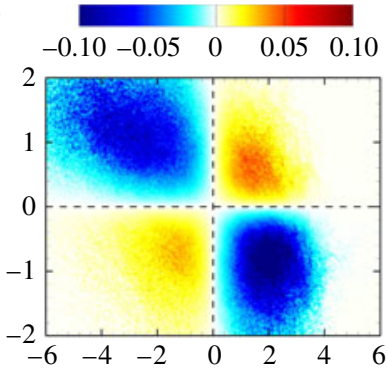

(c)

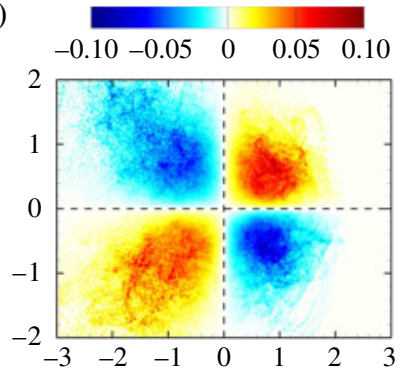

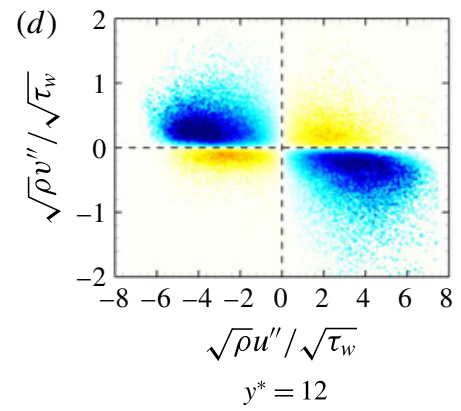
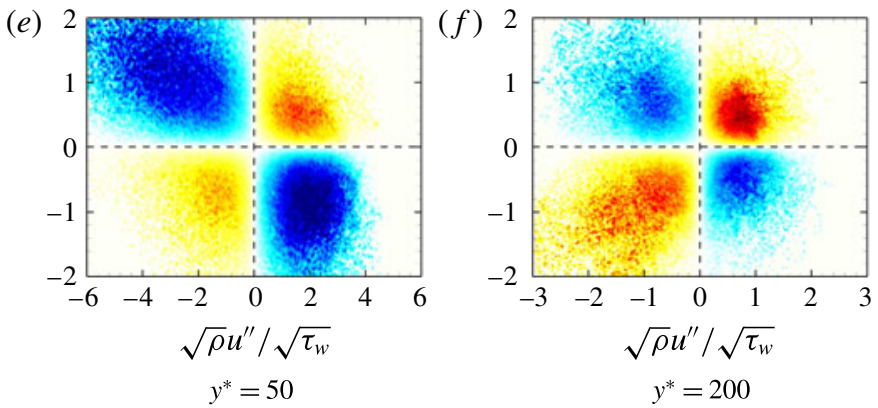

FIGURE 23. (Colour online) Probability-weighted Reynolds shear stress $\rho u^{\prime \prime} v^{\prime \prime} / \tau_{w} P\left(\sqrt{\rho} u^{\prime \prime} / \sqrt{\tau_{w}}, \sqrt{\rho} v^{\prime \prime} / \sqrt{\tau_{w}}\right)$ for air $(a-c)$ and PP11 $(d-f)$.

depicted in figure 23. Near the walls probability-weighted Reynolds shear stresses appear stretched in the streamwise direction and flatter in the spanwise direction, with a strong contribution in the Q2 and Q4 quadrants, corresponding respectively to ejections $\left(u^{\prime \prime}<0, v^{\prime \prime}>0\right)$ and sweeps $\left(u^{\prime \prime}>0, v^{\prime \prime}<0\right)$. Moving towards the centreline, the contributions become more isotropic. The most important differences between air and PP11 are found in the buffer layer. Namely, the contribution of ejections events to shear stresses appear lower for PP11 flows with respect to air flows. This is consistent with recent findings of Patel et al. (2015). Gas-like (liquid-like) transport properties tend to stabilize (weaken) the low-speed streaks, which lift less (more) intensely away from the wall.

\section{Conclusions}

Direct numerical simulations of supersonic turbulent channel flow of dense gases were carried out by selecting a heavy fluorocarbon, called PP11, as representative of the dense-gas behaviour in the saturated vapour region. This fluid is characterized by an extended range of thermodynamic conditions in the vapour phase such that the fundamental derivative of gas dynamics, $\Gamma$, is lower than one; additionally, this fluid is predicted to exhibit a relatively wide inversion zone. Both features lead to a non-monotonic variation of the speed of sound with density in isentropic perturbations. Another striking point is that, in the dense-gas region, the fluid transport properties depend both on temperature and density. Dense-gas effects on wall turbulence are investigated by means of a parametric study at three bulk Reynolds numbers (namely, 
$R e_{B}=3000,7000$ and 12000) and three bulk Mach numbers $\left(M_{B}=1.5,2.25\right.$ and 3). The thermodynamic behaviour of PP11 is well represented by the Martin-Hou thermal equation of state. For comparison, compressible DNS with the same parameters are also carried out for air flows, under the ideal-gas assumption. The main results are as follows.

The analysis of mean and fluctuating thermodynamic and transport properties reveals several peculiarities of wall-bounded dense-gas flows. Primarily, unlike perfect gases where viscous heating leads to high centreline temperatures, in dense-gas flows the high specific heat leads to negligible temperature variations, thus reducing the need for wall cooling. As a consequence the wall behaves as isothermal and (nearly) adiabatic at the same time. Since the temperature is almost constant throughout the flow, variations of the thermodynamic and transport properties are tightly correlated to the fluid density (or pressure). Specifically, speed of sound and viscosity variations are essentially due to the density, which decreases from the walls toward the centreline. On the one hand, this leads to less marked increase of speed of sound at the channel centreline and, subsequently, to centreline Mach numbers greater than the bulk ones, contrary to perfect gases. On the other hand, in the dense-gas region viscosity has an opposite behaviour with respect to the density, and decreases toward the channel centre. As a consequence, compressible dense-gas flows are found to share many features found in variable-property liquids, albeit the driving mechanisms are deeply different (in liquids viscosity decreases due to temperature increasing toward the centreline). This is confirmed by global quantities such as the friction coefficient or the Nusselt number, which are important for industrial purposes. Specifically, the former is well described by a compressible extension of the FIK identity accounting for fluid property variations and follows reasonably well a classical empirical correlation for incompressible flows, unlike perfect gases with exhibit significant Mach number effects. Despite the apparent liquid-like behaviour, supersonic flows of dense gas are characterized by strong compressibility effects. This other facet is highlighted by the distributions of the turbulent Mach number which are similar as those observed for a perfect gas but with slightly higher levels, due to the overall lower speed of sound. Note that, for this confined flows, the maximum values obtained with the considered range of bulk Mach numbers remain below the limit where strong compressibility effects, like eddy shocklets, are visible. Peculiar features of dense gas, such as BZT phenomena where expansion shocks become admissible, are thus not observed. The study of the local value of the derivative of gas dynamics $\Gamma$, which is an indicator of dense-gas effects, indeed showed that the fluid evolves in the dense-gas region $(\Gamma<1)$ without taking on negative values, where BZT phenomena can occur. Even without BZT effects, the peculiar behaviour of the speed of sound in dense gases deeply modifies fluctuating density profiles leading to, e.g. maximum r.m.s. levels located close to the channel wall instead of in the buffer layer as in standard compressible channel flows.

This dual behaviour that bears both liquid-like and gas-like features helps to shed some light on the modification of wall turbulence in the presence of a dense gas. As already shown in previous studies for both supersonic channel flows or low-speed variable-property channel flows, the semi-local scaling introduced by Huang et al. (1995), and later formalized by Patel et al. (2015) or Trettel \& Larsson (2016), is well adapted to compare results from existing surveys and with the well-documented incompressible limit. Note that the Van Driest transformation already provides a good collapse for PP11 flows since the heat transfer at the walls is weak. The comparison of second-order statistics and instantaneous views of the velocity fields 
also gives a strong support for the validity of semi-local scaling with dense-gas flows. Overall, this amounts to saying that Morkovin's hypothesis is still a good framework for this complex fluid. The fact that the order of magnitude of density and pressure fluctuations is roughly similar to air flows and that a strong reduction of temperature fluctuations is noticed reinforces this conclusion. The structure of turbulence is thus not deeply altered in dense-gas supersonic channel flows. The main effect of variable properties can be taken into account by using the semi-local scaling, revealing the weak coupling between the vortical mode and the mode associated with thermophysical properties. This is exemplified by comparing results with a constant semi-local friction Reynolds number, namely cases AM3R7 and PM1R3 with $R e_{\tau, c}^{*} \approx 200$. Some particular features are nonetheless observed with PP11 flows, notably for the highest value of the Mach number, $M_{B}=3$. Furthermore the length scales of near-wall structures have been quantified using premultiplied one-dimensional spectra. The streamwise and spanwise non-dimensional length of the structures are found to be universal with respect to constant property when semi-local coordinates are used. The density field near the wall looks however different because the streaks correspond directly to high- and low-density fluid region for a perfect gas whereas instantaneous density follows pressure fluctuations in PP11 flows, since density fluctuations are now correlated with pressure ones. Additionally, joint-pdfs of streamwise and wall-normal velocity fluctuations reveal that the generation of Reynolds stresses follows the classical picture of wall turbulence when variable properties are included in the semi-local scaling. A slight reduction of ejection events is observed for PP11 flows.

Although non-classical dense-gas effects (like, e.g. unconventional shocklets) are not present in turbulent channel flows, where the turbulent Mach number remains small even for the highest values of $M_{B}$, the present results allow for the first time to shed some light on wall-bounded dense-gas turbulence, for which no experimental data are available, and open the way to the development of numerical models for the prediction of more complex configurations, such as turbine cascades. In particular, information obtained about the appropriate scalings, the behaviour of thermophysical properties, and grid resolution requirements may serve as a reference for justifying some assumptions in turbulence modelling for Reynolds-averaged or large eddy simulations. A priori analyses of turbulence models applied to compressible dense-gas flows are planned as future research.

Additional investigation is ongoing to check if the present conclusions still hold when investigating different operating thermodynamic conditions, retaining the same bulk Mach and Reynolds numbers, or by using other dense-gas fluids, such as refrigerants and siloxanes, for which more accurate and complex equations of state exist. Lastly, the study of turbulent boundary layers and the interactions with shock waves would be a further step to investigate the effects of higher Mach numbers and the modification of shock structure in presence of a dense gas.

\section{Acknowledgement}

This work was granted access to the HPC resources of GENCI (Grand Equipement National de Calcul Intensif) under the allocation 7332.

\section{Appendix A. Transport properties for dense gases}

Transport properties are generally considered to be dependent only on temperature values. Nevertheless, when $1<T / T_{c r}<1.5$ and when $p>p_{c r}$ (where $(\cdot)_{c r}$ denotes 
critical quantities), pressure has a strong effect on viscosity and thermal conductivity. This effect is usually taken into account by applying correction factors in the formula used to estimate the dilute-gas properties (i.e. the values of viscosity and thermal conductivity in the ideal-gas limit). In the following, we describe the laws derived by Chung, Lee \& Starling (1984), Chung et al. (1988).

\section{A.1. Dynamic viscosity}

The Chapman-Enskog theory describes the integral relations for the transport properties when the interactions between colliding molecules are described by a potential energy function. In general terms, the first-order solution for the dilute gas viscosity can be written as:

$$
\mu_{0}=\frac{26.69(\mathcal{M} T)^{1 / 2}}{\sigma^{2} \Omega_{v}(T)}
$$

where $\mathcal{M}$ is the molecular weight, $T$ the temperature, $\Omega_{v}(T)$ the temperature dependence of the collision integral and $\sigma\left(v_{c r}\right)$ the collision diameter. In the derivation of (A 1), $\Omega_{v}$ is obtained as a function of a dimensionless temperature $T^{*}$, defined as (Poling et al. 2001):

$$
T^{*}=\frac{k_{B} T}{\epsilon}
$$

where $k_{B}$ is Boltzmann's constant $\left(k_{B}=1.38064852 \times 10^{-23} \mathrm{~J} \mathrm{~K}^{-1}\right)$ and $\epsilon$ is the potential energy parameter. Neufeld, Janzen \& Aziz (1972) proposed the following empirical equation:

$$
\Omega_{v}=\frac{A}{T^{* B}}+\frac{C}{\exp \left(D T^{*}\right)}+\frac{E}{\exp \left(F T^{*}\right)}+G T^{* B} \sin \left(S T^{* W}-H\right),
$$

with $A=1.16145, B=0.14874, C=0.52487, D=0.77320, E=2.16178, F=2.43787$ $G=-6.436 \times 10^{-4}, H=7.27371, S=18.0323$ and $W=-0.76830$.

The method of Chung et al. $(1984,1988)$ starts from (A 1) with $\epsilon / k_{B}=T_{c r} / 1.2593$ and $\sigma=0.809 v_{c r}^{1 / 3}$, where $T_{c r}$ and $v_{c r}$ are the critical temperature and volume, respectively. Furthermore, they multiply the right-hand side of (A 1) by a factor $F_{c}$ in order to account for molecular shapes and polarities of dilute gases. The final result for the dilute-gas viscosity $\mu_{0}$ reads:

$$
\mu_{0}=40.785 \frac{F_{c}(\mathcal{M} T)^{1 / 2}}{v_{c r}^{2 / 3} \Omega_{v}}
$$

with

$$
F_{c}=1-0.2756 \omega+0.059035 \xi_{r}^{4}+\varpi .
$$

In (A 5), $\omega$ is the acentric factor, $\xi_{r}$ is the reduced dipole moment, and $\varpi$ is an empirically determined association parameter to characterize the molecular structure effect of polyatomic molecules, the polar effect, and the hydrogen-bonding effect, respectively. Specifically, the reduced dipole moment $\xi_{r}$ reads:

$$
\xi_{r}=131.3 \frac{\xi}{\left(v_{c r} T_{c r}\right)^{1 / 2}},
$$




$\begin{array}{lcccc}i & a_{0} & a_{1} & a_{2} & a_{3} \\ 1 & 6.32402 & 50.4119 & -51.6801 & 1189.02 \\ 2 & 0.00012102 & -0.0011536 & -0.0062571 & 0.037283 \\ 3 & 5.28346 & 254.209 & -168.481 & 3898.27 \\ 4 & 6.62263 & 38.0957 & -8.46414 & 31.4178 \\ 5 & 19.7454 & 7.63034 & -14.3544 & 31.5267 \\ 6 & -1.89992 & -12.5367 & 4.98529 & -18.1507 \\ 7 & 24.2745 & 3.44945 & -11.2913 & 69.3466 \\ 8 & 0.79716 & 1.11764 & 0.012348 & -4.11661 \\ 9 & -0.23816 & 0.067695 & -0.81630 & 4.02528 \\ 10 & 0.068629 & 0.34793 & 0.59256 & -0.72663\end{array}$

TABLE 7. Parameters for evaluating the functions $A_{i}$ of (A 12) for the computation of the dense-gas viscosity.

whereas the association parameter $\varpi$ is (Chung et al. 1988):

$$
\varpi=0.0682+4.704 \frac{\text { [number of }-\mathrm{OH} \text { groups }]}{\mathcal{M}} .
$$

For non-polar fluids, the last two terms of (A 5) become zero.

In order to take into account dense-gas effects, the dense-gas viscosity $\mu$ writes:

$$
\mu=\mu_{k}+\mu_{p}
$$

being $\mu_{k}$ a modified dilute-gas viscosity and $\mu_{p}$ a correction term; specifically,

$$
\begin{gathered}
\mu_{k}=\mu_{0}\left[1 / G+A_{6} Y\right] \\
\mu_{p}=36.44 \times 10^{-6} \frac{\left(\mathcal{M} T_{c r}\right)^{1 / 2}}{v_{c r}^{2 / 3}} A_{7} Y^{2} G \exp \left[A_{8}+A_{9}\left(T^{*}\right)^{-1}+A_{10}\left(T^{*}\right)^{-2}\right] .
\end{gathered}
$$

In (A 9) and (A 10), $Y=\rho v_{c r} / 6$ is a density-dependent term, $G$ is a nonlinear function written as

$$
G=\frac{A_{1}\left[1-\exp \left(-E_{4} Y\right)\right] / Y+A_{2} X \exp \left(A_{5} Y+A_{3} X\right)}{A_{1} A_{4}+A_{2}+A_{3}}, \quad X=\frac{1-0.5 Y}{(1-Y)^{3}}, \quad(\text { A } 11 a, b)
$$

and parameters $A_{1}-A_{10}$ are linear functions of $\omega, \xi_{r}$ and $\varpi$ :

$$
A_{i}=a_{0}(i)+a_{1}(i) \omega+a_{2}(i) \xi_{r}^{4}+a_{3}(i) \varpi, \quad i \in\{1, \ldots, 10\} .
$$

Values for the coefficients $a_{0}, a_{1}, a_{2}$ and $a_{3}$ are given in table 7 . One should note that at very low densities, $Y$ approaches zero, $X$ and $G$ approach unity and the dense-gas term becomes negligible, with (A 10) reducing to (A 1).

\section{A.2. Thermal conductivity}

The dense-gas thermal conductivity is derived, in a similar way, as the sum of a dilutegas component and a correction term. The dilute-gas component is written as

$$
\kappa_{0}=7.452 \frac{\mu_{0} \Psi}{\mathcal{M}},
$$


with

$$
\Psi=1+\alpha \frac{0.215+0.28288 \alpha-1.061 \beta+0.26665 \delta}{0.6366+\beta \delta+1.061 \alpha \beta} .
$$

Here, $\beta=0.7682-0.7109 \omega+1.3168 \omega^{2}$ is a fluid-dependent parameter, whereas $\alpha=c_{v, \infty}(T) / R-3 / 2$ and $\delta=2+10.5\left(T / T_{c r}\right)^{2}$ are temperature-dependent functions $\left(c_{v, \infty}(T)\right.$ being the ideal-gas heat capacity at constant volume and $R$ the gas constant). The term $\beta$ is an empirical correlation for the contribution of the internal degrees of freedom of the molecule, and is applied only for non-polar materials. For polar substances $\beta$ is specific for each compound, and Chung et al. (1984) list values for a few materials. If the compound is polar and $\beta$ is not available, a default value of $\beta=0.758$ is used. The parameter $\delta$ represents instead the number of collisions required to interchange a quantum of rotational energy with a quantum translational energy.

The dense-gas thermal conductivity $\kappa$ is computed by using the same approach as for viscosity:

$$
\kappa=\kappa_{k}+\kappa_{p},
$$

with

$$
\kappa_{k}=\kappa_{0}\left[1 / H+B_{6} Y\right] ; \quad \kappa_{p}=3.039 \times 10^{-4} \sqrt{\frac{T}{\mathcal{M}}} \frac{1}{v_{c r}^{2 / 3}} B_{7} Y^{2} H .
$$

Again, one has

$$
\begin{gathered}
Y=\frac{\rho v_{c r}}{6} ; \\
H=\frac{B_{1}\left[1-\exp \left(-B_{4} Y\right)\right] / Y+B_{2} X \exp \left(B_{5} Y+B_{3} X\right)}{B_{1} B_{4}+B_{2}+B_{3}} ; \quad X=\frac{1-0.5 Y}{(1-Y)^{3}} ;(\mathrm{A} 18 a, b) \\
B_{i}=b_{0}(i)+b_{1}(i) \omega+b_{2}(i) \xi_{r}^{4}+b_{3}(i) \varpi, \quad i \in\{1, \ldots, 7\}, \quad \text { (A 17) }
\end{gathered}
$$

with values of $b_{0}, b_{1}, b_{2}$ and $b_{3}$ given in table 8 .

\section{Appendix B. Effect of bulk viscosity}

Almost no results are available about the role of the volume or bulk viscosity for turbulent wall-bounded flows of complex molecular fluids, such as the dense gas PP11. Cramer (2012) showed that some fluids can exhibit bulk viscosities which are hundreds of times larger than their shear viscosities. That is why the assumption used in the present study of a Stokesian fluid, i.e. with a negligible bulk viscosity, is tested for one case, namely PM1R3 $\left(M_{B}=1.5, R e_{B}=3000\right)$ keeping exactly the same parameters (see table 3 ) but with $\mu_{b}=500 \mu$, as defined in $\S 2.1$. We selected a relatively low Reynolds number for cost reasons since the high values of the viscosity induce a further time step restriction for the explicit time marching scheme due to the limitation on the mesh Fourier number. Consequently, the only numerical parameter which is modified is the time step, which is divided approximatively by a factor of 


$\begin{array}{ccccc}i & b_{0} & b_{1} & b_{2} & b_{3} \\ 1 & 2.41657 & 0.74824 & -0.91858 & 121.721 \\ 2 & -0.50924 & -1.50936 & -49.9912 & 69.9834 \\ 3 & 6.61069 & 5.62073 & 64.7599 & 27.0389 \\ 4 & 14.5425 & -8.91387 & -5.63794 & 74.3435 \\ 5 & 0.79274 & 0.82019 & -0.69369 & 6.31734 \\ 6 & -5.86340 & 12.8005 & 9.58926 & -65.5292 \\ 7 & 81.1710 & 114.158 & -60.8410 & 466.775\end{array}$

TABLE 8. Parameters for evaluating the functions $B_{i}$ of (A 19) for the computation of the dense-gas thermal conductivity.

(a)

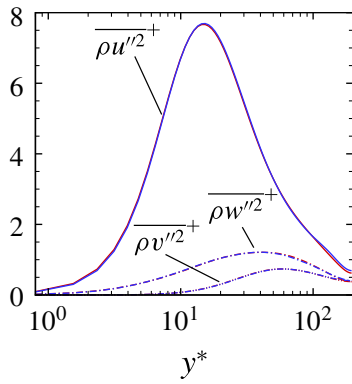

(b)

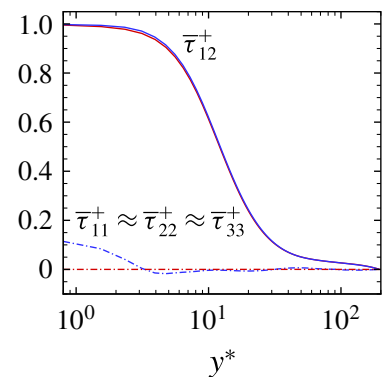

(c)

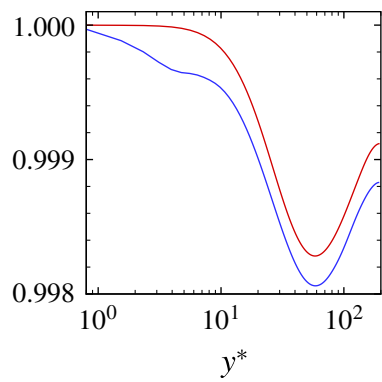

FIGURE 24. (Colour online) Effect of bulk viscosity. (a) Reynolds stresses, $(b)$ viscous stresses, $(c) \bar{p} / \bar{p}_{w}$ for case PM1R3. — (red): no bulk viscosity — (blue): $\mu_{b} / \mu=500$.

fifteen, implying a large number of iterations to achieve convergence of the statistics. The results are presented in figure 24. Second-order statistics such as the Reynolds stresses (figure 24a) are almost unaffected compared to the simulation with zero bulk viscosity, and the semi-local friction Reynolds number $R e_{\tau}^{*}$ changes approximatively $0.4 \%$, proving that the effects of bulk viscosity are negligible. Cramer \& Bahmani (2014) have recently examined large-Reynolds number flows of fluids having large bulk viscosities. By using the laminar boundary layer equations, they noticed some variations of the thermodynamic pressure across the boundary layer. The distributions of the viscous stresses and of the mean pressure across the channel are investigated in figure $24(b, c)$, respectively. In the case without bulk viscosity (red lines), the diagonal terms of the viscous stress tensor ${\overline{\tau_{i i}}}^{+}$are almost zero, and since in the viscous sublayer $\bar{\rho} \widetilde{v^{\prime \prime} v^{\prime \prime}} \approx 0$, the pressure remains constant according to (4.4). This is not the case when considering a high value of bulk viscosity, being $\bar{\tau}_{i i}+$ terms as high as $10 \%$ of the wall shear stress close to the walls, causing a decrease of the pressure in the sublayer. Nevertheless, $\bar{\tau}_{12}+$ is almost unchanged with and without bulk viscosity. Additionally, variations of thermodynamic quantities, observed only in the viscous sublayer, are less than $0.5 \%$ and are, therefore, negligible. These results confirm the use of Stokes' hypothesis in the present DNS study. 
Case

Subsonic (Kim et al. 1987)

Supersonic (Coleman et al. 1995)

$$
\begin{array}{cccc}
R e_{B} & M_{B} & L_{x} \times L_{y} \times L_{z} & N_{x} \times N_{y} \times N_{z} \\
2795 & 0.3 & 4 \pi h \times 2 h \times 2 \pi h & 192 \times 180 \times 160 \\
3000 & 1.5 & 8 \pi h \times 2 h \times 2 \pi h & 512 \times 256 \times 256
\end{array}
$$

TABLE 9. Parameters used for the DNS of the selected test cases.

$\begin{array}{lcccccccccr}\text { Case } & R e_{\tau_{w}} & R e_{\tau, c}^{*} & \Delta x^{+} & \Delta z^{+} & \Delta y_{w}^{+} & \Delta y_{c}^{+} & -B_{q} & \bar{\rho}_{w} / \rho_{B} & \bar{\rho}_{c} / \rho_{B} & \bar{T}_{c} / T_{w} \\ \text { Subsonic } & 181 & 178 & 11.9 & 7.2 & 0.81 & 4.1 & -0.002 & 1.01 & 0.99 & 1.01 \\ \text { Supersonic } & 219 & 147 & 10.7 & 5.4 & 0.78 & 3.2 & -0.049 & 1.36 & 0.98 & 1.39\end{array}$

TABLE 10. Results of the DNS computations.

(a)

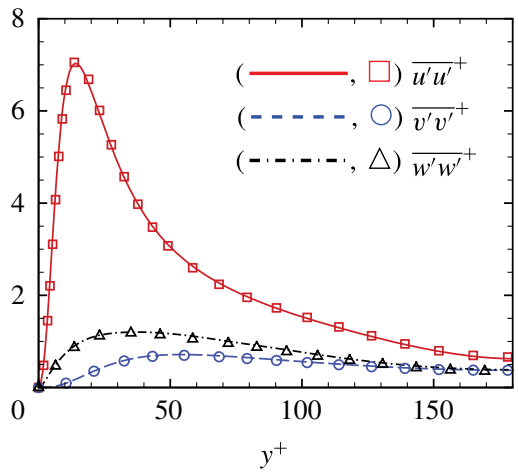

(b)

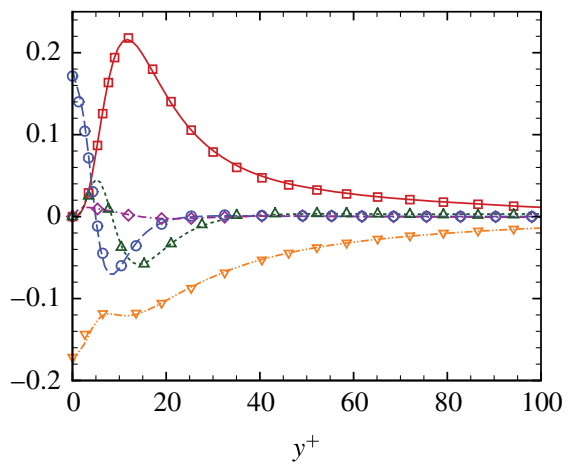

FIGURE 25. (Colour online) Validations for the subsonic channel flow case. Lines, present simulation; symbols, data from Kim et al. (1987). (a) R.m.s. velocity fluctuations; (b) turbulent kinetic energy budget. In $(b),(-, \square$ red), production; $(\cdots \cdots \cdots \cdot . \cdot \nabla$ orange) dissipation; $(\ldots \ldots \ldots, \Delta$ green) turbulent diffusion; $(-. . ., \bigcirc$ blue) viscous diffusion; $(\cdot \cdots . . . . . ., \diamond$ violet $)$ turbulent pressure diffusion.

\section{Appendix C. Validations}

In order to validate the code and the numerical strategy, two reference test cases have been considered, namely the incompressible channel flow of Kim et al. (1987) and supersonic isothermal channel flow of Coleman et al. (1995). The parameters of the DNS are summarized in table 9. Note that a low subsonic Mach number, $M_{B}=0.3$, has been chosen for the comparison of our compressible solver with the incompressible case. Table 10 shows the main results of our simulations. Figure 25 shows the profiles of r.m.s. velocity fluctuations and the evolution of the turbulent kinetic energy budgets compared to the data from Kim et al. (1987). Figure 26 shows similar comparison for the first- and second-order statistics for the supersonic case. A very good agreement with the reference results is obtained for both cases.

\section{Appendix D. Grid convergence study}

A study of the sensitivity to the grid resolution has been performed for the case $M_{B}=1.5, R e_{B}=3000$ with both air and PP11 fluids. The resolutions obtained for 
(a)

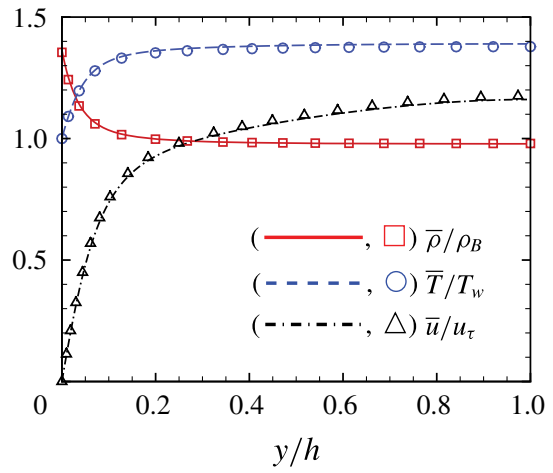

(b)

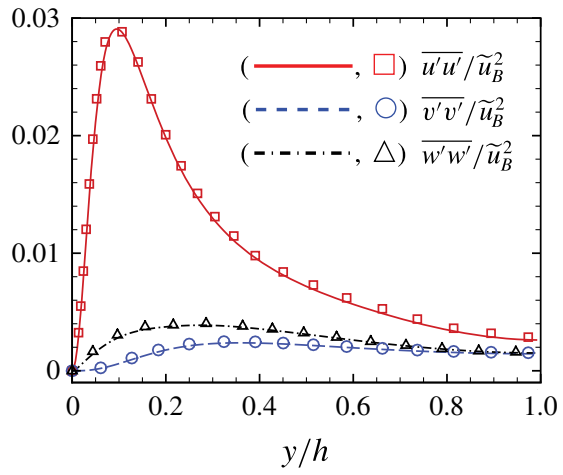

FIGURE 26. (Colour online) Validations for the supersonic channel flow case. Lines, present simulation; symbols, data from Coleman et al. (1995). (a) Profiles of mean quantities; (b) r.m.s. velocity fluctuations.

\begin{tabular}{|c|c|c|c|c|c|c|c|c|c|c|c|c|c|}
\hline \multirow[b]{2}{*}{ Line } & \multirow[b]{2}{*}{ Grid } & \multicolumn{6}{|c|}{ Air } & \multicolumn{6}{|c|}{ PP11 } \\
\hline & & $\Delta x^{*}$ & $\Delta y_{c}^{*}$ & $\Delta z^{*}$ & $\frac{\Delta x}{\eta}$ & $\frac{\Delta y_{c}}{\eta}$ & $\frac{\Delta z}{\eta}$ & $\Delta x^{*}$ & $\Delta y_{c}^{*}$ & $\Delta z^{*}$ & $\frac{\Delta x}{\eta}$ & $\frac{\Delta y_{c}}{\eta}$ & $\frac{\Delta z}{\eta}$ \\
\hline (orange) & $256 \times 128 \times 128$ & 14.6 & 6.4 & 7.3 & 13.8 & 1.8 & 6.9 & 19.4 & 8.0 & 9.8 & 12.4 & 2.1 & 6.2 \\
\hline (black) & $256 \times 256 \times 256$ & 14.5 & 2.2 & 3.6 & 13.7 & 0.7 & 3.4 & 19.3 & 2.3 & 4.8 & 12.2 & 0.7 & 3.0 \\
\hline (blue) & $512 \times 128 \times 256$ & 7.2 & 6.4 & 3.6 & 6.8 & 1.8 & 3.4 & 9.7 & 8.0 & 4.8 & 6.1 & 2.1 & 3.0 \\
\hline (green) & $512 \times 256 \times 128$ & 7.2 & 2.2 & 7.3 & 6.9 & 0.7 & 6.9 & 9.7 & 2.3 & 9.8 & 6.1 & 0.7 & 6.2 \\
\hline & $512 \times 256 \times 256$ & 7.2 & 2.2 & 3.6 & 6.8 & 0.7 & 3.4 & 9.7 & 2.3 & 4.8 & 6.1 & 0.7 & 3.0 \\
\hline 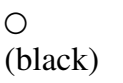 & $1024 \times 256 \times 512$ & 3.6 & 2.2 & 1.8 & 3.4 & 0.7 & 1.7 & 4.9 & 2.3 & 2.4 & 3.0 & 0.7 & 1.5 \\
\hline
\end{tabular}

TABLE 11. Grid resolution in semi-local units and normalized by the Kolmogorov length scale.

different grid levels are given in table 11 in semi-local units and normalized by the local Kolmogorov length scale. Figure 27 presents the results for the three components of the Reynolds stresses. At a first glance, we observe that the convergence by refining the grid is faster for air case. This is consistent with the fact that the dense-gas effects yield higher values of the centreline Reynolds number (figure 6). As a consequence, the values of cell sizes expressed in the semi-local coordinates are slightly higher for PP11 cases on a given grid. Nevertheless, the grid convergence analysis shows that the criteria used to select the grid sizes for our study do ensure a clear convergence, so that the results are essentially grid-independent. 
(a)

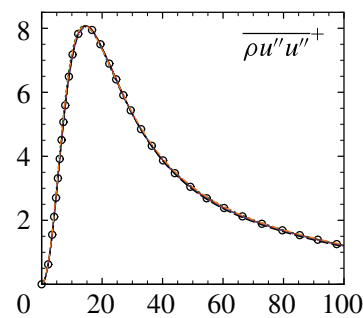

$(d)$

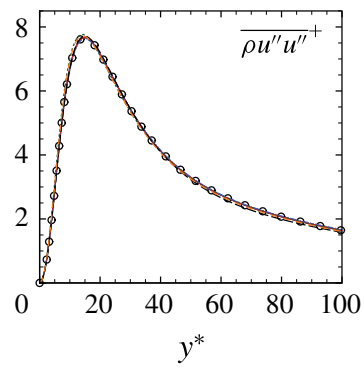

(b)

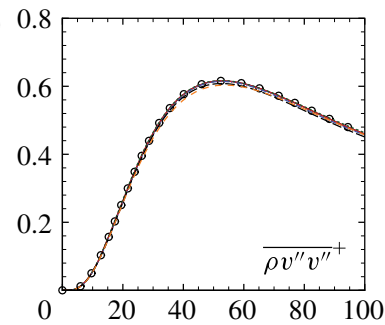

(e)

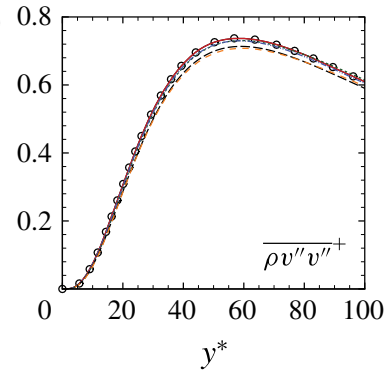

(c)
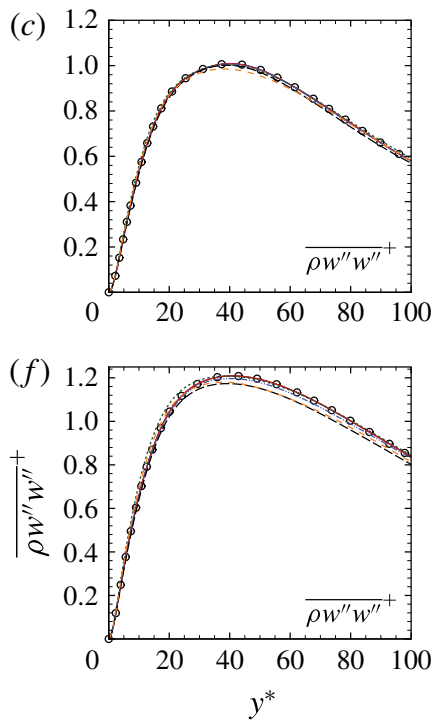

FIGURE 27. (Colour online) Grid convergence study for air $(a-c)$ and PP11 $(d-f)$ for case $M_{B}=1.5, \operatorname{Re}_{B}=3000$.

\section{REFERENCES}

Anderson, W. K. 1991 Numerical study on using sulfur hexafluoride as a wind tunnel test gas. AIAA J. 29 (12), 2179-2180.

Aubard, G., Gloerfelt, X. \& Robinet, J.-C. 2013 Large-eddy simulation of broadband unsteadiness in a shock/boundary-layer interaction. AIAA J. 51 (10), 2395-2409.

BAE, J. H., Yoo, J. Y. \& CHOI, H. 2005 Direct numerical simulation of turbulent supercritical flows with heat transfer. Phys. Fluids 17 (10), 105104.

Bogey, C. \& Bailly, C. 2004 A family of low dispersive and low dissipative explicit schemes for flow and noise computations. J. Comput. Phys. 194 (1), 194-214.

Bogey, C. \& BAilly, C. 2009 Turbulence and energy budget in a self-preserving round jet: direct evaluation using large eddy simulation. J. Fluid Mech. 627, 129-160.

Bogey, C., De Cacqueray, N. \& Bailly, C. 2009 A shock-capturing methodology based on adaptative spatial filtering for high-order non-linear computations. J. Comput. Phys. 228 (5), $1447-1465$.

Bogey, C., Marsden, O. \& Bailly, C. 2012 Influence of initial turbulence level on the flow and sound fields of a subsonic jet at a diameter-based Reynolds number of 105. J. Fluid Mech. 701, 352-385.

Bradshaw, P. 1977 Compressible turbulent shear layers. Annu. Rev. Fluid Mech. 9 (1), 33-52.

Brown, B. P. \& Argrow, B. M. 2000 Application of Bethe-Zel'dovich-Thompson fluids in organic Rankine cycle engines. J. Propul. Power 16 (6), 1118-1124.

Brun, C., Boiarciuc, M. P., Hakerborn, M. \& Comte, P. 2008 Large eddy simulation of compressible channel flow - arguments in favour of universality of compressible turbulent wall bounded flows. Theor. Comput. Fluid Dyn. 22, 189-212.

Bufi, E. A. \& Cinnella, P. 2015 Efficient uncertainty quantification of turbulent flows through supersonic ORC nozzle blades. Energy Procedia 82, 186-193.

Chang, P. A. III, Piomelli, U. \& Blake, W. K. 1999 Relationship between wall pressure and velocity-field sources. Phys. Fluids 11 (11), 3434-3448. 
Chernyshenko, S. I. \& BAig, M. F. 2005 The mechanism of streak formation in near-wall turbulence. J. Fluid Mech. 544 (1), 99-131.

Chu, B.-T. \& Kovasznay, L. S. G. 1958 Non-linear interactions in a viscous heat-conducting compressible gaz. J. Fluid Mech. 3, 494-514.

Chung, T. H., Ajlan, M., Lee, L. L. \& Starling, K. E. 1988 Generalized multiparameter correlation for nonpolar and polar fluid transport properties. Indust. Engng Chem. Res. 27 (4), 671-679.

Chung, T. H., Lee, L. L. \& Starling, K. E. 1984 Applications of kinetic gas theories and multiparameter correlation for prediction of dilute gas viscosity and thermal conductivity. Indust. Engng Chem. Fundamentals 23 (1), 8-13.

Cinnella, P. \& Congedo, P. M. 2007 Inviscid and viscous aerodynamics of dense gases. J. Fluid Mech. 580, 179-217.

Coleman, G. N., Kim, J. \& Moser, R. D. 1995 A numerical study of turbulent supersonic isothermal-wall channel flow. J. Fluid Mech. 305, 159-183.

Congedo, P. M., Corre, C. \& Cinnella, P. 2011 Numerical investigation of dense-gas effects in turbomachinery. Comput. Fluids 49 (1), 290-301.

Cramer, M. S. 1989 Negative nonlinearity in selected fluorocarbons. Phys. Fluids 1 (11), 1894-1897.

Cramer, M. S. \& BAhmani, F. 2014 Effect of large bulk viscosity on large-Reynolds-number flows. J. Fluid Mech. 751, 142-163.

Cramer, M. S. \& Kluwick, A. 1984 On the propagation of waves exhibiting both positive and negative nonlinearity. J. Fluid Mech. 142 (1), 9-37.

CRAMER, M. S. \& PARK, S. 1999 On the suppression of shock-induced separation in Bethe-Zel'dovich-Thompson fluids. J. Fluid Mech. 393, 1-21.

Cramer, M. S. \& TArkenton, G. M. 1992 Transonic flows of Bethe-Zel'dovich-Thompson fluids. J. Fluid Mech. 240, 197-228.

Cramer, M. S. 2012 Numerical estimates for the bulk viscosity of ideal gases. Phys. Fluids 24 (6), 066102.

DEAN, R. B. 1978 Reynolds number dependence of skin friction and other bulk flow variables in two-dimensional rectangular duct flow. Trans. ASME J. Fluids Engng 100 (2), 215-223.

Donzis, D. A. \& Jagannathan, S. 2013 Fluctuations of thermodynamic variables in stationary compressible turbulence. J. Fluid Mech. 733, 221-244.

Duan, L., BeEkMAN, I. \& Martin, M. P. 2010 Direct numerical simulation of hypersonic turbulent boundary layers. Part 2. Effect of wall temperature. J. Fluid Mech. 655, 419-445.

Foysi, H., SARKAR, S. \& FrIEdRICH, R. 2004 Compressibility effects and turbulence scalings in supersonic channel flow. J. Fluid Mech. 509, 207-216.

Fukagata, K., Iwamoto, K. \& Kasagi, N. 2002 Contribution of Reynolds stress distribution to the skin friction in wall-bounded flows. Phys. Fluids 14 (11), L73-L76.

Gerolymos, G. A., SÉnéchal, D. \& VAllet, I. 2010 Performance of very-high-order upwind schemes for DNS of compressible wall-turbulence. Intl J. Numer. Meth. Fluids 63 (7), 769-810.

Gerolymos, G. A. \& VAllet, I. 2014 Pressure, density, temperature and entropy fluctuations in compressible turbulent plane channel flow. J. Fluid Mech. 757, 701-746.

Gloerfelt, X. \& Berland, J. 2013 Turbulent boundary-layer noise: direct radiation at Mach number 0.5. J. Fluid Mech. 723, 318-351.

Gomez, T., Flutet, V. \& Sagaut, P. 2009 Contribution of Reynolds stress distribution to the skin friction in compressible turbulent channel flows. Phys. Rev. E 79 (3), 035301.

Guardone, A. \& ARgrow, B. M. 2005 Nonclassical gasdynamic region of selected fluorocarbons. Phys. Fluids 17 (11), 116102.

Guarini, S. E., Moser, R. D., Shariff, K. \& Wray, A. 2000 Direct numerical simulation of a supersonic turbulent boundary layer at Mach 2.5. J. Fluid Mech. 414, 1-33.

Huang, P. G., Bradshaw, P. \& Coakley, T. J. 1993 Skin friction and velocity profile family for compressible turbulent boundary layers. AIAA J. 31 (9), 1600-1604.

Huang, P. G., Coleman, G. N. \& Bradshaw, P. 1995 Compressible turbulent channel flows: DNS results and modelling. J. Fluid Mech. 305, 185-218. 
Incropera, F. P. \& DeWitt, D. P. 2007 Fundamentals of Heat and Mass Transfer, 6th edn. Wiley.

KIM, J. 1989 On the structure of pressure fluctuations in simulated turbulent channel flow. J. Fluid Mech. 205, 421-451.

Kim, J., Moin, P. \& Moser, R. 1987 Turbulence statistics in fully developed channel flow at low Reynolds number. J. Fluid Mech. 177, 133-166.

Lagha, M., Kim, J., Eldredge, J. D. \& Zhong, X. 2011 A numerical study of compressible turbulent boundary layers. Phys. Fluids 23 (1), 015106.

LAUfER, J. 1969 Thoughts on compressible turbulent boundary layers. NASA S.P. 216.

Lechner, R., Sesterhenn, J. \& Friedrich, R. 2001 Turbulent supersonic channel flow. J. Turbul. 2 (1), 001-001.

LeE, J., Jung, S. Y., Sung, H. J. \& ZAKI, T. A. 2013 Effect of wall heating on turbulent boundary layers with temperature-dependent viscosity. J. Fluid Mech. 726, 196-225.

Lee, M. \& Moser, R. D. 2015 Direct numerical simulation of turbulent channel flow up to $R e_{\tau} \approx$ 5200. J. Fluid Mech. 774, 395-415.

Li, X., Hashimoto, K., Tominaga, Y., Tanahashi, M. \& Miyauchi, T. 2008 Numerical study of heat transfer mechanism in turbulent supercritical $\mathrm{CO}_{2}$ channel flow. J. Therm. Sci. Technol. 3 (1), 112-123.

Martin, J. J. \& Hou, Y. C. 1955 Development of an equation of state for gases. AIChE J. 1 (2), $142-151$.

Mathiussen, T., Gallo, M., Casati, E., Nannan, N. R., Zamfirescu, C., Guardone, A. \& Colonna, P. 2015 The flexible asymmetric shock tube (FAST): a Ludwieg tube facility for wave propagation measurements in high-temperature vapours of organic fluids. Exp. Fluids $\mathbf{5 6}$ (10), 1-12.

Modesti, D. \& Pirozzoli, S. 2016 Reynolds and Mach number effects in compressible turbulent channel flow. Intl J. Heat Fluid Flow 59, 33-49.

Monaco, J. F., Cramer, M. S. \& Watson, L. T. 1997 Supersonic flows of dense gases in cascade configurations. J. Fluid Mech. 330, 31-59.

MoneghAN, R. J. 1953 A review and assessment of various formulae for tubulent skin friction in compressible flow. Tech. Rep. Aeronautical Research Council. Current Paper 142.

Morinishi, Y., TAmano, S.\& NAKABAYAShi, K. 2004 Direct numerical simulation of compressible turbulent channel flow between adiabatic and isothermal walls. J. Fluid Mech. 502, 273-308.

Morkovin, M. V. 1961 Effect of compressibility on turbulent flows. In Mécanique de la Turbulence (ed. A. Favre), pp. 367-380. CNRS.

Moser, R., Kim, J. \& MAnsour, N. N. 1999 Direct numerical simulation of turbulent channel flow up to $r e_{\tau}=590$. Phys. Fluids 11, 943-945.

Neufeld, P. D., JAnZEN, A. R. \& AzIZ, R. A. 1972 Empirical equations to calculate 16 of the transport collision integrals $\Omega^{(l, s) *}$ for the Lennard-Jones (12-6) potential. J. Chem. Phys. 57 (3), 1100-1102.

Nicoud, F. \& PoInsot, T. 1999 DNS of a channel flow with variable properties. In Proceedings of First International Symposium on Turbulence and Shear Flow Phenomena, TSFP-1, Santa Barbara, USA, TSFP.

Patel, A., Peeters, J. W. R., Boersma, B. J. \& Pecnik, R. 2015 Semi-local scaling and turbulence modulation in variable property turbulent channel flows. Phys. Fluids 27 (9), 095101.

Pirozzoli, S. \& Bernardini, M. 2011 Turbulence in supersonic boundary layers at moderate Reynolds number. J. Fluid Mech. 688, 120-168.

Pirozzoli, S., Grasso, F. \& GATSKi, T. B. 2004 Direct numerical simulation and analysis of a spatially evolving supersonic turbulent boundary layer at $M=2.25$. Phys. Fluids 16 (3), 530-545.

Poling, B. E., Prausnitz, J. M., O’Connell, J. P. \& Reid, R. C. 2001 The Properties of Gases and Liquids, vol. 5. McGraw-Hill.

Rubesin, M. W. 1990 Extra compressibility terms for Favre-averaged two-equation models of inhomogeneous turbulent flows. NASA Contractor Rep. 177556. 
SARKAR, S. 1995 The stabilizing effect of compressibility in turbulent shear flow. J. Fluid Mech. 282, $163-186$.

Sciacovelli, L. \& Cinnella, P. 2015 Numerical simulation of dense gas compressible homogeneous isotropic turbulence. In 15th European Turbulence Conference, EUROMECH/ETC15.

Sciacovelli, L., Cinnella, P., Content, C. \& Grasso, F. $2016 a$ Dense gas effects in inviscid homogeneous isotropic turbulence. J. Fluid Mech. 800 (1), 140-179.

Sciacovelli, L., Cinnella, P. \& Grasso, F. $2016 b$ Small-scale dynamics of dense gas compressible homogeneous isotropic turbulence. J. Fluid Mech. (submitted).

Sewall, E. A. \& TAFTI, D. K. 2008 A time-accurate variable property algorithm for calculating flows with large temperature variations. Comput. Fluids 37, 51-63.

Sieder, E. N. \& TATE, G. E. 1936 Heat transfer and pressure drop of liquids in tubes. Indust. Engng Chem. 28 (12), 1429-1435.

SpinA, E. F., SMits, A. J. \& Robinson, S. K. 1994 The physics of supersonic turbulent boundary layers. Annu. Rev. Fluid Mech. 26, 287-319.

Spinelli, A., Pini, M., Dossena, V., Gaetani, P. \& Casella, F. 2013 Design, simulation, and construction of a test rig for organic vapors. Trans. ASME J. Engng Gas Turbines Power 135 (4), 042304.

TAMANO, S. \& MoRINISHI, Y. 2006 Effect of different thermal wall boundary conditions on compressible turbulent channel flow at $M=1.5$. J. Fluid Mech. 548, 361-373.

Teitel, M. \& Antonia, R. A. 1993 Heat transfer in fully developed turbulent channel flow: comparison between experiment and direct numerical simulations. Intl J. Heat Mass Transfer 36 (6), 1701-1706.

Thompson, P. A. 1971 A fundamental derivative in gasdynamics. Phys. Fluids 14 (9), 1843-1849.

Trettel, A. \& LARSSON, J. 2016 Mean velocity scaling for compressible wall turbulence with heat transfer. Phys. Fluids 28 (2), 026102.

VAN DRIEST, E. R. 1951 Turbulent boundary layer in compressible fluids. J. Aero. Sci. 18 (3), $145-160$.

WALlaCE, J. M. 2016 Quadrant analysis in turbulence research: history and evolution. Annu. Rev. Fluid Mech. 48, 131-158.

WeI, L. \& Pollard, A. 2011 Interactions among pressure, density, vorticity and their gradients in compressible turbulent channel flows. J. Fluid Mech. 673, 1-18.

ZoNTA, F. 2013 Nusselt number and friction factor in thermally stratified turbulent channel flow under Non-Oberbeck-Boussinesq conditions. Intl J. Heat Fluid Flow 44, 489-494.

Zonta, F., Marchioli, C. \& Soldati, A. 2012 Modulation of turbulence in forced convection by temperature-dependent viscosity. J. Fluid Mech. 697, 150-174. 